\title{
INVESTIGATION OF CREEP AND FATIGUE IN HIGH TEMPERATURE POLYMER MATRIX COMPOSITES USING A MICROMECHANICAL APPROACH
}

\author{
by \\ Alireza Sayyidmousavi \\ Bachelor of Science in Mechanical Engineering, Mazandaran University, Iran, 2001 \\ Master of Science in Mechanical Engineering, Khajeh Nasir Toosi University of Technology, Iran, 2005 \\ Master of Science in Mechanical Engineering, Ryerson University, Canada, 2010 \\ A dissertation presented to Ryerson University \\ in partial fulfillment of the \\ requirements for the degree of \\ Doctor of Philosophy \\ in the Program of \\ Mechanical and Industrial Engineering
}

Toronto, Ontario, Canada, 2014

(C) Alireza Sayyidmousavi, 2014 


\begin{abstract}
AUTHOR'S DECLARATION
I hereby declare that I am the sole author of this thesis. This is a true copy of the thesis, including any required final revisions, as accepted by my examiners.

I authorize Ryerson University to lend this thesis to other institutions or individuals for the purpose of scholarly research.

I further authorize Ryerson University to reproduce this thesis by photocopying or by other means, in total or in part, at the request of other institutions or individuals for the purpose of scholarly research.

I understand that my thesis may be made electronically available to the public.
\end{abstract}




\title{
ABSTRACT \\ Investigation of Creep and Fatigue in High Temperature Polymer Matrix Composites Using a Micromechanical Approach
}

Doctor of Philosophy, 2014

\author{
Alireza Sayyidmousavi \\ Mechanical and Industrial Engineering \\ Ryerson University
}

Polymer matrix composites (PMC's) are widely used in critical aerospace structures due to their numerous advantageous mechanical properties. Recently, PMC's have been considered for high temperature applications where viscoelasticity arising from the time dependent nature of the polymer matrix becomes an important consideration. This inherent viscoelasticity can significantly influence deformation, strength and failure response of these materials under different loading modes and environmental factors. With a potentially large number of plies of different fiber directions and perhaps material properties, determining a fatigue failure criterion of any degree of generality through experiments only, may seem to be an unrealistic task. This difficult situation may be mitigated through the development of suitable theoretical micro or macro mechanical models that are founded on considering the fatigue failure of the constituting laminas. The micro-approach provides a detailed examination of the individual failure modes in each of the constituent materials i.e. fiber, matrix. In this work, a micromechanical approach is used to study the role of viscoelasticity on the fatigue behavior of polymer matrix composites. In particular, the study examines the interaction of fatigue and creep in polymer matrix composites. The matrix phase is modeled as a vicoelastic material using Schapery's single integral constitutive equation. Taking viscoelsticity into account allows the study of creep strain evolution during the fatigue loading. The fatigue failure criterion is expressed in terms of 
the fatigue failure functions of the constituent materials. The micromechanical model is also used to calculate these fatigue failure functions from the knowledge of the S-N diagrams of the composite material in longitudinal, transverse and shear loadings thus eliminating the need for any further experimentation. Unlike the previous works, the present study can distinguish between the strain evolution due to fatigue and creep. The results can clearly show the contribution made by the effect of viscoelasticity to the total strain evolution during the fatigue life of the specimen. Although the effect of viscoelsticity is found to increase with temperature, its contribution to strain development during fatigue is compromised by the shorter life of the specimen when compared to lower temperatures. 


\section{ACKNOWLEDGEMENTS}

I would like to express my most sincere gratitude to my supervisors Dr. Habiba Bougherara and Dr. Zouheir Fawaz for their continuous guidance, support, and for providing a great learning environment throughout my PhD studies.

I would also like to thank Dr. Ihab Elsawi and Dr. Hamid Ghaemi for their invaluable help and guidance in the course of my experiments.

Finally, I would like to thank my parents to whom I owe this work and every other achievement I have made in life. 
TABLE OF CONTENTS

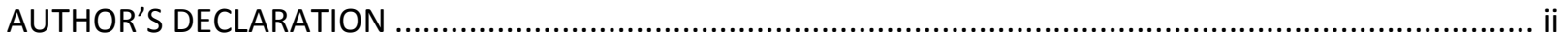

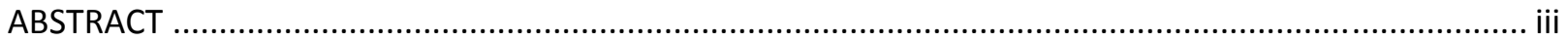

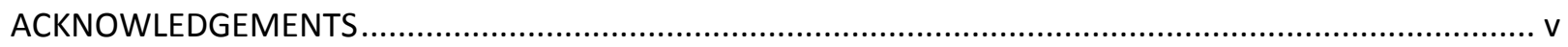

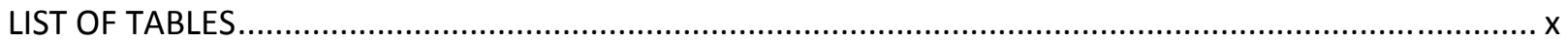

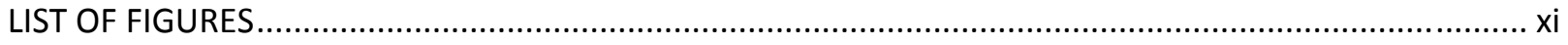

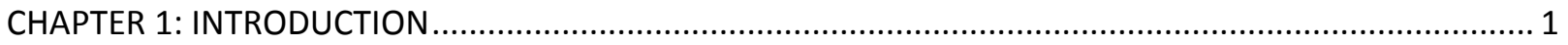

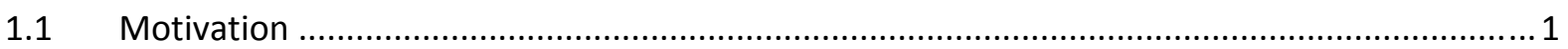

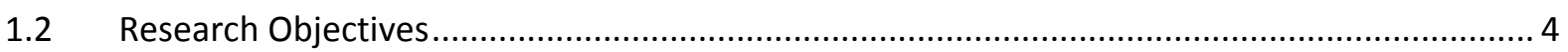

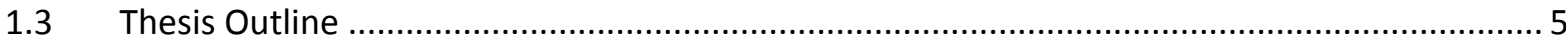

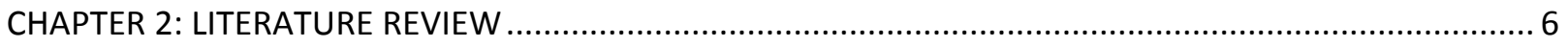

2.1 High Temperature Polymer Matrix Composites ................................................................... 6

2.2 Literature Review on the Creep Behavior of Fiber Reinforced Polymer Composites................. 8

2.2.1 Time dependent behavior of polymer matrix composite materials............................... 8

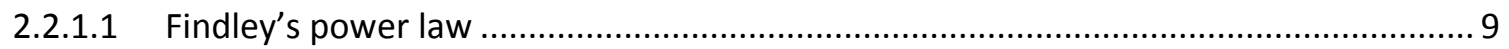

2.2.1.2 Boltzmann's superposition principle ….................................................................... 10

2.2.1.3 Schapery single integral model ................................................................................. 10

2.2.2 Creep failure of polymer matrix composites ............................................................... 12

2.2.2.1 Kinetic rate theory approach to creep failure ….................................................... 13

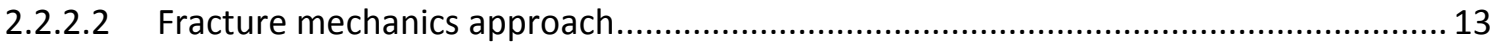

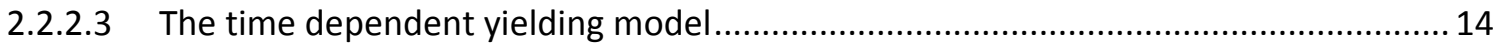

2.2.2.4 Energy based creep failure criteria ...................................................................... 15

2.3 Literature Review on the Fatigue of Fiber Reinforced Polymer Composites .......................... 16

2.3.1 Fatigue damage modeling of fiber reinforced composite materials ............................. 16

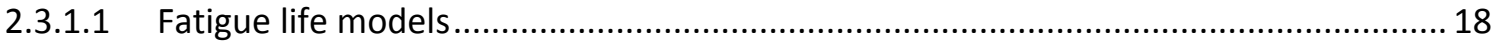

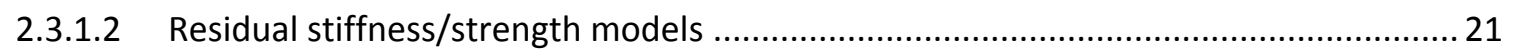

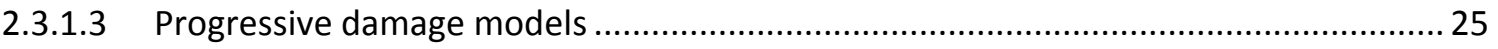

2.3.2 Effect of elevated temperature on the fatigue behavior of polymer matrix composites 30 


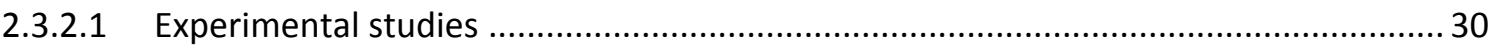

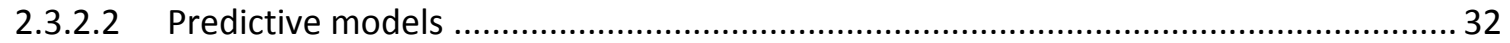

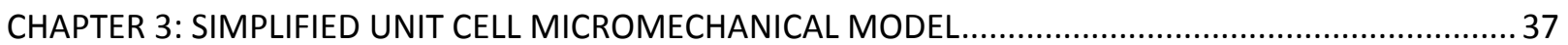

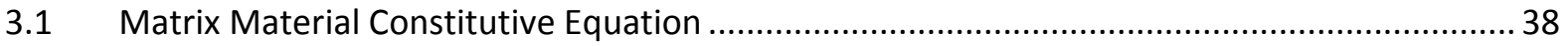

3.2 Assumptions of the Micromechanical Model ..................................................................... 40

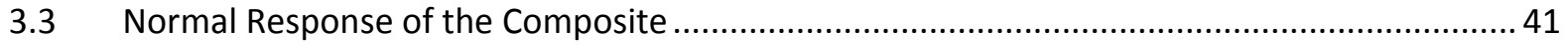

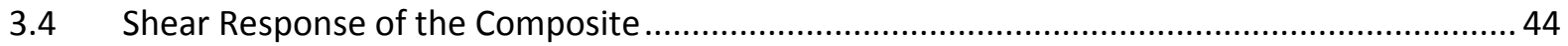

3.5 Micromechanical Analysis of the Composite Strength ........................................................ 45

CHAPTER 4: THE EFFECT OF TEMPERATURE ON THE VISCOELASTIC RESPONSE OF POLYMER MATRIX

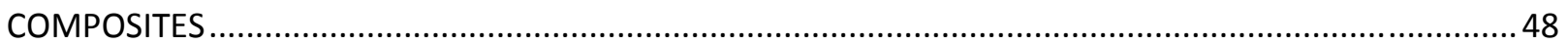

4.1 Effect of Temperature on the Modulus of the Polymer Matrix..............................................50

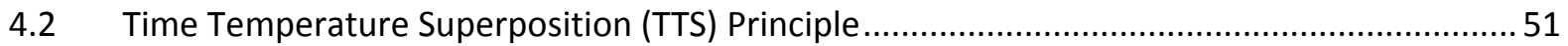

4.3 The Viscoelastic Response of Thermo-Stable PMR-15 …............................................... 52

4.4 Elastic Properties of Unidirectional T650-35/PMR-15 Composite as a Function of

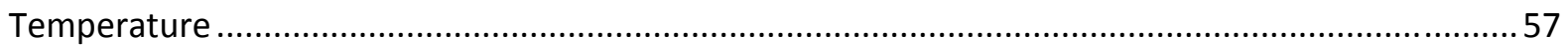

4.5 Thermomechanical Response under a Linearly Increasing Stress ........................................ 58

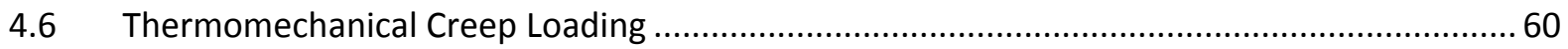

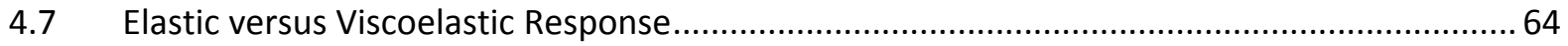

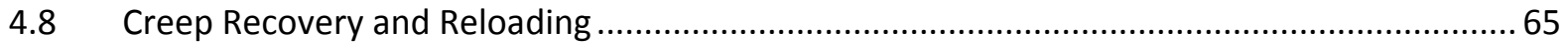

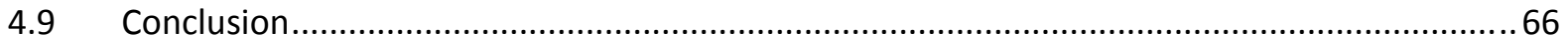

CHAPTER 5: A MICROMECHANICAL APPROACH FOR THE PREDICTION OF THE TIME-DEPENDENT FAILURE OF HIGH TEMPAERATURE POLYMER MATRIX COMPOSITES .................................................6

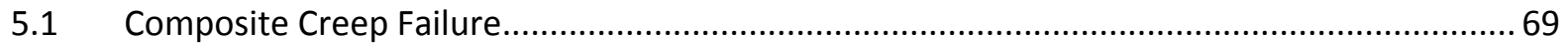


5.2 Long Term Behavior of T300/934 Composite Systems ....................................................... 70

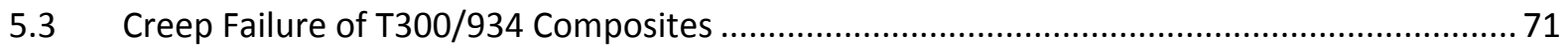

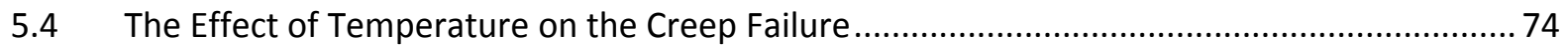

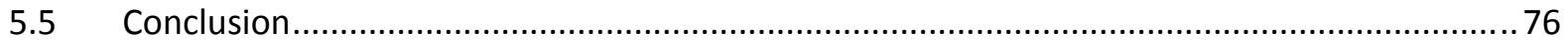

CHAPTER 6: THE ROLE OF VISCOELASTICITY ON THE FATIGUE OF ANGLE-PLY POLYMER MATRIX

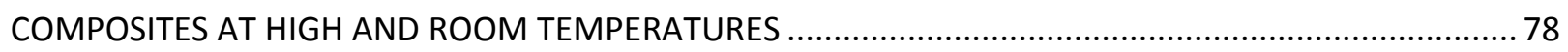

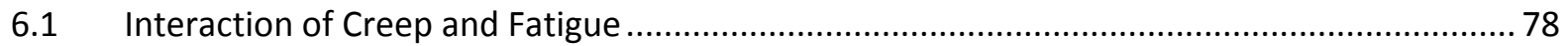

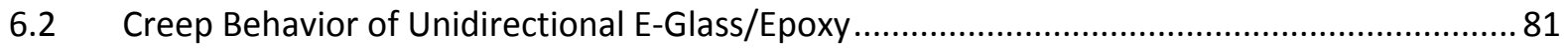

6.3 Fatigue behavior of unidirectional E-Glass/Epoxy ............................................................. 83

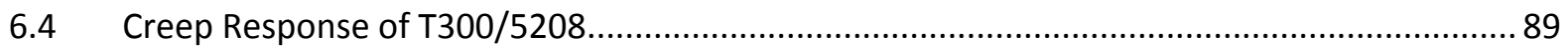

$6.5 \quad$ Fatigue Behavior of Angle-Ply T300/5208 ................................................................. 92

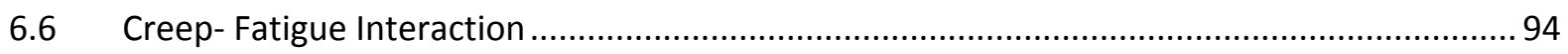

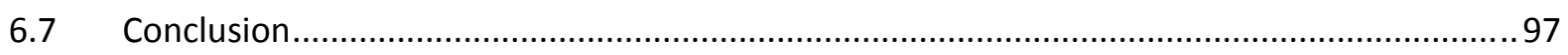

CHAPTER 7: A CASE-STUDY ON THE CREEP AND FATIGUE OF HIGH TEMPERATURE RP-46 POLYIMIDE

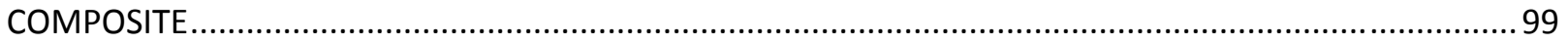

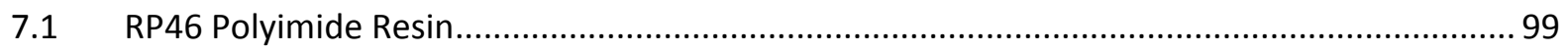

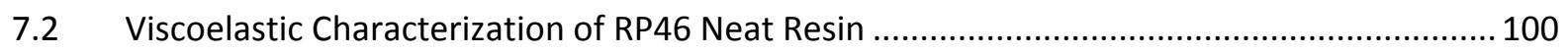

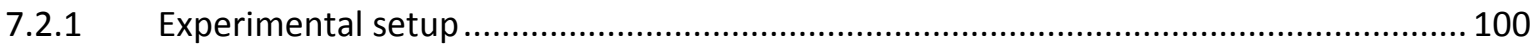

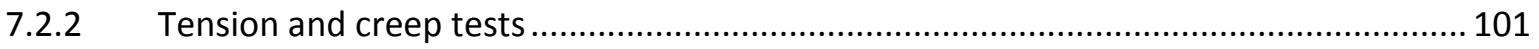

7.2.3 Prony Series representation of the creep data............................................................ 102

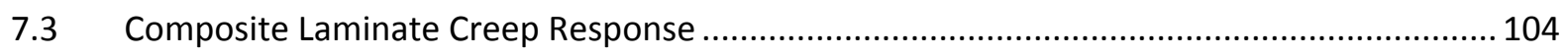

7.4 Effect of Viscoelasticity on the fatigue of Composite Laminate ......................................... 108

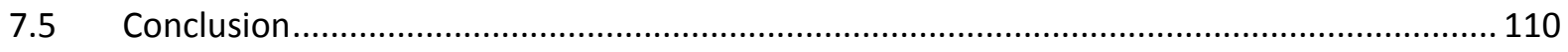




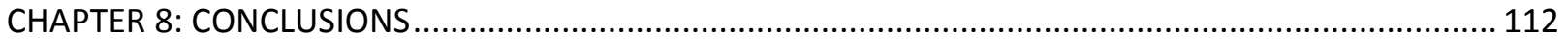

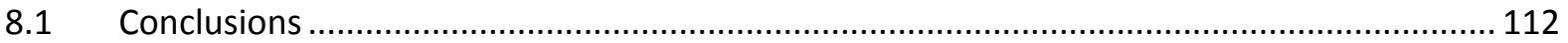

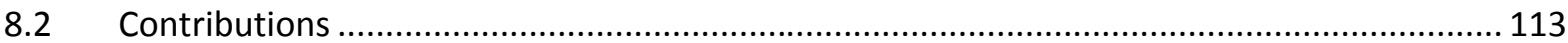

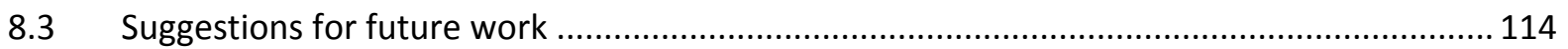

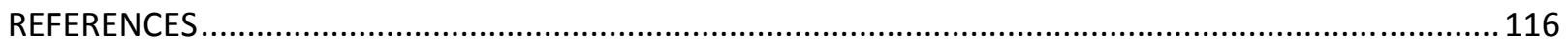




\section{LIST OF TABLES}

Table 4-1: Time-dependent properties of PMR-15 resin (Muliana and Sawant, 2009) ...........................55

Table 4-2: Elastic properties for T650-35 graphite fiber(Rupnowski et al., 2006) ................................ 58

Table 5-1: Glass and epoxy elastic material properties (Hiel, 1983) .................................................... 70

Table 6-1: Glass and epoxy elastic material properties, $V_{f}=0.476$ (Haj-Ali and Muliana, 2004a) ............82

Table 6-2: Prony series coefficients for the matrix material (Haj-Ali and Muliana, 2004a) .................... 82

Table 6-3: Graphite and epoxy elastic material properties (Haj-Ali and Muliana, 2004a) .....................89

Table 6-4: Prony Series coefficient for 5208 Epoxy matrix (Haj-Ali and Muliana, 2004a).......................90

Table 7-1: Prony Series coefficients for RP46 resin ....................................................................... 104 


\section{LIST OF FIGURES}

Figure 1-1: Boeing 787 material composition (Hawk, 2005) ................................................................... 1

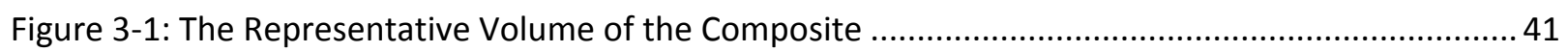

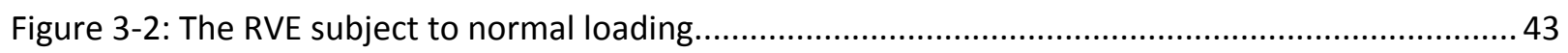

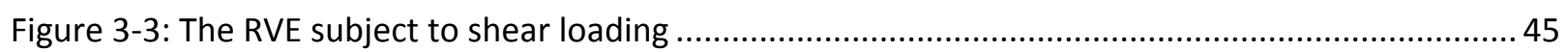

Figure 4-1: Variations in the Young modulus of a typical polymer with temperature (Brinson and

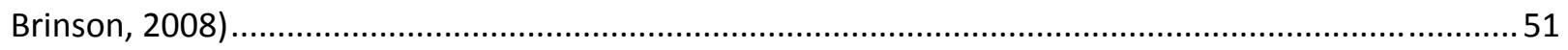

Figure 4-2: Schematic representation of how a master curve is generated ........................................5 52

Figure 4-3: compliance for PMR-15 as a thermorheologically simple material at different temperatures

Figure 4-4: Creep compliance for PMR-15 as a thermorheologically complex material at different

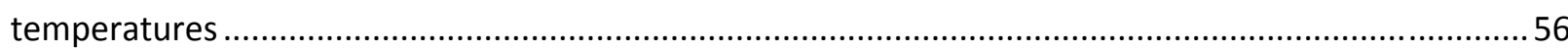

Figure 4-5: The elastic properties of unidirectional T650-35/PMR-15 composite: a) Axial modulus b)

Transverse modulus $\quad$ c) Shear modulus d) Longitudinal Poisson's ratio........................................... 58

Figure 4-6: Transverse stress vs. strain at different temperatures for unidirectional T650-35/PMR-15

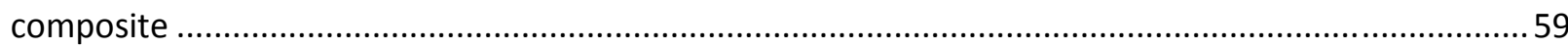

Figure 4-7: Shear stress vs. strain at different temperatures for unidirectional T650-35/PMR-15

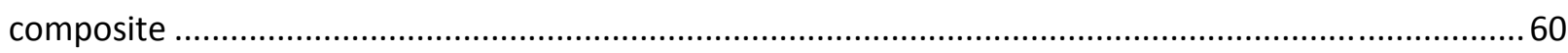

Figure 4-8: Transverse creep strain of unidirectional T650-35/PMR-15 composite at different temperatures ( $\sigma=1 \mathrm{MPa})$

Figure 4-9: a) Temperature ramp b) Transverse creep strain of unidirectional T650-35/PMR-15

composite under linear temperature ramp ( $\sigma=1 \mathrm{MPa})$.

Figure 4-10: a) Temperature cycle b) Transverse creep strain of unidirectional T650-35/PMR-15

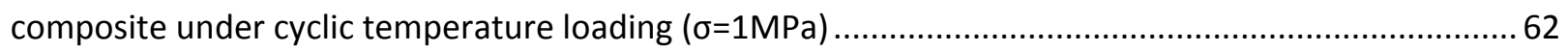

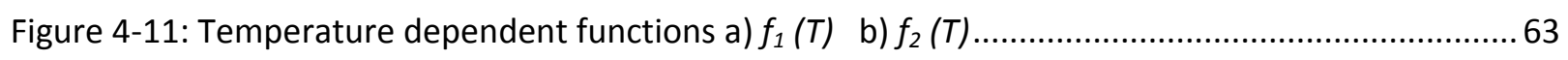

Figure 4-12: Isochronous stress-strain curves of T650-35/PMR-15 composite for different temperatures

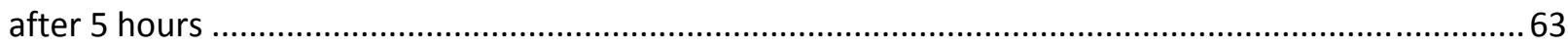

Figure 4-13: Difference between the elastic and viscoelastic response of T650-35/PMR-15 composite versus time at different temperatures. 64

Figure 4-14: Strain evolution upon loading-unloading and reloading for T650-35/PMR15 composite for different stress levels at $\mathrm{T}=250^{\circ} \mathrm{C}$.

Figure 4-15: Strain evolution upon loading-unloading and reloading for T650-35/PMR15 composite at

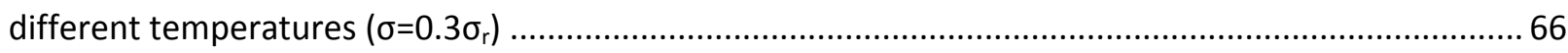

Figure 5-1: Shear creep compliance for $10^{\circ}$ off-axis $\mathrm{T} 300 / 934$ composite at $\mathrm{T}=160^{\circ} \mathrm{C} \ldots \ldots \ldots \ldots \ldots \ldots \ldots . . . . . . . . . . . . .71$

Figure 5-2: Creep Failure response of T300/934 composite in transverse and shear loading at $\mathrm{T}=160^{\circ} \mathrm{C}$

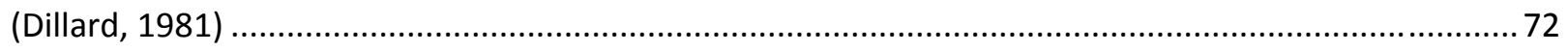

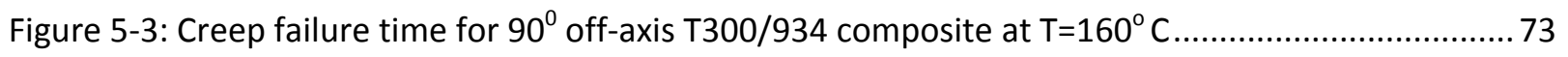

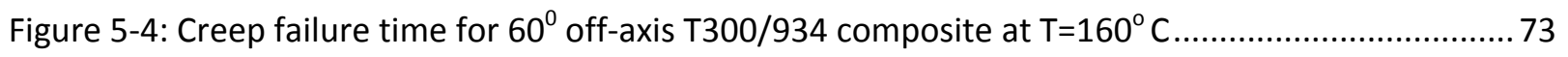

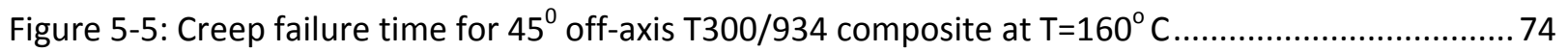

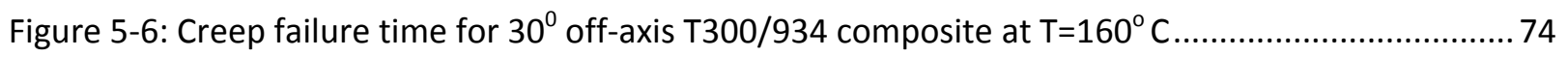

Figure 5-7: Creep failure time for $60^{\circ}$ off-axis T300/934 composite at two different temperatures ...... 75 
Figure 5-8: Creep failure time for $45^{\circ}$ off-axis T300/934 composite at two different temperatures ...... 76 Figure 6-1: Convergence algorithm to find fatigue life of the composite ............................................. 81

Figure 6-2: Axial creep strain for $60^{\circ}$ off-axis Glass/Epoxy composite ................................................. 83

Figure 6-3: Axial creep strain for $90^{\circ}$ off-axis Glass/Epoxy composite ..................................................83

Figure 6-4: Longitudinal fatigue failure diagram of E-Glass/Epoxy composite (Hashin and Rotem, 1973)

Figure 6-5: Transverse Fatigue and shear fatigue failure diagram of E-Glass/Epoxy composite (Hashin

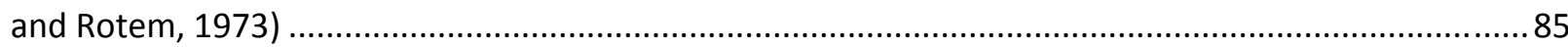

Figure 6-6: Fatigue failure diagram of off-axis Glass/Epoxy composite, $\theta=5^{\circ}$ and $\theta=10^{\circ} \ldots \ldots \ldots \ldots \ldots \ldots . . . . . . . . . . .86$

Figure 6-7: Fatigue failure diagram of off-axis Glass/Epoxy composite, $\theta=15^{\circ}$ and $\theta=20^{\circ} \ldots \ldots \ldots \ldots \ldots \ldots . . . . . . . . . .86$

Figure 6-8: Fatigue failure diagram of off-axis Glass/Epoxy composite, $\theta=30^{\circ}$ and $\theta=60^{\circ} \ldots \ldots \ldots \ldots \ldots \ldots . . \ldots 7$

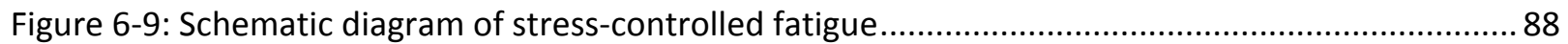

Figure 6-10: Maximum accumulated viscoelastic strain for the $60^{\circ}$ composite off-axis laminate............ 88

Figure 6-11: Fatigue failure diagram of off-axis Glass/Epoxy composite, $\theta=60^{\circ}$ with and without considering the effect of viscoelasticity

Figure 6-12: Transverse creep strain for T300/5208 unidirectional composite at different stress levels 91 Figure 6-13: Shear creep strain for $10^{\circ}$ off-axisT300/5208 unidirectional composite at different stress

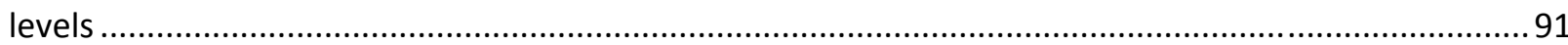

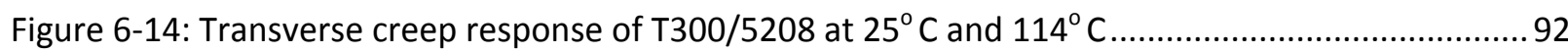

Figure 6-15: S-N diagrams of the composite material in principal directions at $25^{\circ} \mathrm{C}$ and $114^{\circ} \mathrm{C} \ldots \ldots \ldots . . . .93$

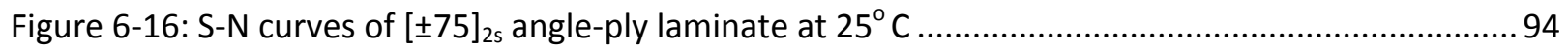

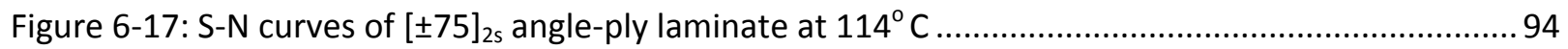

Figure 6-18: Transverse mean strain development due to fatigue and creep ....................................... 95

Figure 6-19: Contribution made by viscoelasticity to the total strain evolution at two different

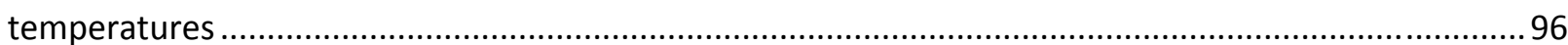

Figure 6-20: The effect of mean stress on the contribution of viscoelasticity to the total strain

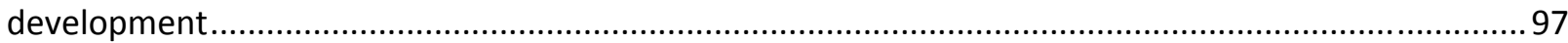

Figure 7-1: A sketch of the neat resin sample (All dimensions are in $\mathrm{mm}$ ) ........................................ 100

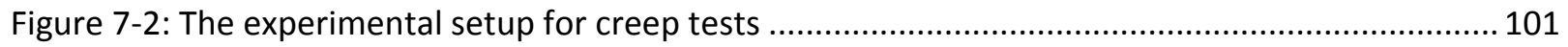

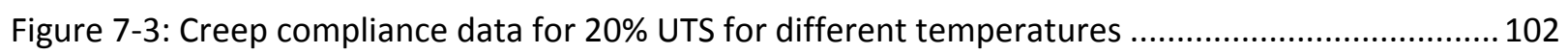

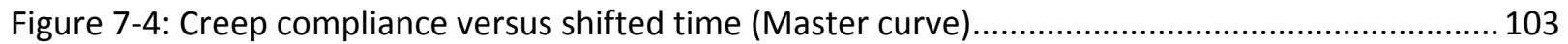

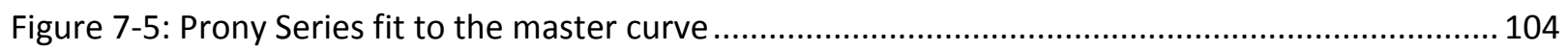

Figure 7-6: Creep response of the composite at $180^{\circ} \mathrm{C}$ for $30 \%$ of the UTS ...................................... 106

Figure 7-7: Creep response of the composite at $180^{\circ} \mathrm{C}$ for $60 \%$ of the UTS with the experimental data

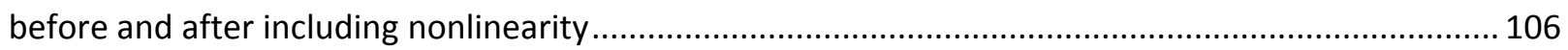

Figure 7-8: Creep response of the composite at $270^{\circ} \mathrm{C}$ for $30 \%$ of the UTS .......................................... 107

Figure 7-9: Creep response of the composite at $270{ }^{\circ} \mathrm{C}$ for $60 \%$ of the UTS with the experimental data

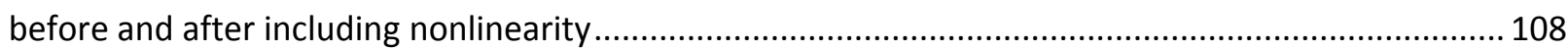

Figure 7-10: The mean strain evolution during the fatigue loading of the composite at $180^{\circ} \mathrm{C}$ for $40 \%$

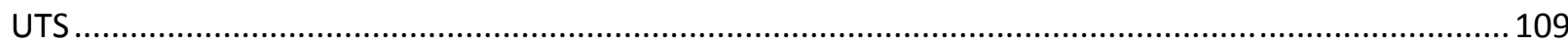

Figure 7-11: The mean strain evolution during the fatigue loading of the composite at $180{ }^{\circ} \mathrm{C}$ for $60 \%$ UTS. 109 
Figure 7-12: The mean strain evolution during the fatigue loading of the composite at $270{ }^{\circ} \mathrm{C}$ for $40 \%$

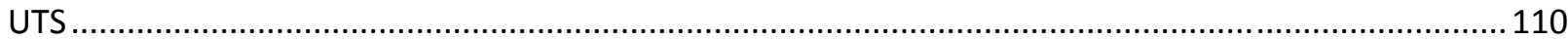




\section{CHAPTER 1: INTRODUCTION}

\subsection{Motivation}

High strength-to-weight ratios of composites compared to metallic materials has won them numerous applications in different industries. In particular, polymer matrix composites (PMC's) are being widely used in the aerospace industry. As an example, modern commercial aircrafts such as the Airbus 380 and Boeing 787 have utilized composite materials for their structural load bearing components. For instance, the Boeing 787 has a gross weight comprised of $50 \%$ composite resulting in an aircraft which is $80 \%$ volume in composite. Figure

1.1 shows an overview of the usage of composite materials in Boeing 787 commercial aircraft. One apparent advantage of using composite materials in an aircraft structure is decreasing the weight which will result in the reduction of fuel consumption.

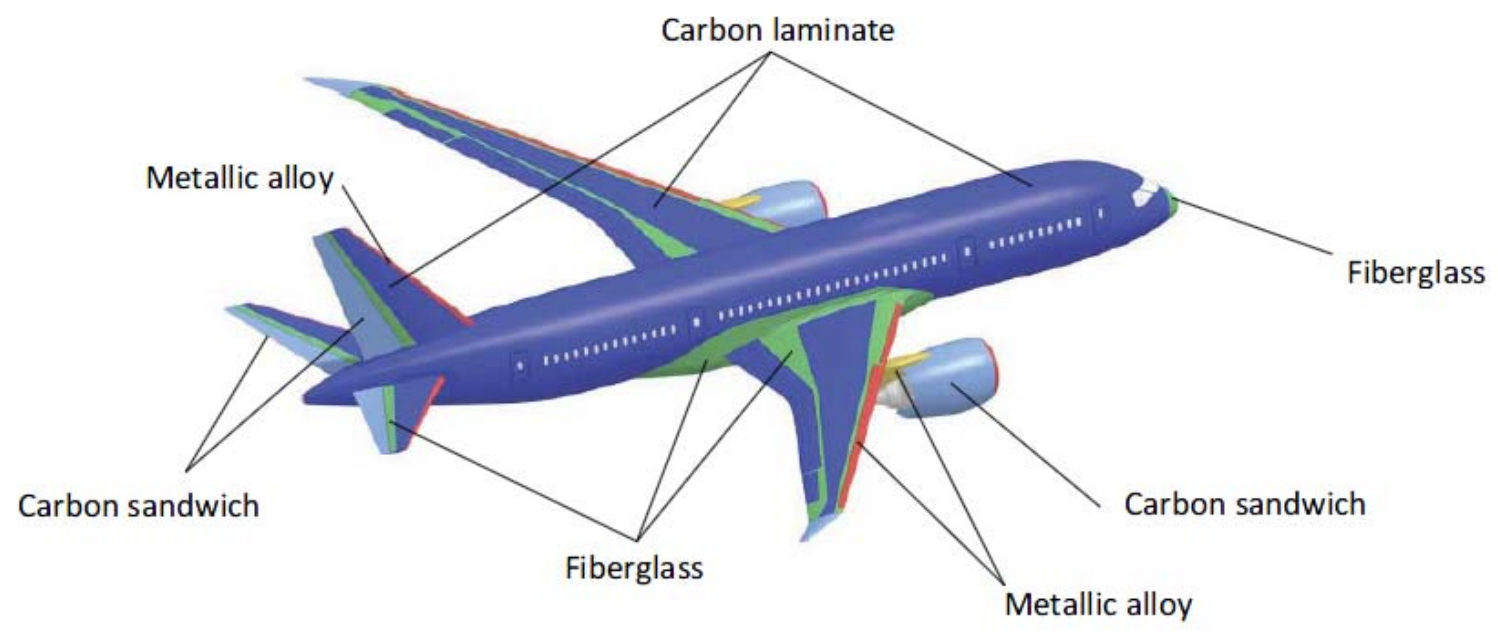

Figure 1-1: Boeing 787 material composition (Hawk, 2005) 
One important feature expected of polymer matrix composites in today's applications especially in advanced aerospace systems, is to sustain high temperatures and mechanical loadings while maintaining their light weight and flexibility. These applications usually involve long term exposure of the composite material to high temperatures such as in the propulsion systems and other engine components of commercial and military aircrafts. Examples of these applications include structural and non-structural parts on or around the aero-engines and airframe components of supersonic and hypersonic aircrafts and space vehicles, insulation for flexible cables to use as a high temperature adhesive in the semi-conductor industry and high temperature composite membranes for polymer electrolyte fuel cells. A great deal of attention has recently been focused on the use of composite materials in areas where the temperatures could be in the range of 100 to $400^{\circ} \mathrm{C}$.

One suitable candidate to be used for such applications are fiber reinforced polymer matrix composites (PMC's). It (Montesano et al., 2011) is mainly due to their unique combination of high-temperature stability, good chemical and solvent resistance as well as excellent retention of mechanical properties at elevated temperatures. However, the integration of PMC's into such applications is still not so widespread. As a result, high temperature polymer matrix composites have recently been the subject of numerous research studies.

Unlike metals in which damage starts in the form of micro cracks at loci of high stress concentration, there are different damage mechanisms in composite materials which may initiate throughout the structure without any explicit stress concentrations(Montesano et al., 2011). The existence of two or more distinct constituent phases in composite materials causes 
them to experience different forms of damage mechanisms which include matrix cracking, fiber fracture, fiber-matrix interface debonding and delamination between adjacent plies (Jen and Lee, 1998b). Due to the potentially large number of plies of different fiber orientations and perhaps material properties, determining a failure criterion of any degree of generality through experiments only, may seem to be an unrealistic task. On the other hand, in polymer matrix composites, the resin matrix material has moduli and strength properties which are viscoelastic or time dependent. This time dependent effect is further augmented by high temperature which accelerates damage progression and material property degradation. The viscoelastic behavior of the polymeric matrices of composite materials can significantly influence their deformation, strength and failure response under different loading conditions. Due to the hereditary effect of viscoelastic materials, the response to an applied load depends not only on the present state of loading but also on the previous states. In fact, one of the most important concerns arising in the use of polymers as the matrix component of fiber reinforced composites is the hereditary (viscoelastic) nature of the molecular structure of the particular polymer being used.

Applications of polymer matrix composites (PMC) in automotive and aerospace industries, bridge structures, water and waste systems and offshore explorations and oil productions, require a service life of 10 to 50 years (Guedes, 2007). On the other hand, performing experiments on the material or structure for such long periods is not economically feasible. An additional issue in the case of high temperature PMC's is that long term creep and fatigue testing at elevated temperatures usually limits the use of conventional testing equipment and techniques. In fact, there is an ever increasing demand for the development of suitable 
theoretical models for accelerated life characterization of PMC materials. Therefore development of a predictive model to study the response of high temperature PMC's to both static and dynamic loading, in particular the contribution of the time dependent effect of the matrix material to the failure of the composite system, poses a serious challenge.

\section{$1.2 \quad$ Research Objectives}

The main objective of the present thesis is to investigate the effect of temperature on the creep and fatigue behavior of polymer matrix composites through a simplified micromechanical approach. In particular, the role of viscoelasticity on the fatigue behavior of polymer matrix composites at room and high temperatures needs to investigated. In fact, regarding the limitations of conducting experiments at high temperatures, the present work aims to present a theoretical substitute which is validated through comparison with the existing experimental data in the literature as well as the experiments conducted by the author. To this end, the following tasks have been carried out:

- $\quad$ Programming a Simplified Unit Cell Micromechanical model in MATLAB.

- $\quad$ Studying the effect of temperature on the elastic and viscoelastic responses of PMR15 based composite systems at high temperatures based on the assumption of a thermorheologically complex material.

- Introducing a micromechanical approach to predict the creep failure of polymer matrix composite materials at high temperatures.

- Proposing a methodology to Investigate the role of viscoelasticity on the fatigue failure of polymer composite materials at room and high temperatures. 
- Carrying out creep and fatigue experiments to characterize the viscoelastic response of the RP46 high temperature resin based composite materials.

\subsection{Thesis Outline}

Chapter 2 presents a literature review of the creep and fatigue behavior of fiber reinforced polymer matrix composites including the effect of high temperature. Chapter 3 introduces the Simplified Unit Cell Micromechanical model and its formulation. In chapter 4 the thermomechanical viscoelastic response of a high temperature polymer matrix composite system made up of T650-35 graphite fibers embedded in PMR-15 resin is studied through the Simplified Unit Cell Micromechanical (SUCM) model. Chapter 5 introduces a micromechanical approach to study the time dependent failure of unidirectional polymer matrix composites. The composite material investigated is T300/934 which is suitable for service at high temperatures in aerospace applications. In chapter 6, the micromechanical model is modified to study the role of viscoelasticity on the fatigue behavior of polymer matrix composites EGlass/Epoxy and Graphite/Epoxy at room and high temperatures. Chapter 7 contains a casestudy on the viscoelasticity of the advanced high temperature RP46 polyimide resin based composite system. In chapter 8 , the general conclusions, contributions and suggestions for future work are presented. 


\section{CHAPTER 2: LITERATURE REVIEW}

\subsection{High Temperature Polymer Matrix Composites}

High temperature polymer matrix composites have recently been the focus of numerous research studies. In the 1970's and 1980's, NASA developed two types of polymers as candidates for use in high temperature composite materials. These two types are known as linear polyimides and addition aromatic polyimides (St. Clair and St. Clair, 1981). The main advantages of linear polyimides are their high damage tolerability and thermal stability over a wide range of temperatures. On the other hand, the addition aromatic polyimides are more brittle and possess higher temperature stability compared to the first group due to their crosslinked molecular structure (Meador, 1987). However, because of containing health hazardous by-products, these polymers were never largely produced and used for high temperature polymer matrix composites.

The first group of polymers widely produced by NASA for use in high temperature PMC's were developed using the polymerization of monomer reactants (PMR) approach (Serafini and Vannucci, 1975). These polyimides were found to have excellent thermal stability, ease of manufacturability and ability to withstand temperatures more than $300^{\circ} \mathrm{C}$. The main derivative of this kind of polyimide resins is PMR-15 with extensive applications in the aerospace industry. The maximum useful long term operating temperature of PMR-15 is about $260^{\circ} \mathrm{C}$ for jet engine applications. Two other high temperature polyimide resins developed by NASA are AMB-21 (Tiano et al., 2000) and DMBZ-15 (Xie et al., 2001). These thermoset polyimides have the same properties as PMR-15 but without any hazardous by-products. In 
fact, DMBZ-15 has a higher wear resistance compared to PMR-15 and is suitable for long term exposures to temperatures more than $300^{\circ} \mathrm{C}$.

Another example of high temperature polyimide resins is Avimid K3B which is a thermoplastic material used for supersonic transport aircrafts with an operating temperature of approximately $180^{\circ} \mathrm{C}$ (Sacks and Johnson, 1998). Other thermoset and thermoplastic polyimide resins considered for PMC matrix material for supersonic aircraft applications include R1-16, PETI-5 and PIXA (Shimokawa et al., 2008). In an effort to develop polyimides with higher service temperatures, NASA created RP-46 as a superior replacement for the existing polyimide resin systems. Its ability to resist extreme operating temperatures (up to $375^{\circ} \mathrm{C}$ ) makes it an ideal material for many critical aerospace applications that have little tolerance for failure.

Most recently, a thermoset polyimide called HFPE-II-52 was developed at NASA Glenn Research Center for use in high temperature composite materials (Bhargava, 2007).The maximum operating temperature for this polyimide is reported to be $315^{\circ} \mathrm{C}$. Although polyimide based polymer matrix composites (PiMC's) have many other improved properties compared to other PMC's, they are mainly used for applications where high specific strength, high specific stiffness and high temperature resistance are required. Examples of such applications are space launch propulsion systems, rocket engine components and advanced turbine engine applications (Thesken et al., 2004). More specifically, for instance, PMR-15 based composites are used in engine ducts for GE F404 engines, firewall in GE F90 engine and various splitters and fairings in F110 engines. 
Thanks to their light weight and corrosion resistance, PiMC's have also attracted a lot of applications in maritime structures such as displacement vessels, ships with single skin structures as well as in offshore including fire protection panels, water piping systems, partition walls, tanks and vessels (Meador, 1995). One important potential application of PMC's in general and high temperature PiMC's in particular, is the next generation jet engines currently under research in Pratt and Whitney and General Electric. These engines require greater durability and less weight for better operation while maintaining structural integrity (Bhargava, 2007). Polyimide's ability to from organometallic compounds with metals enables PiMC's to be used in high temperature applications which require them to be adhered to metal surfaces. In fact, PiMC's coefficient of thermal expansion can be adjusted so as to fit that of metal to minimize residual stresses during processing.

\subsection{Literature Review on the Creep Behavior of Fiber Reinforced Polymer Composites}

\subsubsection{Time dependent behavior of polymer matrix composite materials}

In polymer matrix composites, the resin matrix material has moduli and strength properties which are viscoelastic or time dependent. This time dependent effect is especially important at elevated temperatures which accelerate damage progression and material property degradation. Due to the hereditary effect of viscoelastic materials, the response to an applied load depends not only on the present state of loading but also on the previous states. In fact, one of the most important concerns arising in the use of polymers as the matrix component of fiber reinforced composites is the hereditary (viscoelastic) nature of the molecular structure of the particular polymer being used. The objective of this section is to review the literature on 
the creep behavior and creep failure of PMC's. The creep behavior of PMC's has been extensively investigated both experimentally and analytically. However, due to the immense number of works on the subject and since the focus of the present thesis is on a micromechanical approach, we only review some of the most popular and frequently cited theoretical models.

\subsubsection{Findley's power law}

Findley's power law model (Findley et al., 1976) is known to be of the most frequently used analytical models investigating the viscoelastic behavior of different composite materials. The general form of the power law equation is:

$\varepsilon(t)=\varepsilon_{0}^{\prime}+\varepsilon_{t}^{\prime} t^{n}$

Where

$\varepsilon(t):$ total time dependent strain

$\varepsilon_{0}^{\prime}$ : stress-dependent and temperature-dependent initial elastic strain

$\varepsilon_{t}^{\prime}:$ stress-dependent and temperature-dependent coefficient

$n$ : stress-independent coefficient

$t$ : time after loading

There is also another form of Findley's equation which is expressed in terms of creep compliance as follows:

$D(t)=\frac{\varepsilon(t)}{\sigma}=D_{0}+D_{t} t^{n}$

Where 
$D(t)=$ total time dependent creep compliance

$\sigma=$ the applied stress

$D_{0}=$ instantaneous creep compliance

$D_{t}=$ transient creep compliance

The effect of temperature of the viscoeastic response has been included in Findley's power law model by Yen and Morris (1989) in the following form:

$\varepsilon(\sigma, T, t)=\sigma\left[D_{0}(\sigma, T)+D_{t}(\sigma, T) t^{n}\right]$

\subsubsection{Boltzmann's superposition principle}

According to Boltzmann's principle the sum of the time-dependent strain outputs resulting from each component of stress input is the same as the strain output resulting from the combined stress inputs (Scott et al., 1995). This principle is expressed as:

$\varepsilon(t)=J_{0} \sigma+\int_{0}^{t} J(t-\tau) \frac{d \sigma(\tau)}{d \tau} d \tau$

Where

$J_{0}$ : time-independent component of creep compliance

$J(t)$ : time-dependent component of the creep compliance

The above-mentioned components are independent of stress which therefore makes this principle inapplicable to nonlinear viscoelastic materials.

\subsubsection{Schapery single integral model}

Schapery's single integral model is based on the principles of irreversible thermodynamics. The Schapery integral for the uniaxial state of stress is expressed by Schapery (1969): 
$\varepsilon(t)=g_{0} D_{0} \sigma+g_{1} \int_{0}^{t} D_{t}\left(\psi-\psi^{\prime}\right) \frac{d\left(g_{2} \sigma\right)}{d \tau} d \tau$

$\psi$ is the reduced time which is given by:

$\psi=\int_{0}^{t} \frac{d t^{\prime}}{a_{\sigma}}$

$\psi^{\prime}=\int_{0}^{\tau} \frac{\delta \tau^{\prime}}{a_{\sigma}}$

$g_{0}, g_{1}, g_{2}$ and $a_{\sigma}$ are called the nonlinear material parameters which are mainly stress and temperature dependent. For a linear viscoelastic material, these parameters are equal to unity. It should be noted that for the case of linear viscoelastic materials, the above-integral relation will be reduced to Boltzmann's superposition principle. Under a constant stress, the Schapery equation takes the following form (Lou and Schapery, 1971):

$\varepsilon(t)=g_{0} D_{0} \sigma+g_{1} D_{t}\left(\frac{t}{a_{\sigma}}\right) g_{2} \sigma$

Where $D_{t}$ is expressed in terms of Findley's power law:

$D_{t}=k t^{n}$

$D_{0}$ and $D_{t}$ can be determined from creep tests under low stresses i.e. when the material is in the linear viscoleastic domain. The nonlinear coefficients $g_{0}, g_{1}, g_{2}$ and $a_{\sigma}$ are calculated from creep and recovery data at different levels of stress. Another way to deal with Schapery's equation is through a recursive scheme which will be explained in detail in chapter 3 . The main advantage of this scheme is its ability to capture the hereditary effect in viscoelastic materials 
meaning that the response to an applied load depends not only on the present state of loading but also on the previous states

\subsubsection{Creep failure of polymer matrix composites}

The viscoelastic behavior of the polymeric matrices of composite materials can significantly influence their deformation, strength and failure response under different loading conditions. Due to the hereditary effect of viscoelastic materials, the response to an applied load depends not only on the present state of loading but also on the previous states. In fact, one of the most important concerns arising in the use of polymers as the matrix component of fiber reinforced composites is the hereditary (viscoelastic) nature of the molecular structure of the particular polymer being used. Applications of polymer matrix composites (PMC) in automotive and aerospace industries, bridge structures, water and waste systems and offshore explorations and oil productions, require a service life of 10 to 50 years (Guedes, 2007). On the other hand, performing experiments on the material or structure for such long periods is not economically feasible. An additional problem in the case of high temperature PMC's is that long term creep testing at elevated temperatures usually limits the use of conventional testing equipment and techniques. Therefore there is an ever increasing demand for the development of suitable theoretical models for accelerated life characterization of PMC materials. A great amount of research has been carried out on the development of time dependent failure criteria to predict the lifetime of polymers and polymer matrix composite materials. What follows is an introduction to some of the existing theoretical approaches to predict the life time of polymer matrix composites. 


\subsubsection{Kinetic rate theory approach to creep failure}

This approach is based on the kinetics of molecular flow and rupture of the atomic structure. Using this approach and upon conducting experiments on more than 50 different materials including both metals and polymers, Zhurkov (1984) proposed the following equation for the time to creep rupture in a uniaxial tensile test:

$$
D_{t}=t_{0} \exp \left(\frac{\left(U_{0}-\gamma \sigma\right)}{k T}\right)
$$

The parameter $t_{0}$ is defined as the natural oscillation period of the atomic structure which is a constant for all the materials. $k$ and $T$ denote Boltzman constant and absolute temperature, respectively. $U_{0}$ is the activation energy which should be overcome in order to rupture the atomic structure of the material. $v$ is a constant which depends on the previous treatments of the material and varies over a wide range for different materials.

\subsubsection{Fracture mechanics approach}

Several authors have done a lot of work extending the principles of fracture mechanics to viscoelastic material in order to predict the time dependent growth of cracks. Knauss (1970) proposed a nonlinear first order differential equation to describe the growth of a crack in a large viscoelastic plate subject to a constant stress far from the position of the crack. The differential equation contained the creep compliance and the surface energy of the material. Schapery $(1975 a, 1975 b, 1975 c)$ developed a theory to predict the time of fracture initiation and crack tip velocity in linear viscoelastic materials which he applied to an elastomer under simple and biaxial stress states. Christensen(1981) developed a formula for estimating the lifetime of polymers and polymer matrix composites under steady load. The necessary input 
parameters for this model are the viscoelastic creep function of the material, energy of the crack-generated surface layer and a characteristic failure dimension of the body. The method was applied to aramid-composite material strands and was found to be agreeable with experimental results. In a more recent study, Christensen (2002) used a kinetic crack growth theory developed for polymers to evaluate linear cumulative damage. The life time was defined as the time needed for an initial crack to grow large enough to cause instantaneous further propagation. The formulation applies only to central crack problems with quasi-static crack growth in polymeric materials in glassy elastic states.

\subsubsection{The time dependent yielding model}

According to this model developed by Nagdi and Murch (1963), the total strain is the sum of plastic and viscoelasic strains and a yield function $f$ is defined as follows:

$f\left(\sigma_{i j}, \varepsilon^{p}{ }_{i j}, \chi_{i j}, k_{i j}\right)=0$

Where $\sigma_{i j}$ is the stress tensor, $\varepsilon_{i j}^{p}$ is the plastic strain tensor, and $k_{i j}$ is a tensor representing strain hardening. In order to account for viscoelasticity in the yield function, a time dependent factor $\chi_{i j}$ was introduced. Later, Crochet (1966) gave a specific form to this time dependent factor:

$\chi=\left[\left(\varepsilon^{V}{ }_{i j}-\varepsilon^{E}{ }_{i j}\right)\left(\varepsilon^{V}{ }_{i j}-\varepsilon^{E}{ }_{i j}\right)\right]^{0.5}$

and defined a time dependent uniaxial yield function as

$\sigma_{y}=A+B\{\exp (-C \chi)\}$

with $A, B$ and $C$ as material constants. This approach was used by Crochet to perform a stress analysis on a viscoelastic/plastic internally pressurized hollow cylinder in a state of plain strain. 


\subsubsection{Energy based creep failure criteria}

This approach is based on the concept of the energy stored in the viscoelastic subject to loading. However, the concept of distortional energy as a failure criterion for an elastic material cannot be readily extended to viscoelastic material because in addition to energy storage, viscoelastic deformation involves energy dissipation which should as well, be taken into account. The Reiner-Weissenberg (R-W) theory is probably one of the earliest approaches to predicting creep-failure of viscoelastic materials. According to this approach, when a viscoelastic material is loaded (e.g. in creep), it both stores and dissipates energy. Therefore, the total deformation energy at any time is the sum of the free (stored) energy and the dissipative energy. The R-W theory suggests that the deviatric free (stored) energy is responsible for failure (Reiner and Weissenberg, 1939). Hiel (1983) has utilized a generalized Voigt-Kelvin model and the concept of free energy from the R-W theory to describe a one dimensional delayed (time dependent) failure criterion for polymer matrix composites. According to Brinson (1999), Bruller has demonstrated that if the Poisson ratio is assumed to be constant, the deviatric portion of the stored energy comprises $93 \%$ of the total deformation energy in a uniaxial tension test. In addition, since many polymers are very sensitive to bulk properties, Brinson (1999) reasons that the total stored energy could be a better indicator of failure. According to Hiel (1983), the stored energy in the springs, the dissipated energy in the dampers and the total energy of the generalized Voigt-Kelvin model representing a viscoelastic material under creep load are given by

$$
W_{\text {springs }}=\sigma_{0}^{2}\left\{\frac{D_{0}}{2}+\sum_{i=1}^{N} \frac{D_{i}}{2}\left(1-\exp \left(-\frac{t}{\tau_{i}}\right)\right)^{2}\right\}
$$




$$
W_{\text {dampers }}=\sigma_{0}^{2}\left\{\sum_{i=1}^{N} \frac{D_{i}}{2}\left(1-\exp \left(-\frac{2 t}{\tau_{i}}\right)\right)\right\}
$$

$$
W_{\text {total }}=\sigma_{0}^{2}\left\{\frac{D_{0}}{2}+\sum_{i=1}^{N} D_{i}\left(1-\exp \left(-\frac{t}{\tau_{i}}\right)\right)\right\}
$$

Based on the R-W theory, there is a limit to the energy stored in the material which is a constant and therefore regarded as a material property. If in Equation 2.14, time approaches zero and the applied stress is replaced with the static rupture stress i.e. $\sigma_{0}=\sigma_{R}$, then the maximum allowed free energy is calculated as:

$$
W_{\max }=\sigma_{R}^{2} \frac{D_{0}}{2}
$$

So that the R-W failure criterion can therefore be expressed as follows [30]:

$$
W_{\text {springs }}<W_{\max }
$$

\subsection{Literature Review on the Fatigue of Fiber Reinforced Polymer Composites}

\subsubsection{Fatigue damage modeling of fiber reinforced composite materials}

Today, due to their light weight and high strength as compared to metallic alloys, composite materials are frequently used in different industries. However, understanding the nature of cyclic or fatigue loading for the prediction of lifetime of fiber reinforced composite materials is of paramount importance. While there is enormous literature on the fatigue failure of metallic materials, modeling fatigue damage in composite materials has not yet been as thoroughly 
investigated. The existence of two or more distinct constituent phases in composite materials causes them to experience different forms of damage mechanisms which include matrix cracking, fiber fracture, fiber-matrix interface debonding and delamination between adjacent plies. As a result, there are some major differences between the fatigue of metals and fiber reinforced composites. Fatigue micro cracks in metallic components initiate at high stress concentration locations which gradually coalesce to form a large crack which will eventually lead to the failure of the component. However, except towards the very end of the fatigue life, no significant reduction of stiffness can be observed. On the other hand, in fiber reinforced composites, damage with significant loss of stiffness starts at a very early stage (Degrieck and Van Paepegem, 2001). Some of the most serious difficulties to be overcome in the fatigue life prediction of composite materials have been reported as follows:

- Different growing damage mechanisms for different stress levels (Barnard et al., 1985).

- Loading sequence is important i.e. high-low and low-high load sequences can result in considerably different damage growths (Hwang and Han, 1986a).

- The so-called cycle-mix effect which means that for the same total number of cycles, composites that experience small cycle blocks have a shorter average fatigue lives compared to laminates that are subjected to large cycle blocks (Farrow, 1989).

- The impact of frequency on the fatigue life, in particular for matrix dominated loading conditions, which is due to the general sensitivity of the matrix to the loading rate (Ellyin and El-Kadi, 1990).

- The effect of viscoelasticity of the polymer matrix contributes to the evolution of damage in the composite material. 
A workable classification of fatigue life prediction models for fiber reinforced polymer composites is based on the fatigue criteria proposed by Sendeckyj (1990). According to this classification, fatigue models can generally fall into three categories: The fatigue life models, residual stiffness/strength phenomenological models, and the progressive damage models.

\subsubsection{Fatigue life models}

The fatigue life models are based on the information obtained from S-N curves or Goodman diagram and introduce a failure criterion to determine the fatigue life of the specimen. One of the first models falling into this category was proposed by Hashin and Rotem (1973):

$$
\begin{aligned}
& \sigma_{A}=\sigma_{A}^{u} \\
& \left(\frac{\sigma_{T}}{\sigma^{u}{ }_{T}}\right)^{2}+\left(\frac{\tau}{\tau_{u}}\right)^{2}=1
\end{aligned}
$$

Where $\sigma_{A}, \sigma_{T}$ and $\tau$ denote, axial, transverse and shear stresses, respectively. The superscript $u$ indicates the ultimate stress. According to this model, the material is assumed to have failed upon the failure of one the constituents. A modified version of the afore-mentioned fatigue model was developed by Reifsnider and Gao (1991) in which the failure criteria for matrix dominated and fiber-dominated failure are expressed in terms of the average stresses $\sigma_{i j}^{f}$ and $\sigma_{i j}^{m}$ in the matrix and fiber constituents, respectively. The failure functions for this model are:

$$
\begin{aligned}
& \sigma^{f}{ }_{11}=X^{f} \\
& \left(\frac{\sigma^{m}{ }_{22}}{X^{m}}\right)^{2}+\left(\frac{\sigma^{m}{ }_{12}}{S^{m}}\right)^{2}=1
\end{aligned}
$$


$X^{f}$ is the fatigue failure functions of the fiber. $X^{m}$ and $S^{m}$ denote the fatigue failure functions of the unreinforced matrix material, respectively. These failure functions are functions of Stress ratio, frequency, number of cycles and temperature.

In another model proposed by Ellyin and El-Kadi (1990), the fatigue failure criterion was expressed in terms the strain energy density through a power law type relation of the form:

$$
\Delta W_{t}=k N^{\alpha}{ }_{f}
$$

Where the fatigue life $N_{f}$ is related to the total energy input $\Delta W_{t} . k$ and $\alpha$ are functions of fiber orientation angle. The strain energy was calculated based on the assumption of elastic plane stress.

Fawaz and Ellyin (1994) developed a semi-log linear relationship between the applied cyclic stress $\mathrm{S}$ and the number of cycles to failure $\mathrm{N}$ :

$$
\begin{aligned}
& S=m \log (N)+b \\
& S_{r}=m_{r} \log (N)+b_{r}
\end{aligned}
$$

The subscript $r$ refers to a chosen reference line. The relation between the two sets of material parameters $(m, b)$ and $\left(m_{r}, b_{r}\right)$ is expressed through functions $f$ and $g$ as follows:

$$
\begin{aligned}
& m=f\left(a_{1}, a_{2}, \theta\right) \cdot g(R) \cdot m_{r} \\
& b=f\left(a_{1}, a_{2}, \theta\right) \cdot b_{r}
\end{aligned}
$$

$R$ denotes the stress ration and $\theta$ is the fiber orientation angle. $a_{1}$ and $a_{2}$ are defined as: 


$$
\begin{aligned}
& a_{1}=\frac{\sigma_{y}}{\sigma_{x}} \\
& a_{2}=\frac{\tau_{x y}}{\sigma_{x}}
\end{aligned}
$$

The major drawback of this model is its sensitivity to the choice of the reference (Degrieck and Van Paepegem, 2001).

Jen and Lee (1998a,1998b) proposed a generalized multiaxial Tsai-Hill fatigue failure criterion

$$
M_{11}^{2}\left(\frac{\sigma_{x x}}{\bar{\sigma}_{11}}\right)^{2}+M_{22}^{2}\left(\frac{\sigma_{x x}}{\bar{\sigma}_{22}}\right)^{2}-M_{11} M_{22}\left(\frac{\sigma_{x x}}{\bar{\sigma}_{11}}\right)^{2}+M_{12}^{2}\left(\frac{\sigma_{x x}}{\bar{\sigma}_{12}}\right)^{2}=1
$$

In this model, all in-plane stresses are expressed in terms $\sigma_{x x}$, through stress transformations between the global and local axes coordinate systems, the stress ratios $R_{x x}, R_{y y}$ and $R_{x y}$ as well as the ratios $\alpha$ and 6 between $\sigma_{x x}$ and $\sigma_{y y}$ and $\sigma_{x x}$ and $\sigma_{x y}$, respectively, the fatigue strengths $\bar{\sigma}_{i i}$ are functions of frequency, stress ratio which need to be determined experimentally. A semi-empirical fatigue life prediction model was developed by Bond (1999) for variable amplitude loading of glass fiber reinforced composites in which the S-N curve was described as:

$$
\sigma_{\max }=b \cdot \log (N)+c
$$

Where $b$ and $c$ are 4 th order polynomial functions of the stress ratio $R$. Another empirical fatigue model was proposed by Epaarachchi and Clausen (2000):

$$
\left(\frac{\sigma_{u l t}}{\sigma_{\max }}-1\right) \frac{1}{(1-R)^{\gamma}} f^{\beta}=\alpha\left(N^{\beta}-1\right)
$$


Where $f$ is the frequency, $\gamma$ is a constant fixed to 1.6. Parameters $\alpha$ and $\beta$ are determined experimentally. None of the models falling into the category of fatigue life models have directly taken the effect of viscoelasticity and temperatures into account which is of primary importance in the case of polymer matrix composites.

\subsubsection{Residual stiffness/strength models}

This class of models is based on the stiffness or strength degradation during the fatigue loading in which the growth of a damage variable $D$ is expressed in terms of macroscopically observable properties. One very popular expression for the damage variable $D$ is

$$
D=1-\frac{E}{E_{0}}
$$

Where $E_{0}$ is the modulus of the virgin (undamaged) material.

Hwang and Han (1986b) introduced the concept of fatigue modulus F which is defined as the slope of the applied stress and the resultant strain at a specific cycle. Based on this concept, they proposed three different damage models. However, the third model proved to be in better agreement with experimental data. The damage variable in this model is defined as:

$$
D=\frac{r}{1-r}\left[\frac{F_{0}}{F(n)}-1\right]
$$

Failure takes place when $D$ equals unity and $r$ is the ratio of the applied cyclic stress to the ultimate static stress.

Yang et al (1990) developed a residual stiffness model for fiber-dominated composites as follows: 
$\frac{d E(n)}{d n}=-E(0) Q v n^{v-1}$

$Q$ and $v$ are two parameters which correlated by a linear equation. According to experimental data, $v$ may be expressed in the form of a linear function of the applied stress. It was later observed that this model cannot be readily applied to matrix dominated composites. This led to a modified version of this model in which $E$ was replaced with the fatigue modulus $F$. Broendsted et al. $(1997 a, 1997 b)$ developed a stiffness reduction model for the fatigue life prediction of glass fiber reinforced composites based on the experimental observations of wind turbine materials under cyclic loadings. According to this model:

$$
\frac{d\left(\frac{E}{E_{1}}\right)}{d N}=-K \cdot\left(\frac{\sigma}{E_{0}}\right)^{n}
$$

Where $E$ is the cyclic modulus after $N$ cycles, $E_{1}$ is the initial cyclic modulus and $E_{0}$ is the static modulus. $\sigma$ denotes the maximum stress and $K$ is a constant. Whitworth has developed several phenomenological models based on stiffness degradation, the most comprehensive of which (Whitworth, 1997) expresses the growth of degradation as follows:

$$
\frac{d E^{*}(n)}{d n}=-\frac{a}{(n+1)\left[E^{*}(n)\right]^{n-1}}
$$

$E^{*}(N)=E(n) / E(N)$ is the ratio of the residual stiffness i.e. after $n$ cycles to the failure stiffness. $a$ and $m$ are parameters which depend on the applied stress, loading frequency, and 
temperature and other environmental factors. Kawai (1999) introduced a model for the offaxis fatigue unidirectional carbon fiber reinforced composites:

$$
\frac{d \omega}{d N}=\frac{K\left(\sigma_{\max }^{*}\right)^{n}}{(1-\omega)^{k}}
$$

$K, n$ and $k$ are material constants and $\sigma^{*}{ }_{\max }$ is a non-dimensional effective stress defined as:

$$
\sigma_{\max }^{*}=\operatorname{Max}\left\{\left(\frac{\sigma_{11}}{X}\right)^{2}-\frac{\sigma_{11} \sigma_{22}}{X^{2}}+\left(\frac{\sigma_{22}}{Y}\right)^{2}+\left(\frac{\tau_{12}}{S}\right)\right\}
$$

Where $X, Y$ and $S$ are the static tensile strength, transverse strength and shear strength, respectively. Ramakrishnan and Jayaraman (1993) developed a stiffness-based degradation model based on the failure of the constituents i.e. fiber, matrix and the interface. The damage function $D$ is expressed as:

$$
\frac{E}{E_{c}}=1-\left[\frac{E_{m} V_{m}}{E_{c}}\left((1-f) \frac{\ln (N+1)}{\ln \left(N_{f}\right)}+f \frac{N}{N_{f}}\right)\right]
$$

In the above-mentioned equation, subscripts $m, f$, and $c$ refer to matrix, fiber and composite constituents, respectively. Terms $V_{f}$ and $E_{f}$ correspond to fiber volume fraction and elastic modulus, respectively and terms $V_{m}$ and $E_{m}$ denote matrix volume fraction and elastic modulus, respectively. The factor $f$ represents the fiber matrix interface strength parameter which varies between 1 (perfect bond) and 0 (debond). $r$ denotes the ratio of the applied stress to the ultimate tensile stress. This model was later extended by Shirazi and Varvani (2010) to account for the fiber angle orientation. As for residual strength models, one of the earliest models in this category which was introduced by Halpin et al (1973) assumes that the 
residual strength $R(n)$ is a monotonically decreasing function of the number of cycles $n$ whose rate of change can be expressed as follows:

$$
\frac{d R(n)}{d n}=\frac{-A(\sigma)}{m[R(n)]^{m-1}}
$$

Where $A(\sigma)$ is a function of the maximum cyclic stress $\sigma$ and $m$ is a constant. Daniel and Charewicz (1986) proposed a model based on the normalized change in residual strength to study fatigue damage accumulation in cross-ply graphite/epoxy laminates. According to this model:

$$
\left[\frac{1-f r}{1-s}\right]=g\left(\frac{n}{N}\right)
$$

$f r=F r / F O$ is the normalized residual stress, $s=\sigma_{a} / F O$ is the normalized applied cyclic stress, $N$ is the number of cycles to failure at $\sigma_{a}$ and $g(n / N)$ is a function of the normalized number of cycles. Extensive experimental and theoretical work by Schaff and Davidson (1997) led to the development of a strength-based spectrum fatigue loading model:

$$
R(n)=R_{0}-\left(R_{0}-S_{p}\right)\left(\frac{n}{N}\right)^{v}
$$

$R$ is the residual strength; $S_{p}$ is the magnitude of the peak stress and $v$ is a parameter which takes different values depending upon the rate of degradation:

$$
\begin{array}{ll}
v=1 & \text { For when the strength degradation is linear } \\
v>>1 & \text { For when the strength degradation is initially constant strength but }
\end{array}
$$


decreases drastically close to failure

$$
v<1 \quad \text { For when there is a rapid initial loss of strength }
$$

Caprino and D'Amore (1998) proposed a residual strength model based on four-point bending fatigue experiments conducted on a random continuous fiber-reinforced thermoplastic composite. According their model, the residual strength undergoes a continuous decay following a power law:

$$
\frac{d \sigma_{n}}{d n}=-a_{0} \cdot \Delta \sigma \cdot n^{-b}
$$

Where $\sigma_{n}$ is the residual strength after $n$ cycles, $\Delta \sigma=\sigma_{\max }-\sigma_{\min }$ to account for the stress ratio $R$ and $a_{0}$ and $b$ are constants. Whithworth (2000) developed a residual strength degradation model, based on a previously proposed residual stiffness model according to which:

$$
S_{R}^{\gamma}=S_{U}^{\gamma}-\frac{n}{N}\left(S_{U}^{\gamma}-S^{\gamma}\right)
$$

$S_{R}$ and $S_{U}$ denote the residual strength and the ultimate strength and $\gamma$ is a parameter. The main deficiency of this class of fatigue models is the absence of any physically-based criteria for damage prediction and their failure to account for the fiber matrix properties and the effect of loading history (Montesano et al., 2011).

\subsubsection{Progressive damage models}

The main difference between this class of models with residual stiffness/strength models is the choice of a damage variable based on the actual damage mechanism of the composite 
such as matrix crack, interface failure, fiber fracture or delaminations. Bergmann and Prinz (1989) modeled delamination in graphite/epoxy specimens as follows:

$$
\frac{d A_{i}}{d N}=c . f\left(G_{i t}\right)^{n}
$$

Where $A_{i}$ is the delaminated area, $G_{i t}$ denotes the maximum amplitude of the energy release rate and c and are experimentally determined values. Feng et al. (1997) proposed a model for fatigue damage life prediction based on matrix cracking in carbon fiber reinforced specimens. According to this model mode I of crack growth can be described by a modified version of Paris law:

$$
\frac{d A}{d N}=D G^{n}{ }_{\max }
$$

In the aforementioned equation, $A$ is the damage area due to matrix cracking, $N$ is the number of fatigue cycles, $G_{\max }$ is the maximum energy release rate in a fatigue cycle, and $D$ and $n$ are material constants. Another model based on matrix cracking was proposed by Henaff-Gradin et al. (2000) for cross-ply laminates. The damage growth under fatigue in this model is expressed as:

$$
\frac{d S}{d N}=A\left(\frac{G_{I}}{G_{\max }}\right)^{n}
$$

Where $S$ is the crack surface, $G_{l}$ is the strain energy release rate for the current crack density, $G_{\max }$ is the strain energy release rate at the initiation of the first crack in the matrix and $A$ and $n$ are constants determined from the experimental measurements of the crack density. Some progressive damage models relate the damage mechanism to the residual mechanical 
properties mainly stiffness or strength. One of the first models of this type is the shear lag model that calculates stiffness reduction due to matrix cracking. Highsmith and Reifsnider (1982) examined the edge replicas of crack patterns of various composite materials and observed that shear deformations in any ply were restricted to a thin region near the interfaces of that ply with its neighboring plies so that the transverse cracks extended only up to this region but not into it. These observations were incorporated into analytical differential equations to predict stiffness loss. A model relating stiffness reduction to crack growth for (0/90)s glass fiber reinforced laminates was developed by Ogin et al (1985). According to this model, it is postulated that crack growth rate is a power function of the stored elastic energy between two adjacent cracks in a transverse ply. The stiffness degradation rate due to transverse matrix cracking is then obtained as:

$$
\frac{-1}{E_{0}} \frac{d E}{d N}=A\left[\frac{\sigma_{\max }^{2}}{E_{0}^{2}\left(1-E / E_{0}\right)}\right]^{n}
$$

In which $\sigma_{\max }$ is the maximum fatigue stress level and $A$ and $n$ are constants. Assuming delamination to be the predominant damage mechanism, Beaumont (1990) proposed the following damage variable:

$$
D=\frac{A}{A_{0}}=2.857\left(1-\frac{E}{E_{0}}\right)
$$

Where $A$ is the delaminated area and $A_{0}$ is the total area between plies. The damage growth for a quasi-isotropic carbon fiber laminate was then defined as follows: 


$$
\left(\frac{d D}{d N}\right)_{R=0.1}=(9.2 e 5)\left(\frac{\Delta \sigma}{\sigma_{T S}}\right)^{6.4}
$$

$R$ is the stress ratio, $\Delta \sigma=\sigma_{\max }-\sigma_{\min }$ and $\sigma_{T S}$ is the tensile strength. The failure criterion to determine the failure value $D_{f}$ in this model was chosen to be the strain failure. Carswell (1988) proposed a model for the fatigue life prediction of unidirectional laminates in which the damage variable $D$ is related to the length of the matrix cracks in the laminate through the following damage growth rate:

$$
\left(\frac{d D}{d N}\right)=p \sigma_{c} \frac{D^{2}}{N}
$$

Where $p$ is a parameter and $\sigma_{c}$ is the stress amplitude. Spearing and Beaumont (1992a,1992b) carried out an extensive research on the tensile fatigue behavior of notched carbon/epoxy cross-ply laminates. The dominant damage modes were observed to be splitting in the $0^{0}$ plies, delamination between the $0^{\circ}$ and $90^{\circ}$ plies and transverse cracking in the $90^{\circ}$ plies. The damage was thus characterized by the split length / and delamination angle $\alpha$. Based on the fatigue crack growth law for isotropic materials, the split growth rate is defined as:

$$
\left(\frac{d l}{d N}\right)=\lambda_{3}\left(\frac{\Delta G}{G_{c}}\right)^{m / 2}
$$

Where $\lambda_{3}$ and $m$ are constants, $\Delta G$ is the driving force; $G_{c}$ is the damage growth resistance. For an incremental growth $\delta /$ of the split length, the energy absorbed in forming new crack surfaces is calculated as: 


$$
\delta E=G_{s} t \delta l+G_{d}(l \tan \alpha) \delta l
$$

$G_{s}$ is the absorbed energy per unit area of the split, $G_{d}$ is the absorbed energy per unit area of the delamination, $t$ is the thickness of the $0^{0}$ ply and $\alpha$ is the delamination angle at the split tip. A fatigue model based on microcracks in cross ply laminates was proposed by Caron and Ehlacher (1997) based on the assumption that $90^{\circ}$ plies can be discretized in sections with a random distribution of strength. In addition, it was assumed that Paris law can be used to model crack propagation and that the residual strength degradation follows:

$$
\frac{d \operatorname{Re}}{d N}=-C \operatorname{Re}^{3-\eta} \Delta S^{\eta}
$$

Where $R e$ is the residual strength, $C$ and $\eta$ are constants and $\Delta S$ is the stress range. Upon the estimation of the residual life using the foregoing equation, the stresses in the sections are calculated and compared to the residual strength through am iterative process. Once a section breaks, stresses are distributed and the residual life of each section is estimated. Another fatigue life prediction model called the generalized residual material property degradation model was proposed by Shokrieh and Lessard (2000a,2000b). In this model, Hashin-type fatigue failure criteria were used for seven damage modes: Fiber tension, fiber compression, fiber-matrix shearing, matrix tension, matrix compression, normal tension and normal compression. For all these damage modes, the corresponding material property is set to zero upon the occurrence of failure. Progressive damage models provide a more accurate tool for predicting the fatigue behavior of composites (Degrieck and Van Paepegem, 2001); however, 
the implementation of these models in numerical software specifically on a cycle by cycle basis will be computationally costly.

\subsubsection{Effect of elevated temperature on the fatigue behavior of polymer matrix composites}

In the foregoing section, some of the models developed to investigate the fatigue life of composite materials in general and polymer matrix composites particular were reviewed. In this section, the effects of high temperature on the fatigue behavior of PMC's are presented through a review of experimental and predictive studies in the literature.

\subsubsection{Experimental studies}

Lo et al (1993) conducted isothermal mechanical fatigue tests on carbon fiber /CSPI at $450^{\circ} \mathrm{C}$ and room temperature; CSPI being a modified polyimide developed at Shan Institute of Science and Technology with a glass transition temperature of $511^{\circ} \mathrm{C}$. Not surprisingly, the fatigue life decreased drastically at the elevated temperature. Branco et al (1994) carried out isothermal fatigue tests on a glass fiber reinforced Phnolic BJP 2018L composite at different temperatures the highest of which being $200^{\circ} \mathrm{C}$. It was observed that at higher temperatures the material stiffness decreased consistently, however, at room temperature, there was little stiffness loss up until close to failure. Through another study Branco et al (1996) found out that the manufacturing method (hand lay-up or pultrusion) could considerably affect the fatigue life of a specimen at high temperatures. Uematsu et al (1995) studied the delamination of fiber reinforced PEEK thermoplastic laminates due to fatigue loading at $200^{\circ} \mathrm{C}$. They concluded that delamination growth rate increases drastically as a result of high temperature. Another study on fatigue delamination was conducted by Sjogren and Asp (2002) on prepreg 
fiber-reinforced epoxy laminates subject to flexural and mixed mode bending fatigue loading at $100^{\circ} \mathrm{C}$. The critical and threshold energy release rate was found to have decreased with increasing temperature leading to an increase in the delamination growth rate. Gyekenyesi et al (1995) carried out an experimental study on a woven fiber-reinforced AMB21 polyimide resin matrix composite. They observed that in case of static tensile tests, temperature had little effect on the modulus and ultimate stress and strain. However, the effect of temperature $\left(255^{\circ} \mathrm{C}\right.$ in their experiments) on the fatigue life of the composite was seen to be significant. Another effect of high temperature was the ratcheting of the stress-strain curve during tension-tension fatigue which was attributed to fiber fracture, matrix cracking and viscoelastic deformation. The effect of time and temperature on the flexural behavior of unidirectional CFRP laminates under fatigue loading was studied by Miyano et al (1994). The fatigue behavior was observed to be significantly dependent on both temperature and loading frequency as a result of the viscelastic behavior of the matrix material. Case et al (1997) studied the fatigue behavior of notched unidirectional-ply fiber-reinforced K3B resin laminate at a temperature of $177^{\circ} \mathrm{C}$. Elevated temperature was seen to speed up delamination and matrix cracking. Castelli et al (1998) conducted thermo-mechanical fatigue tests on chopped fiber-reinforced PMR15 matrix composites at a maximum temperature of $260^{\circ} \mathrm{C}$. They concluded that thermomechanical loading does not degrade material properties as much compared fatigue testing at a constant temperature. Kawai et al (2001) studied the effect of temperature and the off-axis angle on the fatigue behavior of unidirectional fiber-reinforced polymer composites. It was found out the effect of high temperature became more prominent for off-axis loading where the matrix share of the load increases. Gregory and Spearing (2005) conducted static and 
fatigue tests on unidirectional fiber-reinforced $977-3$ resin at different temperatures the maximum of which being $149^{\circ} \mathrm{C}$. The toughness of the composite was seen to increase at higher temperatures when subjected to tensile testing in flexure probably due to the fiber bridging phenomenon. It was also observed that elevated temperature sped up delamination and crack propagation due to the decrease in the resin yield strength. The effect of high temperature on the fatigue behavior of notched woven fiber-reinforced 1053 epoxy laminates was investigated by Shimokawa et al (2007). The tests were conducted at $110^{\circ} \mathrm{C}$. The effect of high temperature on the strength degradation was found out to be minimal in tension-tension fatigue, however, the strength degradation in compression-compression and tensioncompression was significant because of the dependence of the laminate compressive strength on the matrix behavior. The experimentally based models are specific to certain types of composites so that the results cannot be readily generalized to other types of composite materials.

\subsubsection{Predictive models}

Predictive fatigue models taking the effect of high temperature into account may fall into one of the three categories previously mentioned i.e. the fatigue life models, residual stiffness/strength phenomenological models and the progressive damage models. However, the emphasis in this section is on the implementation of the effect of temperature into the fatigue life of the composite. Shah et al (1995) took into account the effect of temperature along with other factors including fiber longitudinal strength, exposure time, mechanical and thermal cyclic loading on the material property degradation through a multi-factor interaction equation (MFIE): 


$$
\frac{M_{P}}{M_{P O}}=\left(\frac{T_{g w}-T}{T_{g w}-T_{o}}\right)^{l}\left(1-\frac{\sigma}{S_{f}}\right)^{m}\left(1-\frac{\sigma_{T}}{S_{f} T_{f}}\right)^{n}\left(1-\frac{\sigma_{M} N_{m}}{S_{f} N_{m f}}\right)^{p}\left(1-\frac{\sigma_{T} N_{T}}{S_{f} N_{T f}}\right)^{q}
$$

The exponent of each parenthesis controls the amount of degradation of that very term. The nomenclature of the above-mentioned equation is as follows:

$M_{P}$ : A general material property, $M_{P O}$ : The reference material property, $T:$ Temperature, $S$ : Strength, $\sigma$ : Stress, $N$ : Number of cycles and $t$ : time

Superscripts:

$g_{w}$ : Wet glass transition temperature, $M$ : Mechanical load, $f$ : final condition, $T:$ Thermal cyclic load.

Miyano et al. (1997), Miyano et al. (2000) and Miyano et al. (2005) have proposed a formulation for fatigue strength of PMC's at elevated temperatures where the effect of viscoelasticity. According to this model, the fatigue life of a PMC material should be described as a combination of static fatigue that is the strength degradation under the action of the static load i.e. creep and degradation of the strength due to the cyclic component of the load. Since the creep loading is considered as fatigue loading with a stress ratio $R=1$ at any given frequency, the fatigue strength $\sigma_{f}\left(t_{f}, R, f, T\right)$ for an arbitrary frequency $(f)$, stress ratio $R$, and temperature $(T)$ is:

$$
\sigma_{f}\left(t_{f}, f, R, T\right)=\sigma_{f, R=1}\left(t_{f}, f, T\right)+\sigma_{f, R=0}\left(t_{f}, f, T\right)(1-R)
$$

where $\sigma_{f, R=1}$ and $\sigma_{f, R=0}$ are the fatigue strength for $R=1$ and 0 , respectively. The major assumptions of this model are: strength independence of the loading rate, the linear cumulative damage law for monotonic loading and linear dependence of fatigue strength on 
stress ratio. Kawai et al (2001) studied the off-axis behavior of unidirectional fiber-reinforced polymer matrix composite from a phenomenological viewpoint. In their model, fatigue damage was represented by two damage variables $\omega_{f}$ and $\omega_{m}$ for fiber and matrix, respectively. The growth equations for these damage variables were:

$$
\begin{aligned}
& \frac{d \omega_{f}}{d N}=K_{f}\left(\sigma_{\max }^{*}\right)^{n_{f}}\left(\frac{1}{1-\omega_{f}}\right)^{k_{f}} \\
& \frac{d \omega_{m}}{d N}=K_{m}\left(\sigma_{\max }^{*}\right)^{n_{m}}\left(\frac{1}{1-\omega_{m}}\right)^{k_{m}}
\end{aligned}
$$

$K, n$ and $k$ are material constants. $\sigma^{*}{ }_{\max }$ is the maximum value of the non-dimensional effective stress. The following fatigue life equations were derived from the above-mentioned equations.

$$
\begin{aligned}
& N_{f}=\frac{1}{\left(\sigma_{\text {max }}^{*}\right)^{n_{f}}} \\
& N_{f}=\frac{1}{\left(\sigma_{\text {max }}^{*}\right)^{n_{m}}}
\end{aligned}
$$

Mahieux et al (2001) incorporated the effects of temperature on the strength and stiffness of unidirectional polymer matrix composites into a fatigue model developed by Reifsnider and Stinchomb ( 1986). The fatigue model is based on a residual strength integral expression defined as:

$$
F r=1-\int_{0}^{\tau}(1-F a(\tau)) j \tau^{j-1} d \tau
$$


The above equation relates the remaining strength $F r$ to $F a$, a failure function which is defined as the ratio between the maximum applied strain in bending fatigue and the ultimate strain. $\tau$ is a characteristic time which for fatigue loading is $\tau=n / N_{f}$ and $j$ is a material parameter. The effect of temperature is assumed to be on the polymer matrix modulus in accordance with:

$$
E_{m}(T)=E_{N} \exp \left(-\left(\frac{T}{T_{N}}\right)^{m_{N}}\right)+\sum_{i=1}^{N-1}\left(E_{i}-E_{i+1}\right) \exp \left(-\left(\frac{T}{T_{i}}\right)^{m_{i}}\right)
$$

$K$ is the number of property transition in the polymer matrix; $T_{i}$ and $m_{i}$ are the transition temperature and the statistical coefficient for the ith transition, respectively. Jen et al (2006) proposed a semi-empirical fatigue model for notched AS-4/PEEK APC-2 polymer matrix composite laminates at elevated temperatures up to $150^{\circ} \mathrm{C}$ as follows:

$$
\frac{B}{\sigma_{0}}=\left(\frac{d}{W}\right)^{-1}\left\{\left[\frac{T_{m}-T_{T}}{T_{m}-T_{0}}\right]^{0.5}-\left(\frac{\sigma}{\sigma_{0}}\right)\right\}
$$

$d / W$ is the ratio of the hole diameter $d$ to the specimen width. $T_{m}$ is the melting temperature, $T_{T}$ is the test temperature. $\sigma_{0}$ and $\sigma$ denote the ultimate strength at room temperature and maximum stress at the elevated temperature, respectively.

The above-given survey of the works in the area shows that the bulk of the research studies conducted on high temperature polymer matrix materials are experimental. Applications of polymer matrix composites in automotive and aerospace industries usually involve the exposure of the material to high temperatures for lengthy periods of time.

There are two major problems associated with experimental investigation of PMC's at elevated temperatures. First, performing experiments on the material or structure for such 
long periods is not economically feasible. Secondly, elevated temperature limits the use of conventional testing equipment and techniques. That's why there is an ever increasing demand for the development of suitable theoretical models for accelerated characterization of PMC materials subject to high temperatures. Analysis and prediction of the behavior of the composite material demand the knowledge of the behavior of each of the constituent components. On the other hand, at elevated temperatures the viscoelastic effects of the polymer matrix are more significant. The major drawback of the existing analytical models is their failure to directly account for the viscoelasticity of the matrix material in particular its role on the on the fatigue failure of polymer matrix composites. In addition, these models heavily depend on experimental parameters which thus makes them too specific usually applicable to one particular class of PMC's.

Regarding the aforementioned shortcomings, the present research work focuses on two main objectives: first, studying the effect of temperature on the elastic and viscoelastic response of polymer matrix composites, and second, proposing a methodology to investigate the role of viscoelasticity on the fatigue failure of polymer matrix composite materials at room and high temperatures through a micromechanical approach. 


\section{CHAPTER 3: SIMPLIFIED UNIT CELL MICROMECHANICAL MODEL}

Analytical Research studies conducted so far on the response of polymer matrix composites are based on macromechanics which predict the failure of composite as a bulk material. However, failure in polymer matrix materials is a localized phenomenon which demands a sufficiently accurate stress analysis of the representative volume element. That's why micromechanical analysis gives a far better insight into the failure mechanisms of the composite materials. In fact, through micromechanics, the modes of failure in fiber and matrix can be separately identified which helps modify the stiffnesses of the constituents accordingly. In particular, since at high temperatures, the general behavior of the polymer matrix composite is governed by matrix viscoelasticity, the need for micromechanical analysis becomes more crucial. Other advantages of micromechanics include the study of the effects of thermal residual stresses and reinforcement volume fraction.

No work has hitherto taken into account the viscoelastic behavior of the matrix material of PMC's within a micromechanical framework to study the response of polymer matrix composites at high temperatures. In reality, fibers are randomly distributed within the matrix material. However, for the sake of brevity, in the micromechanical model, a representative volume element (RVE) is defined such that it represents a small repeatable area of the cross section of the composite with the same effective properties as those of the composite system. One of the early analytical models based on the concept of RVE, is the Method of Cells (MOC) originally developed by Aboudi (1982) in which for the first time, the fiber cross section was assumed to be square. MOC, although well-known, is mathematically rigorous (Robertson and Mall, 1993,1994). 
The Simplified Unit Cell Micromechanical (SUCM) model is a simplified form of the Method of Cells (MOC) developed by Aghdam et al. (2000) to study the collapse behavior of metal-matrix composites under thermo-mechanical loading as well as modeling the interface damage of metal-matrix composites subject to transverse loading. While maintaining the same level of accuracy as MOC, the SUCM has a much simpler mathematical procedure. The main advantage of the present micromechanical model lies in its ability to give closed form solutions for the effective nonlinear response of unidirectional composites and to predict the material response to any combination of shear and normal loading. Although both SUCM and MOC yield similar results for the overall elastic moduli of unidirectional composites, the SUCM has, in fact, been found to give a better prediction for the overall transverse Poisson ratio (Aghdam and Dezhsetan, 2005). The model was later extended to include the nonlinear viscoelastic properties in closed form functions (Falahatgar et al., 2009).

\subsection{Matrix Material Constitutive Equation}

The matrix material is modeled based on Schapery's single integral constitutive equation in which the integral part can be calculated through a recursive scheme. The total strain can therefore be written as (Haj-Ali and Muliana, 2004b):

$$
\begin{aligned}
& \varepsilon(t)=\left[g_{0}{ }^{t} D_{0}+g_{1}{ }^{t} g_{2}{ }^{t} \sum_{n=1}^{N} D_{n}\left(1-\frac{1-\exp \left(-\lambda_{n} \Delta \psi^{t}\right)}{\lambda_{n} \Delta \psi^{t}}\right)\right] \sigma(t) \\
& -g_{1}{ }^{t} \sum_{n=1}^{N} D_{n}\left[\exp \left(-\lambda_{n} \Delta \psi^{t}\right) q_{n}{ }^{t-\Delta t}-\frac{1-\exp \left(-\lambda_{n} \Delta \psi^{t}\right)}{\lambda_{n} \Delta \psi^{t}} g_{2}{ }^{t-\Delta t} \sigma(t-\Delta t)\right]
\end{aligned}
$$

Where $D_{0}$ is the uniaxial elastic compliance, $N$ is the number of terms, $D_{n}$ is nth coefficient of the Prony series and $\lambda_{n}$ is the $n$th reciprocal of the retardation time. $g_{0}, g_{1}$ and $g_{2}$ are the 
nonlinear material properties which are in general stress dependent. Also the reduced time increment is defined by:

$$
\Delta \psi^{t}=\psi^{t}-\psi^{t-\Delta t}=\int_{t-\Delta t}^{t} \frac{d \xi}{a(\sigma, T)}
$$

and the hereditary integral $q_{n}{ }^{t}$ for every term of the Prony series at the end of the current time is calculated as follows:

$$
q_{n}^{t}=\left[\exp \left(-\lambda_{n} \Delta \psi^{t}\right) q_{n}{ }^{t-\Delta t}+\frac{1-\exp \left(-\lambda_{n} \Delta \psi^{t}\right)}{\lambda_{n} \Delta \psi^{t}}\left(g_{2}{ }^{t} \sigma(t)-g_{2}{ }^{t-\Delta t} \sigma(t-\Delta t)\right)\right]
$$

The parameter $a(\sigma, T)$ is the total time shift factor which is measured with respect to the reference stress and temperature. The total time shift factor can be assumed to be in the following form (Walrath, 1991):

$$
a(\sigma, T)=a_{\sigma}(\sigma) a_{T}(T)
$$

The nonlinear parameters in Schapery's model, $g_{0}(\sigma)=g_{1}(\sigma)=g_{2}(\sigma)=a(\sigma)=1$ when the applied stress is sufficiently small, i.e. the material exhibits linear behavior (Lai and Bakker, 1996). If the temperature effects can be accounted for only through the shift factor $a_{T}(T)$, the material is called thermorheologically simple (TSM), otherwise it is classified as a thermorheologically complex material (TCM).The multiaxial material constitutive equation for the matrix material is (Falahatgar et al., 2010):

$$
\begin{aligned}
& \varepsilon_{i j}(t)=\left(1+v_{m}\right) S_{c}(t) \sigma_{i j}(t)-v_{m} S_{c}(t) \sigma_{l l}(t) \delta_{i j}+\left(1+v_{m}\right) R_{i j}(t)-v_{m} R_{l l}(t) \delta_{i j} \\
& i, j=1,2,3
\end{aligned}
$$


The matrix instantaneous creep compliance $S_{c}(t)$ and the hereditary creep strains Rij(t) are defined as:

$$
\begin{aligned}
& S_{c}(t)=\left[g_{0}{ }^{t} D_{0}+g_{1}{ }^{t} g_{2}{ }^{t} \sum_{n=1}^{N} D_{n}\left(1-\frac{1-\exp \left(-\lambda_{n} \Delta \psi^{t}\right)}{\lambda_{n} \Delta \psi^{t}}\right)\right] \\
& R_{i j}(t)=-g_{1}{ }^{t} \sum_{n=1}^{N} D_{n}\left[\exp \left(-\lambda_{n} \Delta \psi^{t}\right) q_{n, i j}{ }^{t-\Delta t}-\frac{1-\exp \left(-\lambda_{n} \Delta \psi^{t}\right)}{\lambda_{n} \Delta \psi^{t}} g_{2}{ }^{t-\Delta t} \sigma_{i j}(t-\Delta t)\right]
\end{aligned}
$$

\subsection{Assumptions of the Micromechanical Model}

As mentioned earlier, in the SUCM model, it is assumed that the composite material can be represented by rectangular cross section fiber and matrix subcells. Figure 3.1 shows the corresponding RVE of the composite lamina. As can be seen, due to symmetry the quarter unit cell is divided to four subcells, one fiber and three matrix subcells. The fiber i.e. the longitudinal direction is assumed to be along the $x_{1}$ axis and the other two axes, $x_{2}$ and $x_{3}$, represent the transverse directions. One major assumption in the SUCM model is that the displacement components are linear which results in constant stress and strain within the subcells. It has been shown by Aboudi (1982) that this is a sufficiently accurate assumption for modeling composites subjected to different loading modes. It is also assumed that normal and shear loadings can be dealt with independently from one another. This indicates that the normal stress in the RVE does not induce any shear stresses in the subcells. 


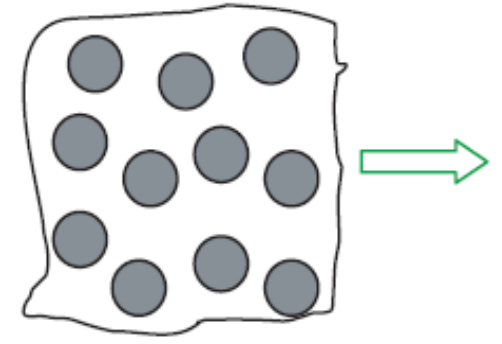

Real composite

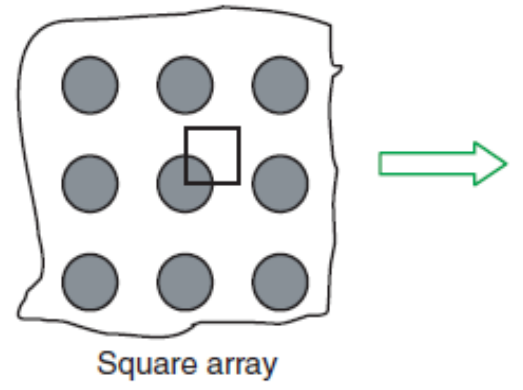

Square array

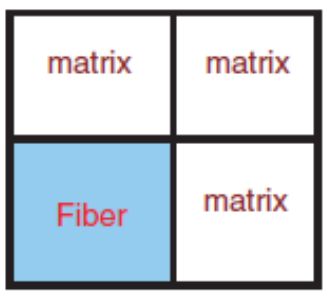

RVE

Figure 3-1: The Representative Volume of the Composite

\subsection{Normal Response of the Composite}

Figure 3.2 shows the RVE under normal transverse stresses. Assuming that the subcells are perfectly bonded the compatibility of displacements in the RVE can be written as follows:

$$
\begin{aligned}
& \varepsilon^{I}{ }_{11}=\varepsilon^{I I}{ }_{11}=\varepsilon^{I I I}{ }_{11}=\varepsilon^{I V}{ }_{11}=\bar{\varepsilon}_{11} \\
& a \varepsilon_{22}^{I}+b_{2} \varepsilon^{I I}{ }_{22}=a \varepsilon^{I I I}{ }_{22}+b_{2} \varepsilon^{I V}{ }_{22}=\bar{\varepsilon}_{22}\left(a+b_{2}\right) \\
& a \varepsilon^{I}{ }_{33}+b_{3} \varepsilon^{I I}{ }_{33}=a \varepsilon^{I I I}{ }_{33}+b_{3} \varepsilon^{I V}{ }_{33}=\bar{\varepsilon}_{33}\left(a+b_{3}\right)
\end{aligned}
$$

In the above-mentioned equations $\varepsilon^{k}{ }_{i i}$ denotes micro or local strains in the kth subcell and $\bar{\varepsilon}_{i i}$ represents the macro or global strains as a result of the applied macro or global stresses $\bar{\sigma}_{i i}$. Traction continuity conditions at the interfaces between the subcells are satisfied provided that:

$$
\sigma_{22}^{I}=\sigma_{22}^{I I}, \sigma_{22}^{I I I}=\sigma_{22}^{I V}, \sigma_{33}^{I I I}=\sigma_{33}^{I}, \sigma_{33}^{I I}=\sigma_{33}^{I V}
$$

Where $\sigma^{k}{ }_{i i}$ are the micro or local stresses in the kth subcell. The equilibrium equations of the RVE for normal loading in the three directions are as follows: 


$$
\begin{aligned}
& a^{2} \sigma_{11}^{I}+a b_{2} \sigma_{11}^{I I}+a b_{3} \sigma_{11}^{I I I}+b_{2} b_{3} \sigma_{11}^{I V}=\bar{\sigma}_{11}\left(a+b_{2}\right)\left(a+b_{3}\right) \\
& a \sigma_{22}^{I I}+b_{3} \sigma_{22}^{I V}=\bar{\sigma}_{22}\left(a+b_{3}\right) \\
& a \sigma_{33}^{I I I}+b_{2} \sigma_{33}^{I V}=\bar{\sigma}_{33}\left(a+b_{2}\right)
\end{aligned}
$$

The fiber material is assumed to be linearly elastic and transversely isotropic whereas the matrix is assumed to be viscoelastic. Equation 3.5 denotes the constitutive equation for matrix material which takes the following form under normal loading:

$$
\begin{aligned}
& \varepsilon^{k}{ }_{i j}(t)=\left(1+v_{m}\right) S_{c}^{k}(t) \sigma_{i j}^{k}(t)-v_{m} S_{c}^{k}(t) \sigma_{l l}^{k}(t)+\left(1+v_{m}\right) R_{i j}^{k}(t)-v_{m} R_{l l}^{k}(t) \\
& i=j=1,2,3
\end{aligned}
$$

Applying Equations 3.8-3.15 will result in a system of 8 equations and 8 unknowns as:

$$
A \sigma=F+H
$$

Where $A$ is the coefficient matrix $\sigma$ is the normal micro-stress vector, $H$ is the hereditary integral function vector and $F$ denotes the external load vector. The details of these matrices are given below:

$$
\begin{aligned}
& \sigma=\left\{\begin{array}{lllllllll}
\sigma_{11}{ }^{I} & \sigma_{22}{ }^{I I} & \sigma_{11}{ }^{I I} & \sigma_{33}{ }^{I I I} & \sigma_{11}{ }^{I I I} & \sigma_{22}{ }^{I V} & \sigma_{33}{ }^{I V} & \sigma_{11}{ }^{I V}
\end{array}\right\}^{T}
\end{aligned}
$$

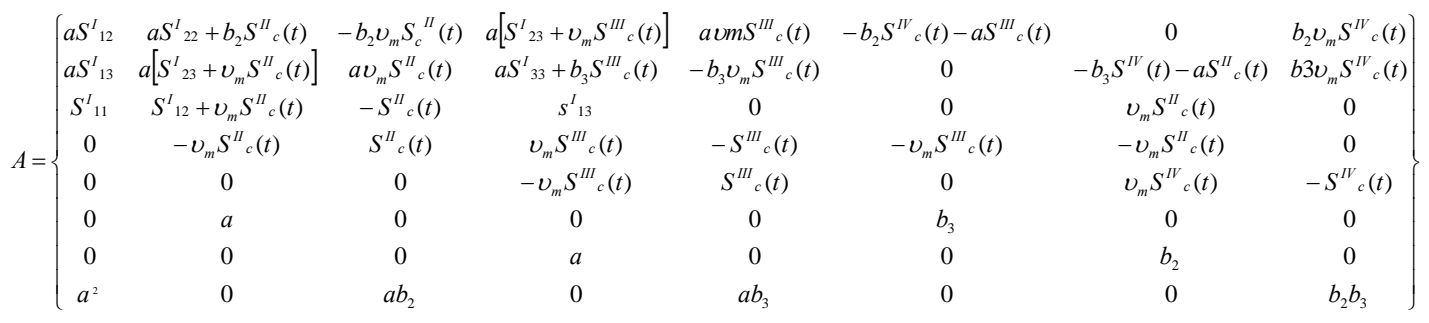

$$
\begin{aligned}
& F=\left\{\begin{array}{llllllll}
0 & 0 & 0 & 0 & 0 & \bar{\sigma}_{2}\left(a+b_{3}\right) & \bar{\sigma}_{3}\left(a+b_{2}\right) & \bar{\sigma}_{1}\left(a+b_{2}\right)\left(a+b_{3}\right)
\end{array}\right\}^{T}
\end{aligned}
$$




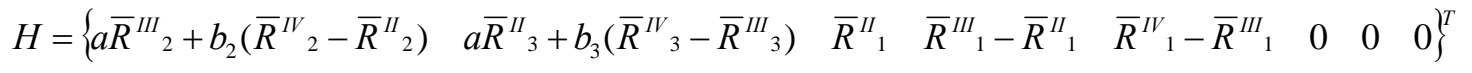

$$
\begin{aligned}
& \bar{R}_{i}=R_{i i}(t)-v\left[R_{j j}(t)-R_{k k}(t)\right] \quad i=1,2,3 \quad j=2,3,1 \quad k=3,1,2
\end{aligned}
$$

The global normal strains in the RVE can therefore be obtained as follows:

$$
\begin{aligned}
& \bar{\varepsilon}_{11}=S_{11}^{I} \sigma_{11}^{I}+S_{12}^{I} \sigma^{I I}{ }_{22}+S_{13}^{I} \sigma^{I I I}{ }_{33} \\
& \bar{\varepsilon}_{22}=\frac{1}{a+b_{2}}\left[\left(a S^{I}{ }_{22}+b_{2} S^{I I}{ }_{c}\right) \sigma^{I I}{ }_{22}+a\left(S^{I}{ }_{23} \sigma^{I}{ }_{33}+S_{12}^{I} \sigma^{I}{ }_{11}\right)-v_{m} b_{2} S^{I I}{ }_{c}\left(\sigma^{I I}{ }_{33}+\sigma^{I I}{ }_{11}\right)+\right. \\
& \left.b_{2}\left(R^{I I}{ }_{22}-v_{m}\left(R^{I I}{ }_{33}+R^{I I}{ }_{11}\right)\right)\right] \\
& \bar{\varepsilon}_{33}=\frac{1}{a+b_{3}}\left[\left(a S^{I}{ }_{33}+b_{3} S^{I I I}{ }_{c}\right) \sigma^{I I I}{ }_{33}+a\left(S_{13}^{I} \sigma_{11}^{I}+S^{I}{ }_{23} \sigma_{22}^{I}\right)-v_{m} b_{3} S^{I I I}{ }_{c}\left(\sigma^{I I I}{ }_{22}+\sigma^{I I I}{ }_{11}\right)+\right. \\
& \left.b_{3}\left(R^{I I I}{ }_{33}-v_{m}\left(R^{I I I}{ }_{22}+R^{I I I}{ }_{11}\right)\right)\right]
\end{aligned}
$$

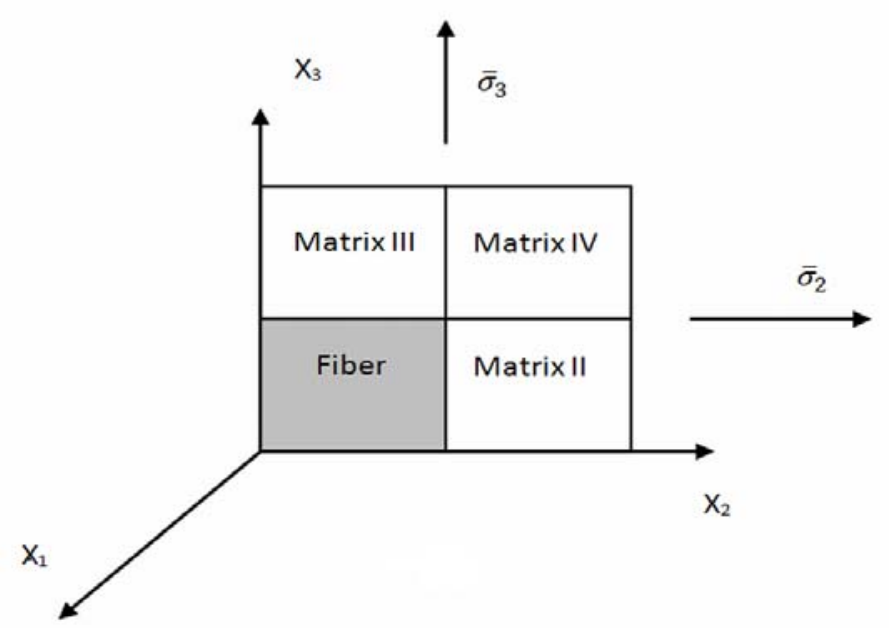

Figure 3-2: The RVE subject to normal loading 


\subsection{Shear Response of the Composite}

Figure 3.3 shows the RVE under shear loading. Similar to normal loading, the equilibrium and continuity equations need to be satisfied in the case of shear loading. The Equilibrium requirement relates the local and global shear stresses as follows:

$$
\begin{aligned}
& a \tau_{12}^{I}+b_{3} \tau_{12}^{I I I}=\left(a+b_{3}\right) \bar{\tau}_{12} \\
& a \tau_{13}^{I}+b_{2} \tau_{12}^{I I}=\left(a+b_{2}\right) \bar{\tau}_{13} \\
& \tau_{23}^{I}=\tau_{23}^{I I}=\tau_{23}^{I I I}=\tau_{23}^{I V}=\bar{\tau}_{23}
\end{aligned}
$$

Where $\bar{\tau}_{i j}$ represent the applied global shear stresses. Also, the equilibrium of local stress components at the subcell interfaces requires that:

$$
\tau_{12}^{I}=\tau_{12}^{I I}, \tau_{12}^{I I I}=\tau_{12}^{I V}, \tau_{13}^{I I I}=\tau_{13}^{I}, \tau_{13}^{I I}=\tau_{13}^{I V}
$$

The strain compatibility conditions are expressed as:

$$
\begin{aligned}
& a \gamma_{12}^{I}+b_{2} \gamma_{12}^{I I}=a \gamma_{12}^{I I I}+b_{2} \gamma_{12}^{I V}=\left(a+b_{2}\right) \bar{\gamma}_{12} \\
& a \gamma_{13}^{I}+b_{3} \gamma_{13}^{I I I}=a \gamma_{13}^{I I}+b_{3} \gamma_{13}^{I V}=\left(a+b_{3}\right) \bar{\gamma}_{13} \\
& a\left(a \gamma_{23}^{I}+b_{2} \gamma_{23}^{I I}\right)+b_{3}\left(a \gamma_{23}^{I I I}+b_{2} \gamma_{23}^{I V}\right)=\left(a+b_{3}\right)\left(a+b_{2}\right) \bar{\gamma}_{23}
\end{aligned}
$$

Knowing that $\gamma^{k}{ }_{i j}=2 \varepsilon^{k}{ }_{i j}$ when $i \neq j$, Equation 3.5 takes the following form for the case of shear loading:

$$
\begin{aligned}
& \gamma_{i j}^{k}(t)=2\left(1+v_{m}\right) S_{c}^{k}(t) \tau_{i j}^{k}(t)+2\left(1+v_{m}\right) R_{i j}^{k}(t) \\
& i \neq j
\end{aligned}
$$


Once the constitutive relation (Equation 3.32) is substituted into the strain compatibility equations, one can calculate $\tau_{12}^{I}$ and $\tau_{12}^{I I I}$ from the simultaneous solution of Equations 3.25 and 3.29, and similarly $\tau_{13}^{I}$ and $\tau_{13}^{I I}$ from Equations 3.25 and 3.30 in closed from. Using Equations 3.27 and 3.28 , the remaining local strain components can be found.

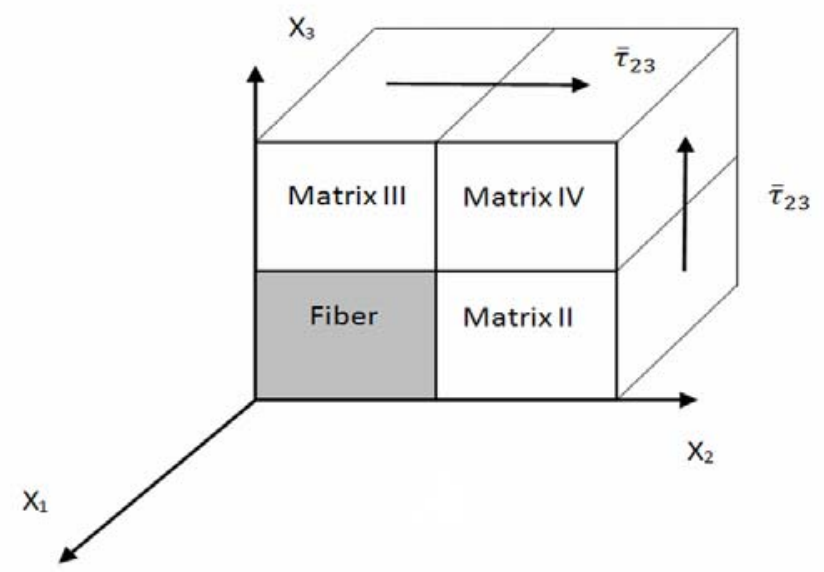

Figure 3-3: The RVE subject to shear loading

\subsection{Micromechanical Analysis of the Composite Strength}

Unlike the macromechanical approach where failure is controlled by the properties of the bulk composite material, in a micromechanical analysis, the failure of each of the constituents i.e. fiber and matrix has to be investigated separately. However, in many cases, the ultimate strengths of the fiber and matrix constituents are not readily available or are not always given by those measured from the bulk fiber or matrix materials (Aboudi, 1988). Therefore, good estimates of the ultimate strengths of the constituents are needed for the prediction of failure of a unidirectional composite material. These constituents' strengths can be obtained using micromechanical analysis. Since the micromechanical model is capable of calculating the local stresses $\sigma_{i j}^{(k)}$ i.e. the stresses in the fiber and matrix subcells from globally applied stresses $\bar{\sigma}_{i j}$ 
, it can be used to find the ultimate strengths of the fiber and matrix constituents from the knowledge of the allowable stresses or strengths of the unidirectional composite in the principal material directions i.e. in longitudinal, transverse and loadings. Based on the SUCM model, the globally applied stresses $\bar{\sigma}_{i j}$ can be related to the subcell stresses $\sigma_{i j}^{(k)}$ as follows:

$\{\sigma\}^{k}=[C]\{\bar{\sigma}\}$

It should be noted that the elements of $[C]$ are functions of the material property of the fiber and the matrix material. Based on this concept, if $X, Y$ and $S$ denote, respectively the axial, transverse and in-plane shearing strengths of the composite, $X_{f}$ (the longitudinal ultimate stress of the fiber), $Y_{m}$ (the tensile strength of the matrix), and $S_{m}$ (the shear strength of the matrix) can be calculated as follows:

$$
\begin{aligned}
& X_{f}=\alpha X \\
& Y_{m}=\lambda Y \\
& S_{m}=\gamma S
\end{aligned}
$$

where $\alpha, \lambda$ and $\gamma$ are defined as follows:

$$
\begin{aligned}
& \alpha=\frac{\sigma^{(I)} 11}{\bar{\sigma}_{11}} \\
& \lambda=\max _{k=I I I I I, I V} \frac{\sigma^{(k)}{ }_{22}}{\bar{\sigma}_{22}} \\
& \gamma=\max _{k=I I, I I, I V} \frac{\sigma^{(k)}{ }_{12}}{\bar{\sigma}_{12}}
\end{aligned}
$$

In the above Equation, $\bar{\sigma}$ is the global average applied stress and $\sigma^{(k)}$ is the stress generated in the kth subcell. In fact, $\alpha, \lambda$ and $\gamma$, which relate the local stresses to the global applied stress, 
depend only upon the material properties of the fiber and matrix materials and the volume fraction. 


\section{CHAPTER 4: THE EFFECT OF TEMPERATURE ON THE VISCOELASTIC RESPONSE OF POLYMER MATRIX COMPOSITES}

In this chapter, the thermo-mechanical viscoelastic response of a high temperature polymer matrix composite system made up of T650-35 graphite fibers embedded in PMR-15 resin is studied through the Simplified Unit Cell Micromechanical (SUCM) model within a temperature range of 250 to $300^{\circ} \mathrm{C}$ corresponding to aerospace engine applications. Using the experimental data of the creep behavior of thermostable PMR-15 polyimide, the micromechanical model is first calibrated to account for the effect of temperature. The resulting elastic and viscoelastic responses are compared with the existing experimental data. The validated model is then used to predict the behavior of the composite material under different combinations of thermal and mechanical loadings.

Polymers are generally classified as either Thermoreheologically Simple Materials (TSM) or Thermorheologically Complex Materials (TCM). In the former, the effect of temperature is incorporated only through a time-scale shift factor (Harper and Weitsman, 1985). This assumption is valid as long as the configuration of the polymer molecular structure and thus its elastic and long-term responses are not affected by the temperature. However, as the temperature of the composite increases, the polymer matrix becomes more compliant and the composite's matrix dominated constants (shear and transverse moduli) decrease drastically (Odegard and Kumosa, 2000). In such cases the overall time-dependent behavior of the composite is governed by the matrix viscoelasticity. Therefore, it is necessary that the polymer matrix in high temperature PMC's be considered as a Thermorheologically Complex Material (TCM). 
The nonlinear viscoelastic behavior of polymers has been studied mainly for particulate composites. Ozupek and Becker $(1992,1997)$ developed a constitutive model for viscoelastic materials to study the mechanical behavior of solid rocket propellants which are in fact, particulate composites with a lightly cross-linked elastomeric binder. Temperature effects and a large range of deformations result in a highly nonlinear response of the material. Later, Conga et al. (2001) presented the computational implementation of this model with damage using the finite element method. Ha and Schapery (1998) developed and experimentally verified a three-dimensional non-linear viscoelastic model for particle-filled rubbers including damage. It was assumed that damage led to transverse isotropy with the axis of isotropy coinciding with the local, instantaneous maximum principle stress direction for monotonic loading.

Few works have hitherto incorporated micromechanical models to study the nonlinear viscoelastic response of TCM polymer matrix composites at high temperatures. Hashin et al. (1987) modeled a unidirectional fiber composite with a TCM matrix by concentric cylinder assemblage to predict its thermo-viscoelastic response. Sadkin and Aboudi (1989) used the method of cell (MOC) to study the viscoelasic response of TCM resin matrix composites. The aforementioned models assume that the isotropic matrix is viscoelastic in shear only. Recently Muliana et al. (2008) derived a micromechanical model for TCM polymer matrix composites with a temperature range of $23^{\circ} \mathrm{C}$ to $65^{\circ} \mathrm{C}$.

From among different polymer matrix resins, the PMR-15 polyimide has been designed to operate under extreme thermomechanical loading of carbon/PMR-15 composites usable in 
military aeroengine applications within a temperature range of 250 to $300^{\circ} \mathrm{C}$ (Rupnowski et al., 2006). Odegard et al. (2000) measured the elastic properties of a unidirectional composite based on T650-35 graphite fibers embedded in a PMR-15 polyimide resin for a temperature range from 25 to $315^{\circ} \mathrm{C}$ experimentally. However, micromechanical modeling of the viscoelastic behavior of carbon/PMR-15 composites subject to high temperatures is still lacking. One important issue which must be taken into account for high temperature polymer materials is a phenomenon called chemical aging which refers to an irreversible change in the polymer chain/network through mechanisms such as cross-linking or chain scission. One example of chemical aging is oxidation which takes place when the material is exposed at elevated temperatures (close to the glass transition temperature) to oxygen-rich environments for long periods of time. Significant shrinkage strains are induced due to thermo-oxidation in the resin that can lead to the development of micromechanical damage in the material (Pochiraju et al., 2008).

\subsection{Effect of Temperature on the Modulus of the Polymer Matrix}

Fiber reinforced polymer matrix materials fall into the category of vicoelastic materials due to their polymer matrix constituent and this vicoelasticity effect of the polymer matrix becomes more pronounce as the temperature increases. Figure 4.1 schematically shows how the Young modulus of a typical polymer changes with temperature. As illustrated in the figure, four regions of viscoelastic behavior can be identified as the glassy, transition, rubbery and flow. Two important temperatures are extracted from the curve of figure 4.1: namely the glass transition temperature $T_{g}$ and the melt temperature $T_{m} . T_{g}$ is the temperature at which the polymer starts changing from a brittle or glassy state into a rubbery or soft state. 


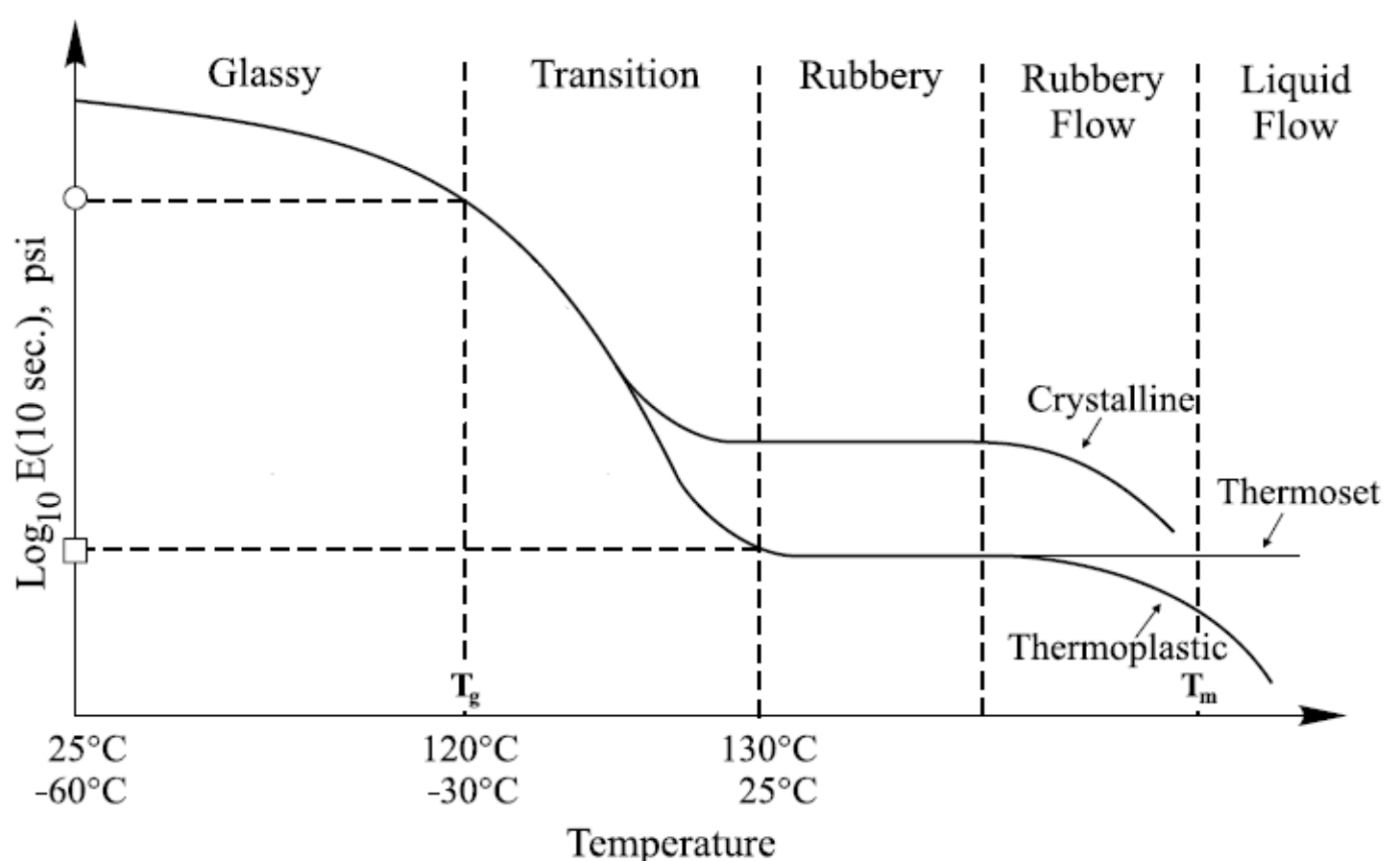

Figure 4-1: Variations in the Young modulus of a typical polymer with temperature (Brinson and Brinson, 2008)

\subsection{Time Temperature Superposition (TTS) Principle}

According to the time temperature superposition principle the viscoelastic behavior at one temperature can be related to that of another temperature by a change in time scale (Brinson and Brinson, 2008). Using TTS, one can characterize the viscoelastic properties of a material over a long time by obtaining data at different temperatures over a short period of time. This is done through creating the so called master curve by shifting the material property (modulus, compliance or Poisson's ratio) horizontally and vertically versus time on a log-log scale to a reference temperature. The resulting master curve is then used to show the change of that property over a long period of time which would have otherwise been impossible to obtain by doing experiment. Mathematically, this principle can be stated as:

$$
P\left(T_{1}, t\right)=P\left(T_{2}, \frac{t}{a(T)}\right) b(T)
$$


Where $T$ is the temperature, $P$ is the property under consideration and $a(T)$ and $b(T)$ denote the horizontal and vertical shift factor. Figure 4.2 shows schematically how the master curve is generated for the modulus of a viscoelastic material. If the temperature effects can be accounted for only through the shift factor $a(T)$, the material is called thermorheologically simple (TSM) otherwise it is classified as a thermorheologically complex material (TCM).

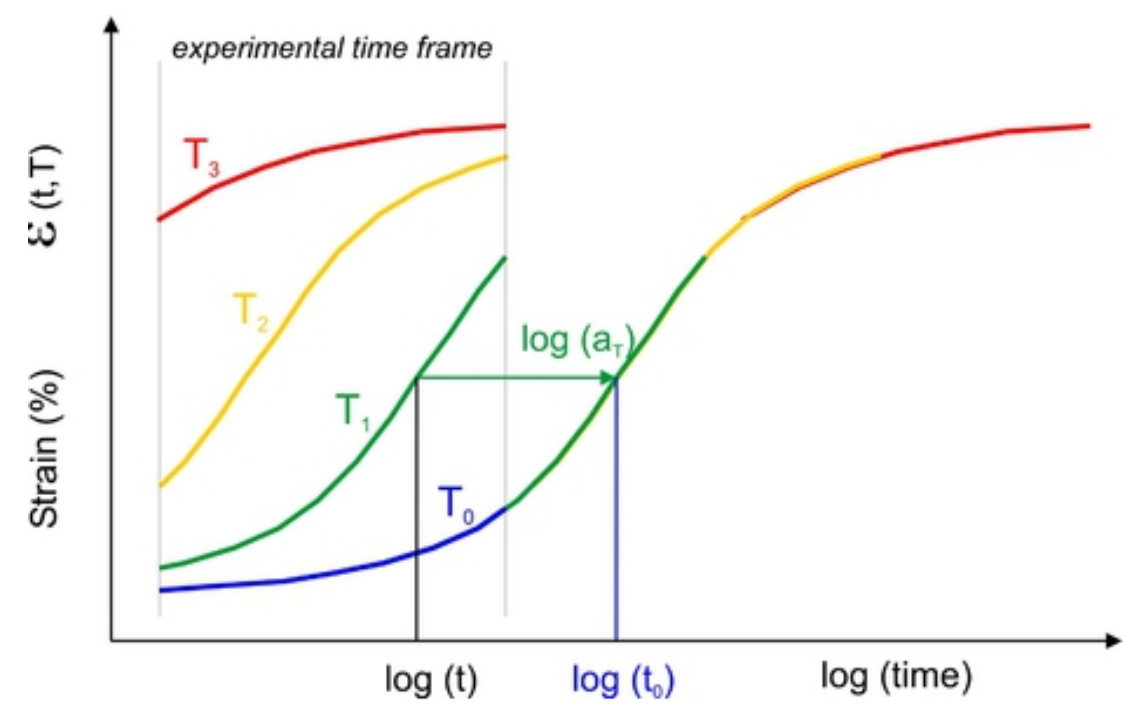

Figure 4-2: Schematic representation of how a master curve is generated

\subsection{The Viscoelastic Response of Thermo-Stable PMR-15}

As previously mentioned, the PMR-15 resin is used in composite materials under thermomechanical loading conditions in aerospace applications. The PMR-15 matrix is considered to be thermally stable up to about $300^{\circ} \mathrm{C}$ and the composite system is designed to operate in temperatures between 250 and $300^{\circ} \mathrm{C}$. Marais and Villoutreix (1998) studied the behavior of PMR-15 polyimide by performing tests under different conditions of stress and time. The loading time for each stress level was chosen as the mean time during which the material operates in real application in a military aircraft engine. The tests were carried out at 
four different temperatures to determine the maximum stress below which the material can be considered linear. The linear range ensures the integrity of the mechanical properties of the resin in case the composite system is subjected to repeated loading. The limit for the linear range was found to be between $0.6 \sigma_{r}$ for $250^{\circ} \mathrm{C}$ and $0.3 \sigma_{r}$ for $300^{\circ} \mathrm{C}$ where $\sigma_{r}$ is the static tensile failure stress measured to be $47 \mathrm{MPa}$ within the temperature range under consideration, which alludes to the thermal stability of PMR-15 polyimide. Based on the obtained experimental data, the creep compliance of PMR-15 within the linear range was assumed to take the following form (Marais and Villoutreix, 1998):

$$
J=\left(\frac{1}{E_{1}}\right)+\left(\frac{1}{E_{2}}\right) \times\left\{1-\exp \left(-\left(\frac{t}{\tau}\right)^{b}\right)\right\}+\left(\frac{t}{\eta}\right)
$$

Where $\tau$ denotes the retardation time, $b$ is the Kohlrausch coefficient which varies between 0 and 1 and $\eta$ is the viscosity. These parameters are adjusted based on the experimental data. The above-mentioned experimental data is used to calibrate the matrix subcells in the micromechainal model to account for the effect of temperaturce on the response of the composite material. The creep compliance in Equation 4.2 is seen to be composed of an elastic part and a time dependent viscous part. Now considering Equation 3.1, since the study of the PMR-15 resin is within the linear range, the following assumptions can be made: 1) The nonlinear coefficients i.e. $g_{0}, g_{1}, g_{2}$ and $a_{\sigma}$ are set equal to 1 . 2) The creep compliance can be defined based on the proportionality between the strain $\varepsilon$ and the stress $\sigma$. It can be observed that the matrix material response also consists of an elastic portion as well as a time dependent viscoelastic part. Hence, the calibration of the micromechanical model is carried 
out following the equivalence between Equations 3.1 and 4.1. $f_{1}(T)$ is thus curve fitted so as to account for the temperature dependence of the elastic response of the material. In other words, The function $f_{1}(T)$ is determined from measuring the initial creep response of the material, i.e. at $t=0$ at different temperatures.

$$
f_{1}(T)=0.0000079976442 T^{3}-0.00617976 T^{2}+1.595838520 T-135.9804979
$$

Table 4.1 lists the Prony series terms for PMR-15. The first attempt to calibrate the time dependent part is to use the time temperature shifting parameter $a(T)$, which for PMR-15 is defined as follows (Muliana and Sawant, 2009)[39]:

$$
a(T)=0.00001333 T^{3}-0.011 T^{2}+3.0087 T-272
$$

The time temperature shifting parameter $a(T)$ is calculated from the well-known Time Temperature Superposition Principle (TTSP) stating that the viscoelastic behavior at one temperature can be related to that of another temperature by a change in time scale. For thermorheologically simple material, the creep compliance at one temperature can be related to another temperature by applying the temperature shift factor. However, since PMR-15 is a thermorheologically complex material, the temperature dependence of the shift factor alone, as shown in Figure 4.3, cannot represent the experimental data properly, especially for higher temperatures $\left(285\right.$ and $\left.300^{\circ} \mathrm{C}\right)$. Therefore, a function $f_{2}(T)$ is also included to account for the thermorheologically complex behavior of the material. The temperature dependent constitutive equation is therefore written as: 


$$
\begin{aligned}
& \varepsilon(t)=f_{1}(T) D_{0} \sigma(t)+f_{2}(T)\left[\sum_{n=1}^{N} D_{n}\left(1-\frac{1-\exp \left(-\lambda_{n} \Delta \psi^{t}\right)}{\lambda_{n} \Delta \psi^{t}}\right)\right] \sigma(t) \\
& -g_{1} \sigma \sum_{n=1}^{N} D_{n}\left[\exp \left(-\lambda_{n} \Delta \psi^{t}\right) q_{n}{ }^{t-\Delta t}-\frac{1-\exp \left(-\lambda_{n} \Delta \psi^{t}\right)}{\lambda_{n} \Delta \psi^{t}} \sigma(t-\Delta t)\right]
\end{aligned}
$$

Where

$$
f_{2}(T)=-0.0000081523809 T^{3}-0.00739776 T^{2}-2.17207619 T+209.232857
$$

Table 4-1: Time-dependent properties of PMR-15 resin (Muliana and Sawant, 2009)

\begin{tabular}{|c|c|c|}
\hline$n$ & $\lambda_{n}$ & $D_{n}\left(\mathrm{MPa}^{-1}\right)$ \\
\hline 1 & 30 & $7.5 \mathrm{e}-6$ \\
\hline 2 & 10 & $7.0 \mathrm{e}-6$ \\
\hline 3 & 2 & $20.0 \mathrm{e}-6$ \\
\hline 4 & 1 & $10.0 \mathrm{e}-6$ \\
\hline 5 & 0.5 & $2.0 \mathrm{e}-6$ \\
\hline 6 & 0.1 & $19.0 \mathrm{e}-6$ \\
\hline 7 & 0.01 & $190.0 \mathrm{e}-6$ \\
\hline 8 & 0.001 & $850.0 \mathrm{e}-6$ \\
\hline \multicolumn{2}{|c|}{$\mathrm{v}=0.36$} \\
\hline
\end{tabular}

Figure 4.4 shows that a good fit to the experimental data is achieved for different temperatures after including $f_{2}(t)$. It can be seen that the maximum error for the highest temperature i.e. $300^{\circ} \mathrm{C}$, with respect to experimental data, drops to $3.5 \%$ from $20.9 \%$ before the introduction of $f_{2}(T)$ as shown in Figure 4.3. It should be noted that the functions obtained above are valid only within the specified temperature range. In order to expand the model to a temperature outside of this range, experiments need to be conducted either at that very temperature or at a lower temperature. The methodology proposed here is totally experiment-dependent which serves as a complement to the Time Temperature Superposition Principle (TTSP) to incorporate the effect of temperature into the viscoelastic behavior of the matrix material through the use of Schapery equation. 


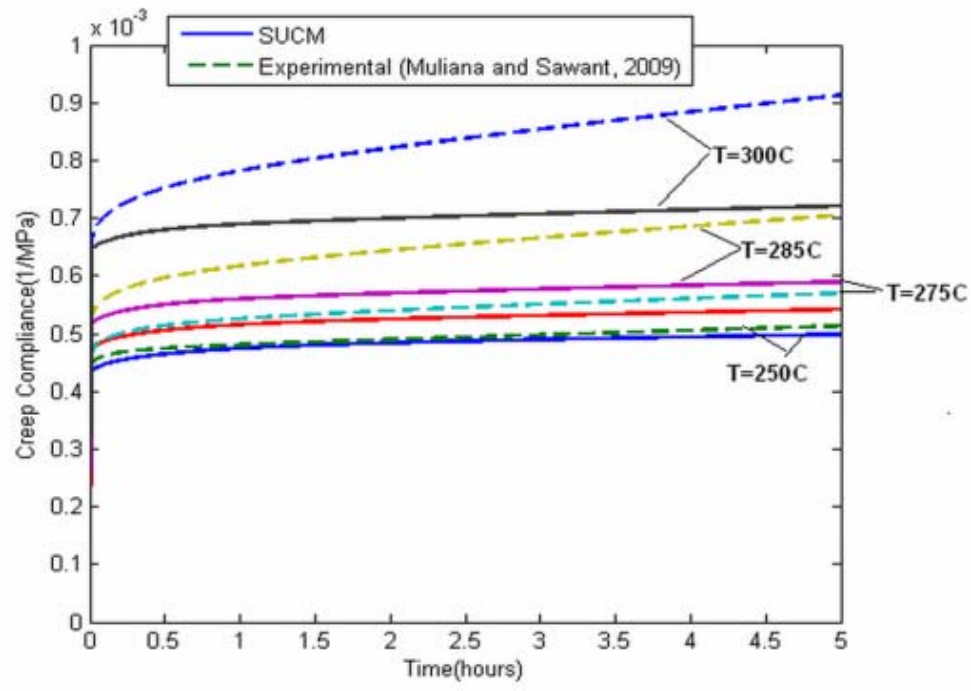

Figure 4-3: compliance for PMR-15 as a thermorheologically simple material at different temperatures

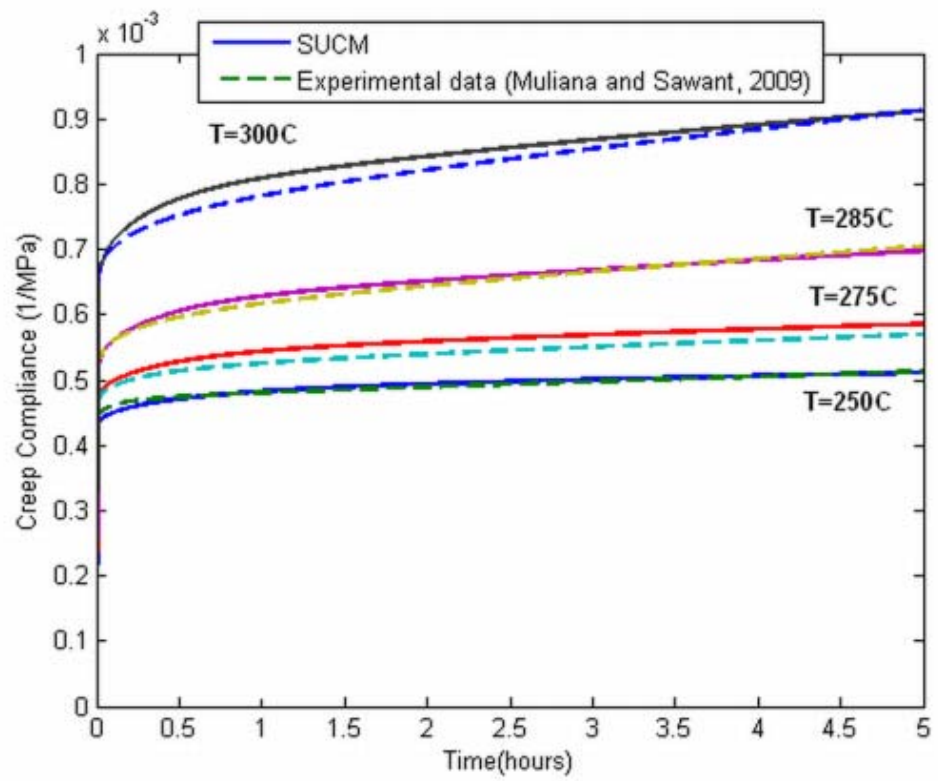

Figure 4-4: Creep compliance for PMR-15 as a thermorheologically complex material at different temperatures 


\subsection{Elastic Properties of Unidirectional T650-35/PMR-15 Composite as a Function of Temperature}

Odegard and Kumosa (2000) have measured the elastic properties of unidirectional T65035/PMR-15 composite experimentally. To further validate the present approach, the temperature dependent micromechanical model is used to calculate the elastic properties of unidirectional T650-35/PMR-15 composite with 56\% fiber volume fraction and the results are compared with available experimental data as well as with the Mori-Tanaka approach used by Rupnowski et al. (2006).

The elastic properties of T650-35 fibers are shown in Table 4.2. These properties are assumed to be temperature independent. As shown in Figure 4.5, the results are in reasonable agreement with the literature. It can be seen that the fiber dominated properties, i.e. the axial modulus and the longitudinal Poisson's ratio do not significantly change with temperature (Figures 4.5a and 4.5d), whereas the transverse and shear moduli which are dominated by the matrix properties degrade as the temperature increases (Figures $4.5 \mathrm{~b}$ and $4.5 \mathrm{c}$ ). In other words, the degradation of fiber dominated properties with temperature compared to that of the matrix dominated properties is not as significant. Regarding the scattered nature of the reported experimental data and thus considering the closer experimental data point to the calculated results, the mean absolute percentage errors (MAPE) for the transverse modulus and the shear modulus are calculated to be $8 \%$ and $18 \%$, respectively. Having established the validity of the present model, we now consider the response of the T650-35/PMR-15 composite system to thermomechanical loading. 
Table 4-2: Elastic properties for T650-35 graphite fiber(Rupnowski et al., 2006)

\begin{tabular}{|l|c|c|c|c|c|c|}
\hline Property & $E_{11}(\mathrm{GPa})$ & $E_{22}=E_{33}(\mathrm{GPa})$ & $G_{12}=G_{13}(\mathrm{GPa})$ & $G_{23}(\mathrm{GPa})$ & $\mathrm{v}_{12}=\mathrm{v}_{13}$ & $\mathrm{v}_{23}$ \\
\hline Value & 224 & 15.4 & 21.1 & 5.8 & 0.40 & 0.4 \\
\hline
\end{tabular}

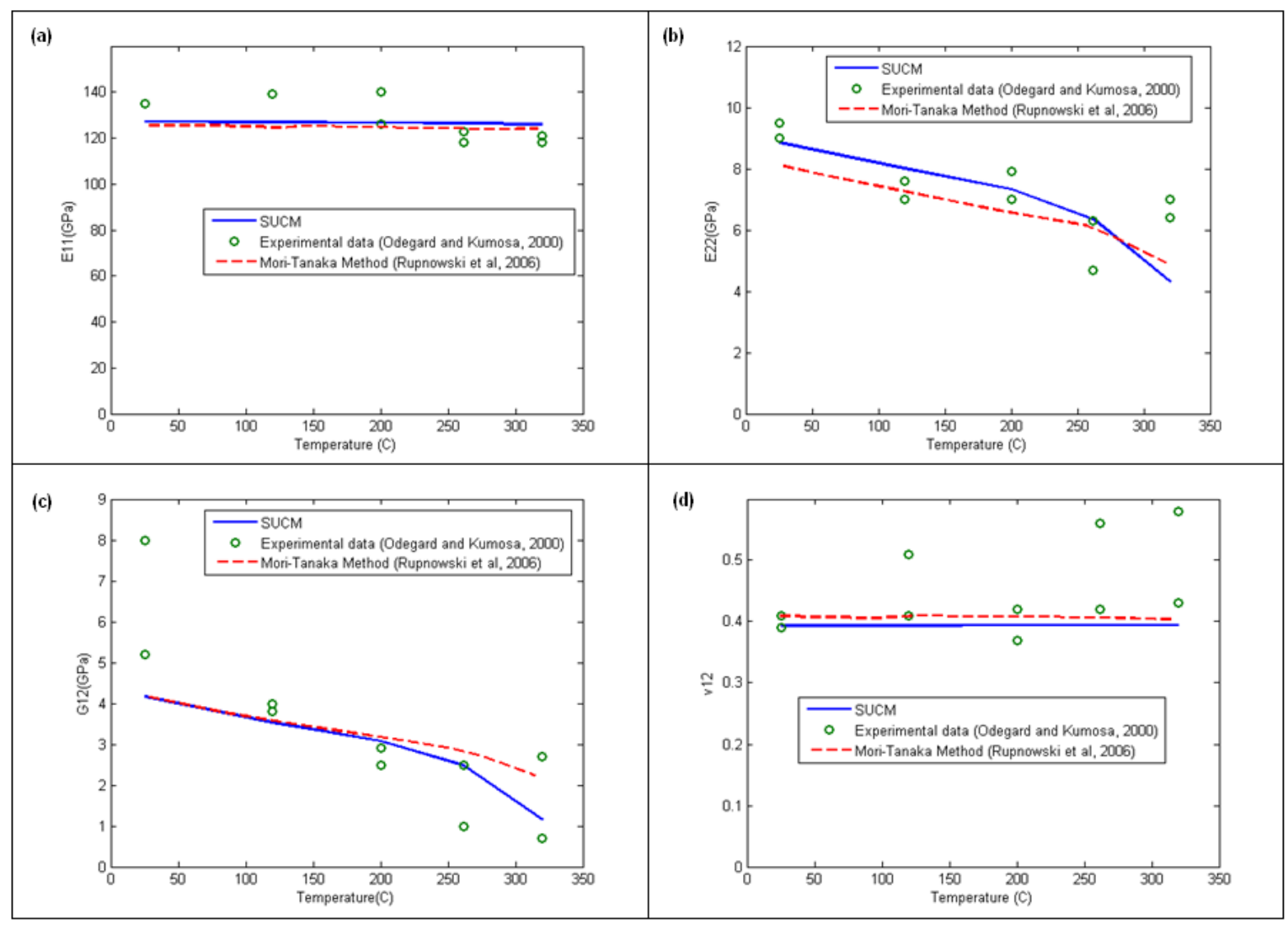

Figure 4-5: The elastic properties of unidirectional T650-35/PMR-15 composite: a) Axial modulus b) Transverse modulus c) Shear modulus d) Longitudinal Poisson's ratio

\subsection{Thermomechanical Response under a Linearly Increasing Stress}

The stress-strain responses of T650-35/PMR-15 under a monotonically increasing stress at the rate of $1 \mathrm{MPa} / \mathrm{sec}$ using the Simplified Unit Cell Micromechanical (SUCM) model are shown in Figures 4.6 and 4.7 for the transverse tension and longitudinal shear loads at different temperatures. It can be seen that both of these load cases are affected by temperature and that this effect becomes more pronounced as the load increases. The maximum difference 
between the values of axial and shear strains for the lowest and highest temperatures at the end of the loading time is estimated to be $34 \%$ and $45 \%$, respectively. These observations are in agreement with the experimental tests depicted in Figure 4.5 indicating the temperature dependence of matrix dominated properties of the composite. From a physical perspective, it can be deduced that increasing the temperature causes the viscosity of the fluid phase of the viscoelastic material to decrease such that the external load will thus be supported only by the solid phase which as a result will decrease the modulus.

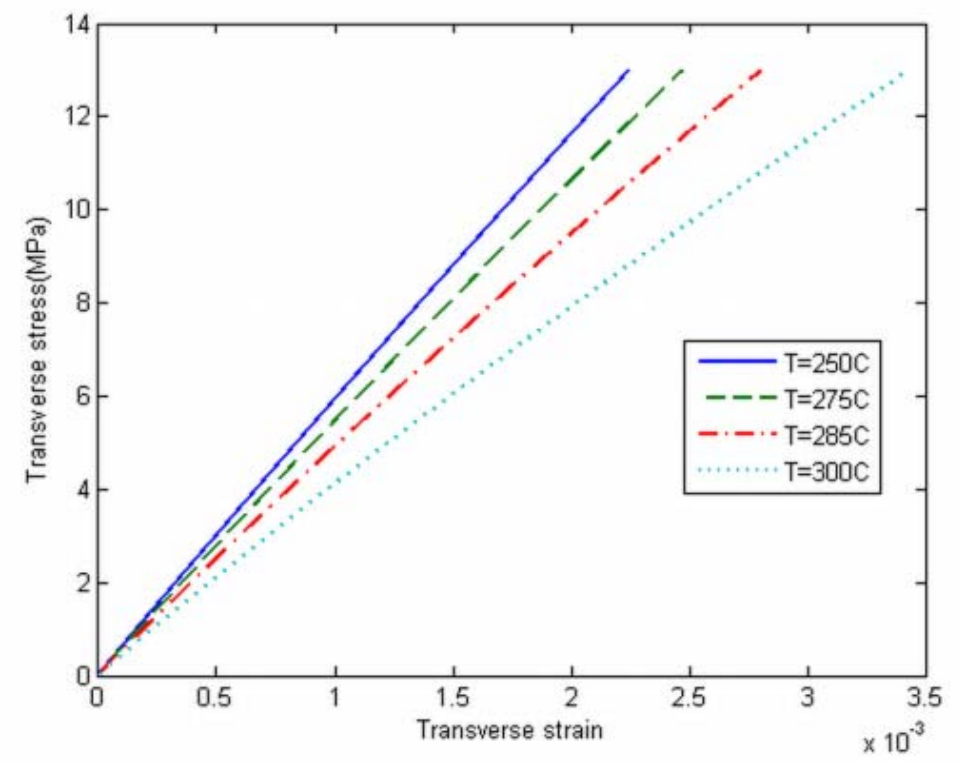

Figure 4-6: Transverse stress vs. strain at different temperatures for unidirectional T650-35/PMR-15 composite 


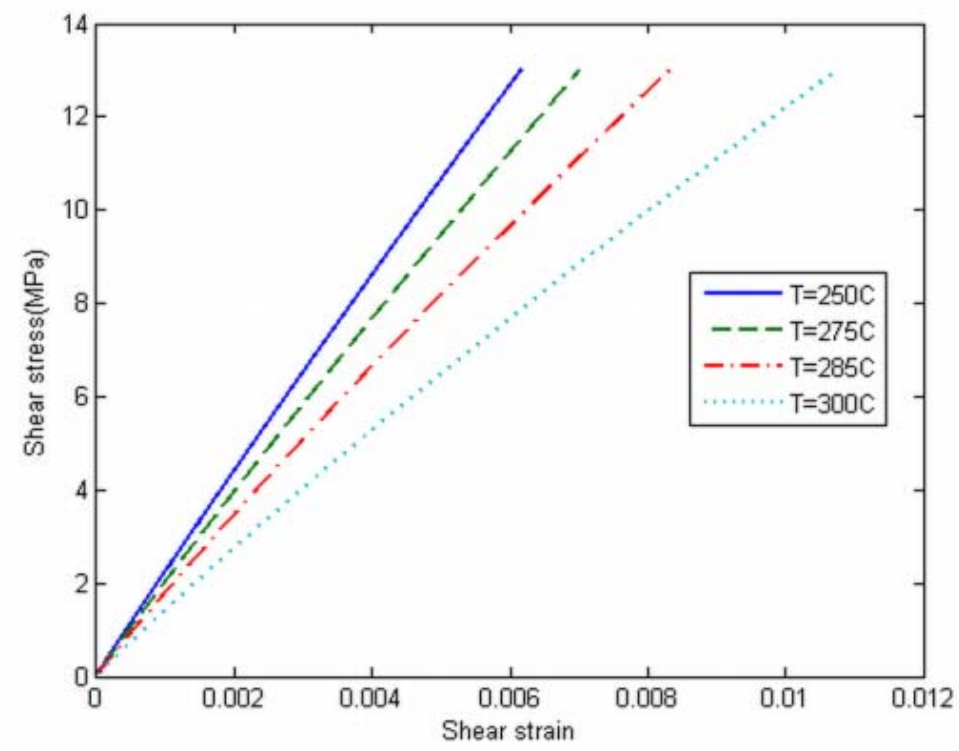

Figure 4-7: Shear stress vs. strain at different temperatures for unidirectional T650-35/PMR-15 composite

\subsection{Thermomechanical Creep Loading}

Figure 4.8 shows the response of the composite system to a constant transverse stress of 1 $\mathrm{MPa}$ at four different temperatures. Figure 4.9 shows the creep strain response under a linear temperature ramp. In both Figures 4.8 and 4.9 the acceleration of the creep strain and softening of the initial and time-dependent portions of the composite response with increasing temperature are apparent. In particular, as Figure 4.9 shows, the change in the material response for higher temperatures, i.e. above $275^{\circ} \mathrm{C}$ is observed to be more abrupt. 


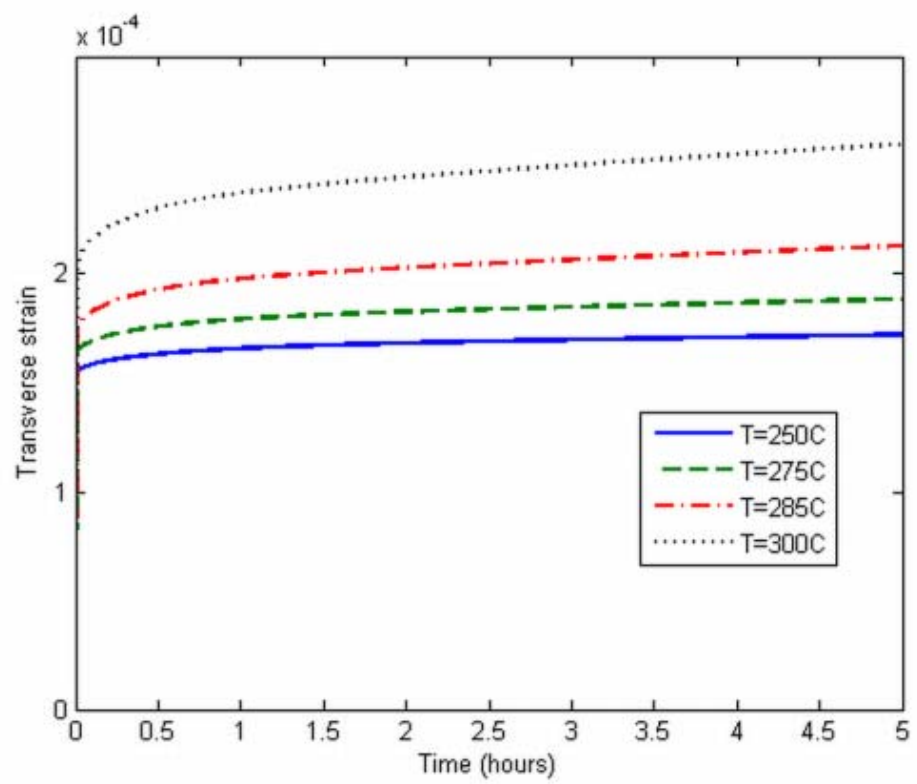

Figure 4-8: Transverse creep strain of unidirectional T650-35/PMR-15 composite at different temperatures $(\sigma=1 \mathrm{MPa})$

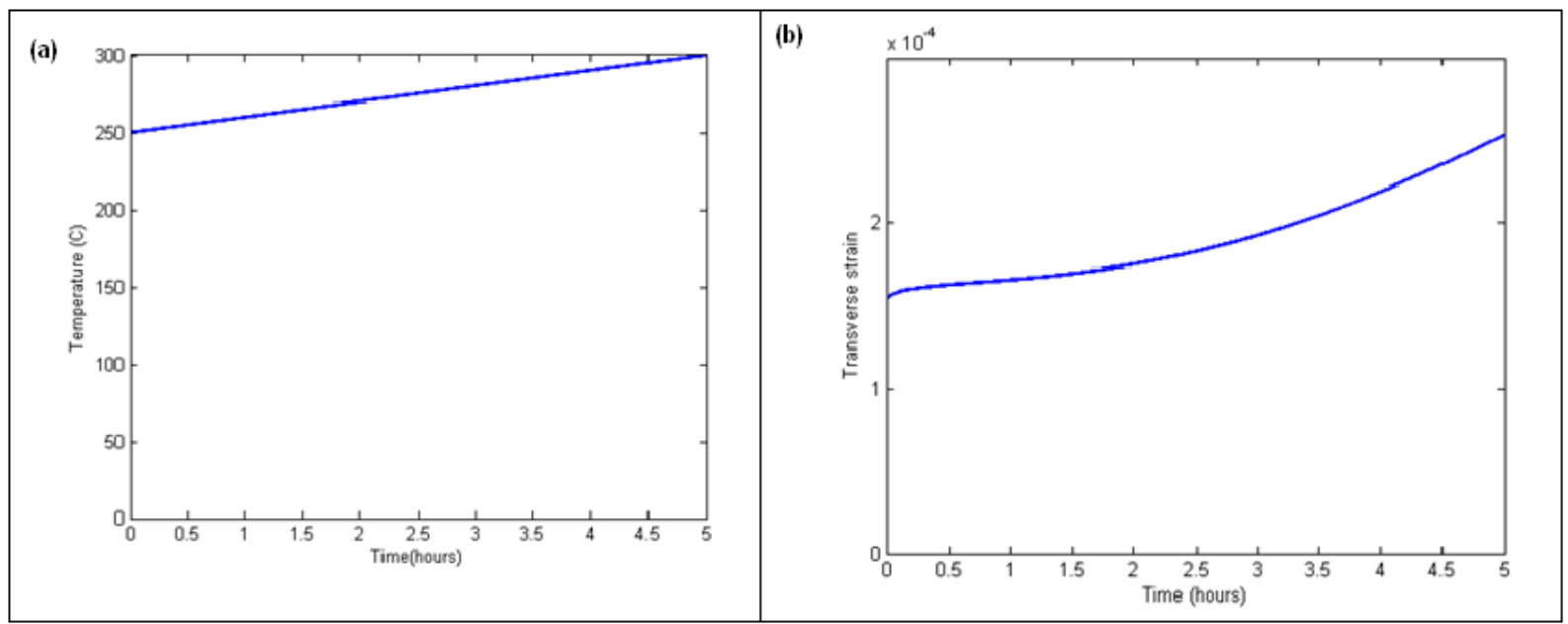

Figure 4-9: a) Temperature ramp b) Transverse creep strain of unidirectional T650-35/PMR-15 composite under linear temperature ramp $(\sigma=1 \mathrm{MPa})$

The next step is to predict the response of the composite to a cyclic temperature change.

Since this kind of composite is for potential applications at temperatures between 250 and $300^{\circ} \mathrm{C}$, the temperature cycle is defined as shown in Figure 4.10a with extremes of 250 and $300^{\circ} \mathrm{C}$ within a period of 5 hours based on the average duration of loading of a real military aircraft. The transverse normal strain produced by the combination of creep and cyclic 
temperature loading is shown in Figure $4.10 \mathrm{~b}$. The total strain is seen to increase due to the accumulation of creep strain during each cycle. However, this increase becomes less pronounced from cycle to cycle as the number of the cycles increases. In addition, it can be seen that the rate of the strain change between $250^{\circ} \mathrm{C}$ and $275^{\circ} \mathrm{C}$ i.e. within the second half of each cycle is much less compared to the first half. This is attributed to functions $f_{1}(T)$ and $f_{2}(T)$ described in Equations 4.3 and 4.6, respectively to fit the experimental data. Figure 4.11 shows these two functions versus temperature. As the figure shows, the rate of the change of these two functions between the 250 and $275^{\circ} \mathrm{C}$ is much lower than between 275 and $300^{\circ} \mathrm{C}$.
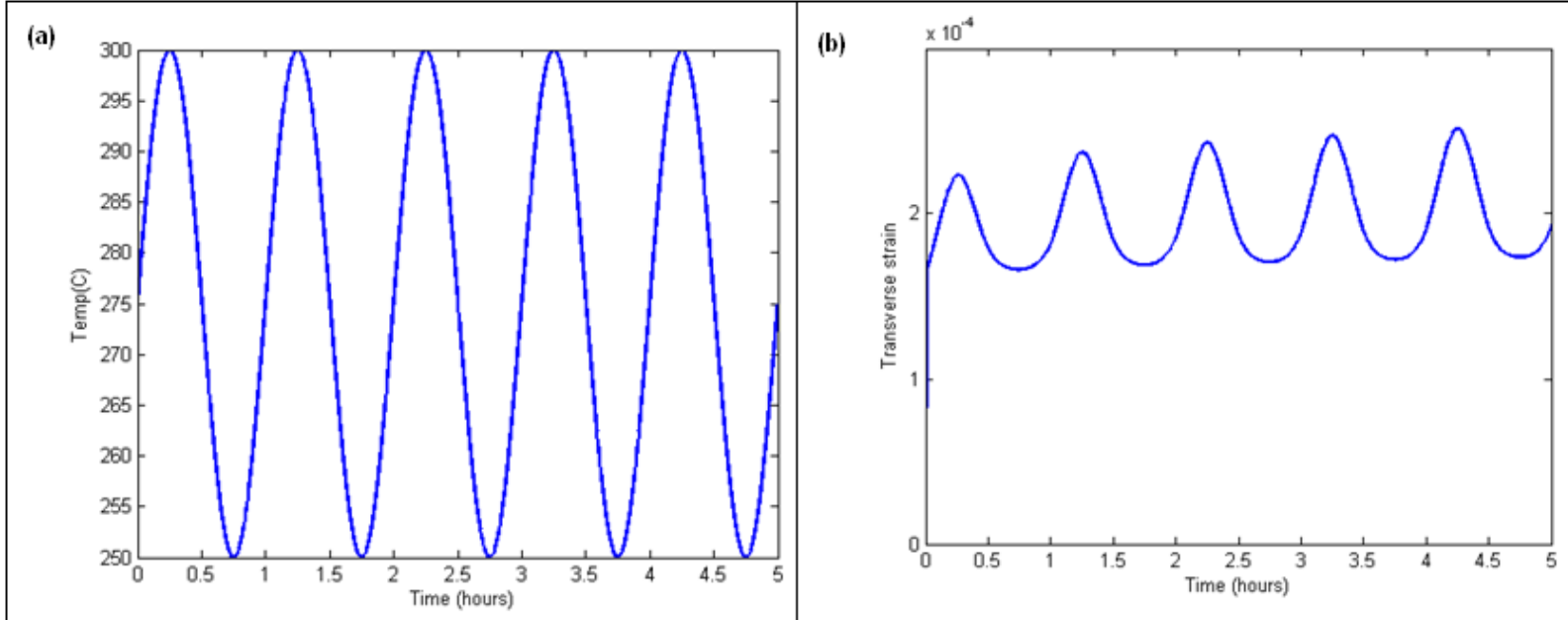

Figure 4-10: a) Temperature cycle b) Transverse creep strain of unidirectional T650-35/PMR-15 composite under cyclic temperature loading $(\sigma=1 \mathrm{MPa})$ 
(a)

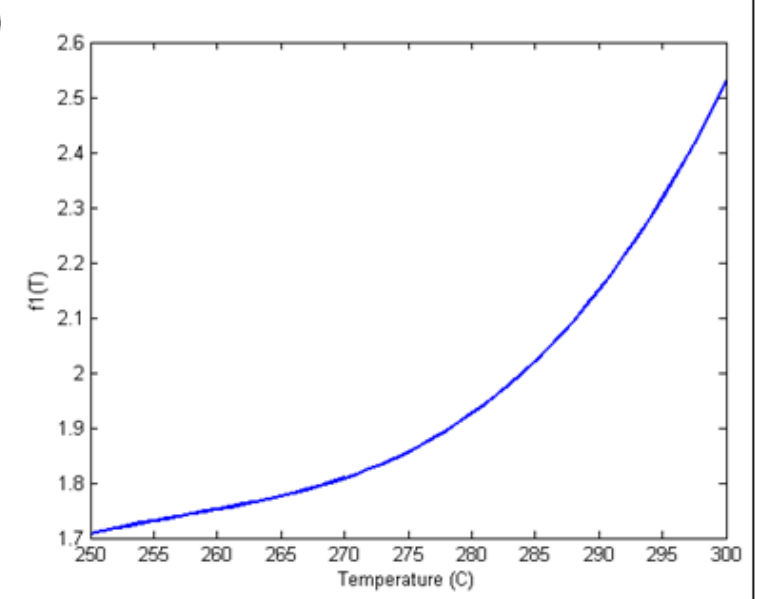

(b)

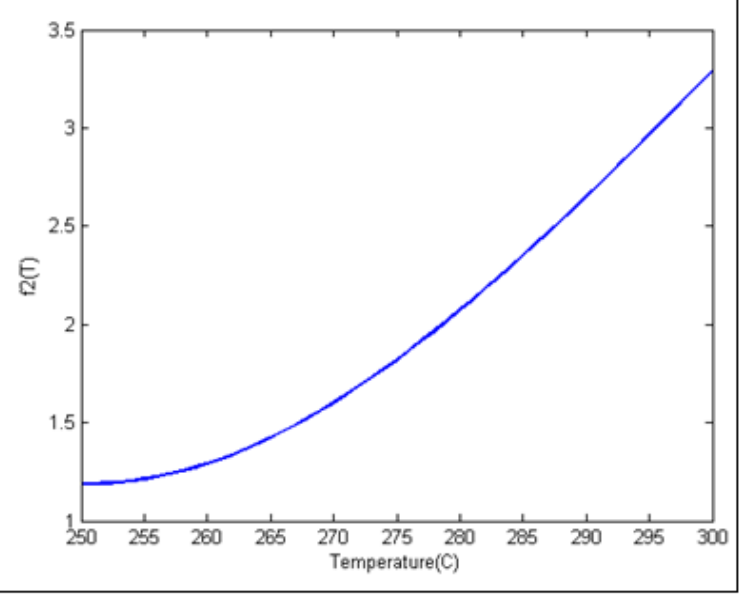

Figure 4-11: Temperature dependent functions a) $f_{1}(T)$ b) $f_{2}(T)$

Figure 4.12 depicts isochronous stress-strain curves for different temperatures after 5 hours. It is observed that as the stress increases, the curves diverge and that the increase of temperature gives rise to the softening of the material and consequently the creep strain.

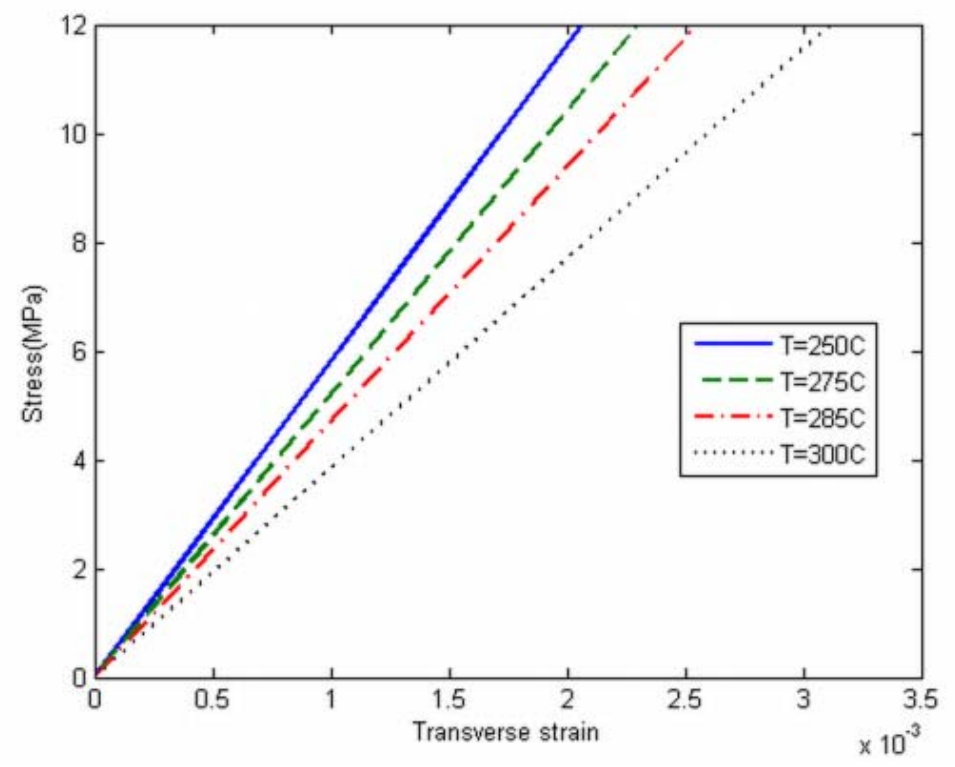

Figure 4-12: Isochronous stress-strain curves of T650-35/PMR-15 composite for different temperatures after 5 hours 


\subsection{Elastic versus Viscoelastic Response}

In this section, the T650-35/PMR-15 is subjected to a monotonically increasing transverse normal stress and the response of the composite material is studied with and without considering the time dependent portion of Equation 4.5. Figure 4.13 shows the difference between the resulting strains versus time at different temperatures. It can be seen that as the temperature increases, so does the difference between the elastic and viscoelastic response indicating the prominence of viscoelasticity with increasing temperatures. This increase becomes most noticeable for the highest temperature i.e. $\mathrm{T}=300^{\circ} \mathrm{C}$. This, in fact, corresponds to figure 4.4 where the creep compliance increases at a much higher rate with time for this temperature.

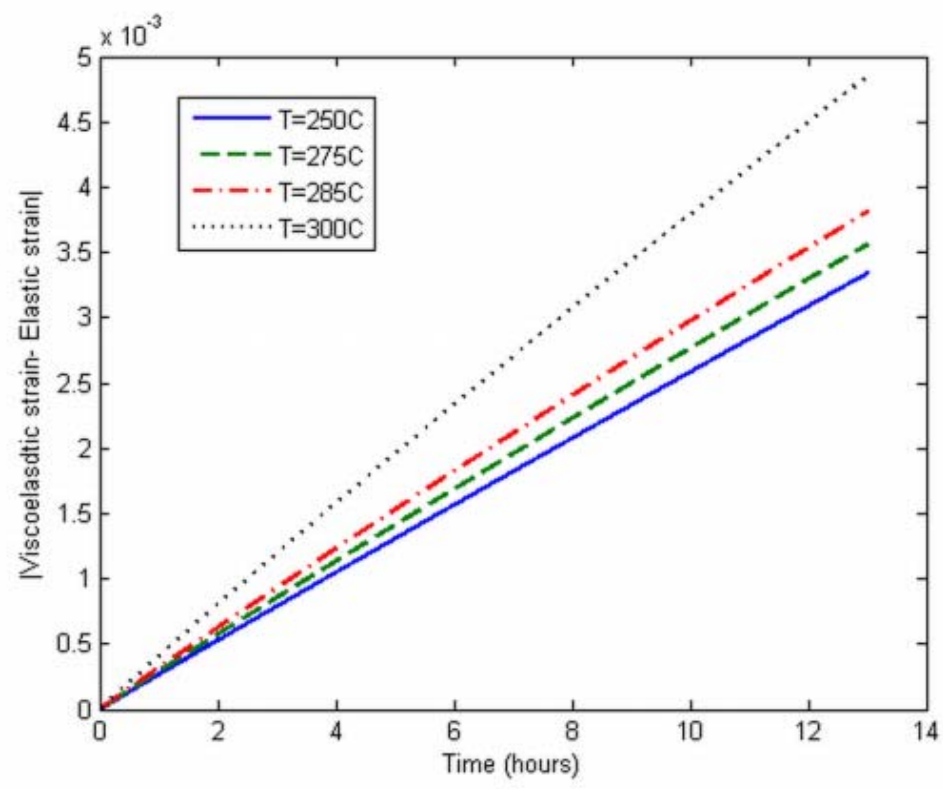

Figure 4-13: Difference between the elastic and viscoelastic response of T650-35/PMR-15 composite versus time at different temperatures. 


\subsection{Creep Recovery and Reloading}

Figure 4.14 shows the strain evolution upon loading-unloading and reloading for the T65035/PMR15 composite for different stress levels at $\mathrm{T}=250^{\circ} \mathrm{C}$ where $\sigma_{r}$ is the static tensile failure stress of the PMR-15 matrix material. It can be seen that after the material is unloaded and allowed to relax for 2 hours, the strain does not recover completely. Another important point to be noted is that the instantaneous compliance of the material to the load as well as the residual strains increase with increasing load. Figure 4.15 shows how temperature affects the loading-unloading and reloading. The residual stress upon unloading is seen to have increased with temperature. Moreover, since the applied stress is within the linear range i.e. $0.3 \sigma_{r}$, the maximum strain level is attained upon reloading the specimen.

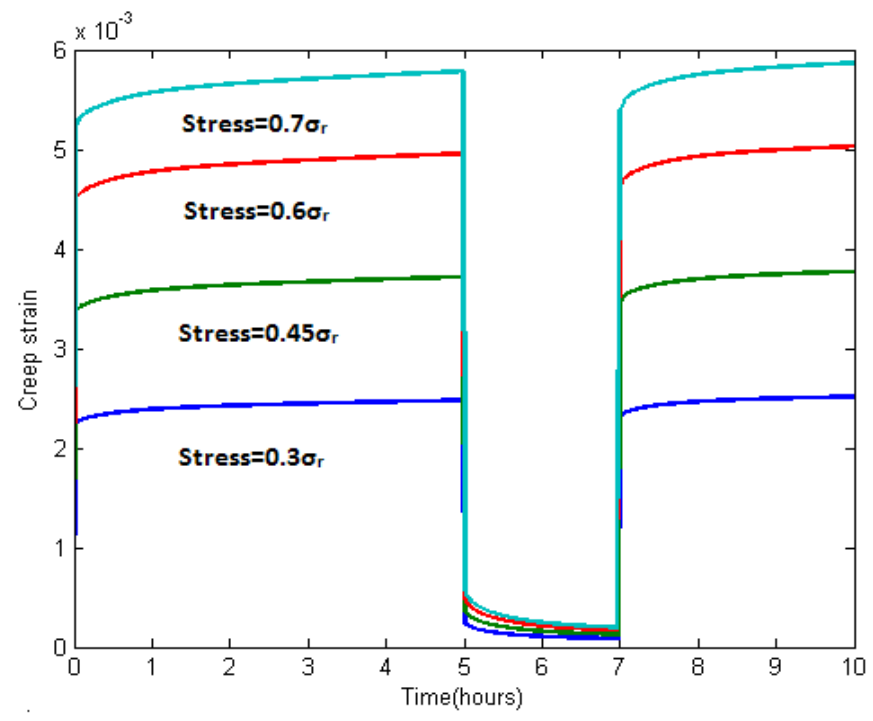

Figure 4-14: Strain evolution upon loading-unloading and reloading for T650-35/PMR15 composite for different stress levels at $\mathrm{T}=250^{\circ} \mathrm{C}$ 


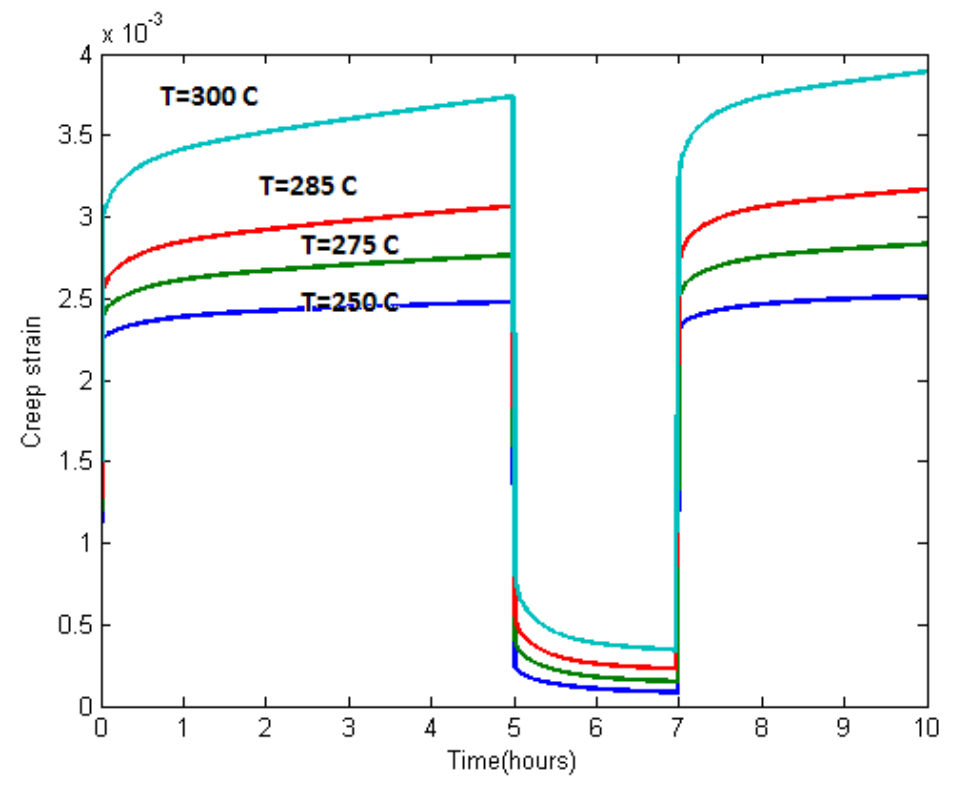

Figure 4-15: Strain evolution upon loading-unloading and reloading for T650-35/PMR15 composite at different temperatures $\left(\sigma=0.3 \sigma_{\mathrm{r}}\right)$

\subsection{Conclusion}

A micromechanical model, based on the assumptions of the Simplified Unit Cell Micromechanical (SUCM) model is utilized to predict the thermo-mechanical viscoelastic response of a high temperature polymer matrix composite system made up of T650-35 graphite fibers embedded in PMR-15 within a temperature range of 250 to $300^{\circ} \mathrm{C}$, corresponding to military aerospace engine applications. Current works on modeling temperature dependent viscoelastic behavior of polymer matrix composites are mainly based on the assumption of thermorheologically simple material. However, through the present approach, the matrix is modeled as a thermorheologically complex material.

The results are found to be in good agreement with the existing experimental data. The stress-strain response of the composite material under a monotonically increasing stress is seen to be affected by temperature in transverse and shear loadings which are dominated by 
matrix properties. When subject to creep loading, the acceleration of the creep strain and softening of the initial and time-dependent portions of the composite response with increasing temperature are apparent in particular, for temperatures above $275^{\circ} \mathrm{C}$ where the change in material response is more noticeable. Under cyclic creep loading, the total strain is seen to increase due to the accumulation of creep strain during each cycle although the rate of this increase drops from cycle to cycle as the number of the cycles increases. In general, the effect of temperature is observed to be more pronounced for temperatures higher than $275^{\circ}$ C. For instance, in case of cyclic loading, the rate of the strain change between $250^{\circ} \mathrm{C}$ and $275^{\circ}$ C i.e. within the second half of each cycle is much less compared to the first half. Through modeling the polymer resin as a thermorheologically complex material, the effect of temperature on the elastic and viscoelastic of the composite system can be individually investigated, which clearly demonstrates the importance of the time-dependent viscoelastic portion of the material response as the temperature increases. 


\section{CHAPTER 5: A MICROMECHANICAL APPROACH FOR THE PREDICTION OF THE TIME-DEPENDENT FAILURE OF HIGH TEMPAERATURE POLYMER MATRIX COMPOSITES}

In this chapter, the micromechanical approach is utilized to study the time dependent failure of unidirectional polymer matrix composites. Research studies conducted so far on the creep failure of polymer matrix composites are mainly based on macromechanics which predict the failure of composite as a bulk material. However, matrix time dependent failure due to creep is a localized phenomenon which demands a sufficiently accurate stress analysis of a representative volume elemen, where the composite cannot be treated as homogeneous. That's why micromechanical analysis gives a far better insight into the failure mechanisms of the composite materials. In fact, through micromechanics, the modes of failure in fiber and matrix can be separately identified which helps modify the stiffnesses of the constituents accordingly. Furthermore, since at high temperatures, the general behavior of the polymer matrix composite is governed by matrix viscoelasticity, the need for micromechanical analysis becomes more crucial. The creep failure criterion is expressed in terms of the creep failure functions of the viscoelastic matrix material assuming that the creep failure of the composite material is only due to the failure of the epoxy matrix. The main advantage of the present method is that it makes use of the micromechanical model to obtain the creep failure functions of the matrix material from the knowledge of the creep behavior of the bulk composite material in only transverse and shear loadings. Upon the calculation of these creep functions, the creep failure behavior of the composite for any off-axis degree can be predicted without the need for carrying out any experiments, which is of great benefit particularly at high temperatures where experimentation poses a lot of challenges. The composite material 
used in the study is T300/934 which is suitable for service at high temperatures in aerospace applications.

\subsection{Composite Creep Failure}

Unlike macromechanics where failure is controlled by the properties of the bulk composite material, the micromechanical modeling of a composite material makes it possible to investigate the failure modes of the constituent materials separately. However, in many cases, the ultimate strengths of the fiber and matrix constituents are not readily available or are not always given by those measured from the bulk fiber or matrix materials. For instance, in the case of a weak interface between fiber and matrix, the ultimate strengths of the matrix need to be modified in order to account for those weaker interfaces. Based on the concept explained in section 3.5 , the creep strengths of the matrix material can be calculated from the knowledge of the creep strength of the composite material in transverse and shear loadings by calculating $\max \left(\sigma_{22}^{k}\right)$ and $\max \left(\sigma_{12}^{k}\right)$, respectively.

Once these creep strengths are determined, the creep life of the unidirectional composite material at any off-axis degree can be obtained from testing of the composite in only transverse and shear loading which eliminates the need for any further experimentation. The creep failure criterion for the unidirectional composite is thus expressed as follows:

$\left[\frac{\sigma^{(k)}{ }_{22}}{Y_{\text {mcreep }}}\right]^{2}+\left[\frac{\sigma^{(k)}{ }_{12}}{S_{\text {mcreep }}}\right]^{2}=1$

where $k=I I, I I I, I V$, i.e. the matrix subcells. Unlike the static loading, $Y_{m c r e e p}$ and $S_{m c r e e p}$ are the creep failure strengths of the matrix material which are functions of time and temperature. The failure is assumed to have occurred once one the subcells fails the forgoing criterion. The 
major assumption of the present approach is that creep failure of the composite material is only due to the failure of the polymer matrix.

\subsection{Long Term Behavior of T300/934 Composite Systems}

One of the most important applications of composite materials in general, and high temperature PMC's in particular, is in the aerospace industry. Nowhere else, is there such a critical need for high performance of light weight material under severe conditions. T300/934 is one of the polymer matrix composite materials suitable for service at high temperatures in aerospace applications. In fact, this graphite epoxy fiber reinforced composite system is one of the few designated as "space-approved" by NASA. These fibers are produced by carbonizing high modulus organic precursors (Poly-Acrylonitrile, PAN) at high temperatures in an inert atmosphere. The epoxy resin is a thermosetting polymer binder that cures at $3500 \mathrm{~F}$. Table 5.1 shows the material properties of the fiber and matrix materials for this composite system.

Table 5-1: Glass and epoxy elastic material properties (Hiel, 1983)

\begin{tabular}{|l|c|c|c|c|c|}
\hline & $E_{11}(\mathrm{GPa})$ & $E_{22}(\mathrm{GPa})$ & $G_{12}(\mathrm{GPa})$ & $v_{12}$ & $v_{23}$ \\
\hline Graphite fiber & 200 & 13 & 44 & 0.39 & 0.40 \\
\hline Epoxy matrix & 4.1 & 4.1 & 4.1 & 0.35 & 0.35 \\
\hline
\end{tabular}

Hiel et al. (1983) conducted creep tests on $10^{\circ}$ off-axis T300/934 composites under shear loading at four different temperatures: $119^{\circ} \mathrm{C}, 148^{\circ} \mathrm{C}, 160^{\circ} \mathrm{C}$ and $168^{\circ} \mathrm{C}$. The average fiber volume fraction of the coupon tests was $60 \%$. Shear compliance master curve was created using the time-stress-superposition principal (TSSP) with the reference temperature of $119^{\circ} \mathrm{C}$. At each temperature, the experimental creep data was reported for different stress levels ranging from 1 to $5 \mathrm{ksi}$. Based on this data, the temperature shift factor $a(T)$ is calibrated as follows: 


$$
a(T)=-(2.4 e-7) T^{3}+0.00018 T^{2}-0.048 T+4.5
$$

The coefficients of the six-term prony series $\lambda_{n}$, as well as the nonlinear coefficients of the Schapery equation i.e. $g_{0}, g_{1}, g_{2}$ and $a_{\sigma}$ are taken from HajAli (2004). The SUCM is then employed to predict the creep strain of the composite for different stress levels for the reference temperature for $160^{\circ} \mathrm{C}$. This particular temperature was chosen since the creep failure of the composite material is investigated and compared with the experimental data at this temperature. Figure 5.1 shows the creep compliance of the material at $\mathrm{T}=160^{\circ} \mathrm{C}$. Good agreement is seen between the micromechanical model predictions and experimental results.

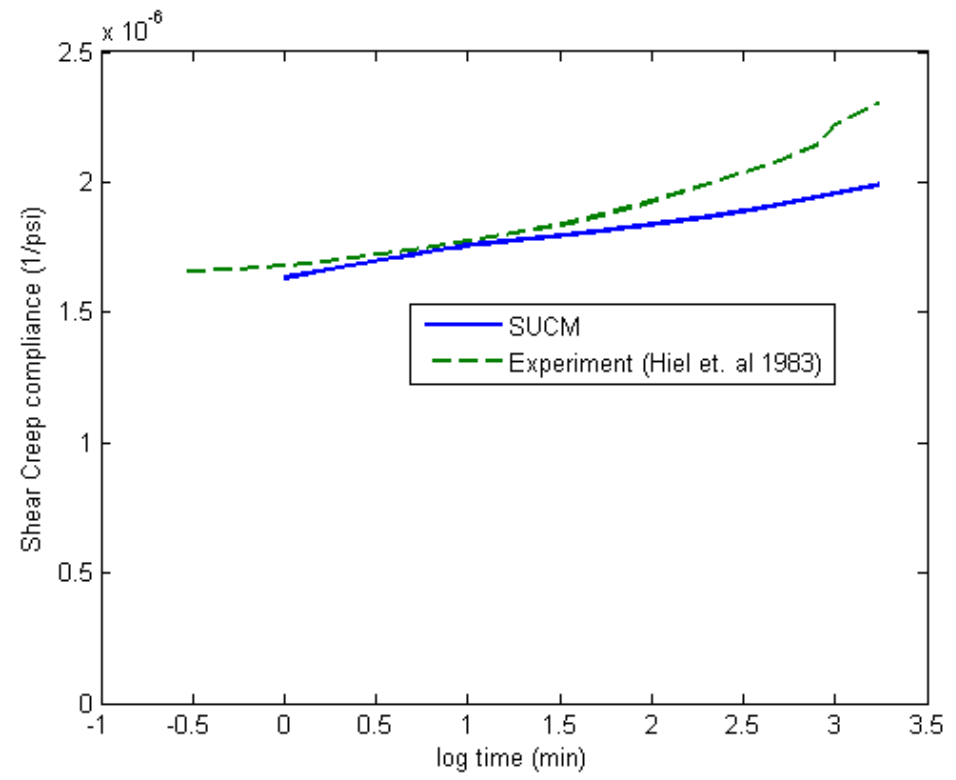

Figure 5-1: Shear creep compliance for $10^{\circ}$ off-axis $\mathrm{T} 300 / 934$ composite at $\mathrm{T}=160^{\circ} \mathrm{C}$

\subsection{Creep Failure of T300/934 Composites}

Figure 5.2 shows the creep failure time for different levels of stress of unidirectional T300/934 laminates in transverse and shear loading at $\mathrm{T}=160^{\circ} \mathrm{C}$, obtained by Dillard (1981). The micromechanical model uses this data as global loads to obtain the creep failure strengths of the matrix material as local stresses in the matrix subcells as functions of time. Using the data 
from figure 5.2, the following functions of time for the creep failure strengths of the matrix material are interpolated.

$$
\begin{aligned}
& Y_{\text {mcreep }}=34.18772+7.87171 \exp (-t / 1.67841)+9.04537 \exp (-t / 157.09826) \\
& +8.26342 \exp (-t / 10882.89576) \\
& S_{\text {mcreep }}=22.05621+5.90482 \exp (-t / 102.89117)+7.00561 \exp (-t / .62969) \\
& +6.78966 \exp (-t / 13023.39272)
\end{aligned}
$$

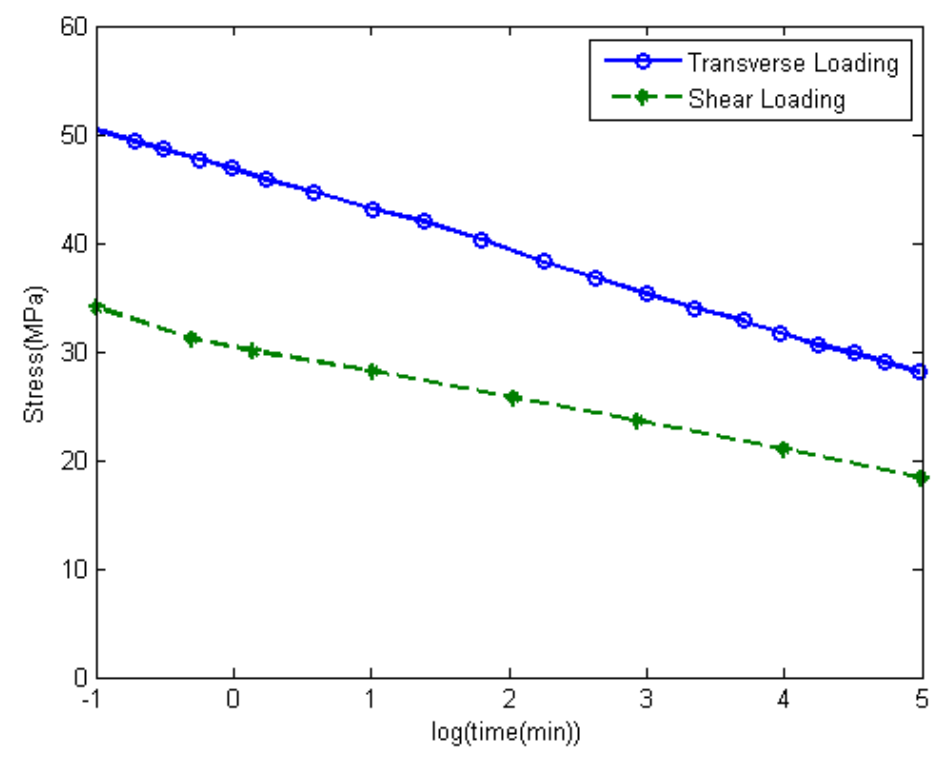

Figure 5-2: Creep Failure response of T300/934 composite in transverse and shear loading at $\mathrm{T}=160^{\circ} \mathrm{C}$ (Dillard, 1981)

The above- acquired functions are used in the creep failure criterion of Equation 5.1 which can then be used to find the creep failure time for any arbitrary off-axis angle. Figures 5.3-5.6 show the creep rupture predictions for different off-axis unidirectional laminates compared with the experimental results obtained by Brinson et. al (1981). As can be seen, there is reasonable agreement between experiment and predictions considering the inherent scatter associated with composite testing. 


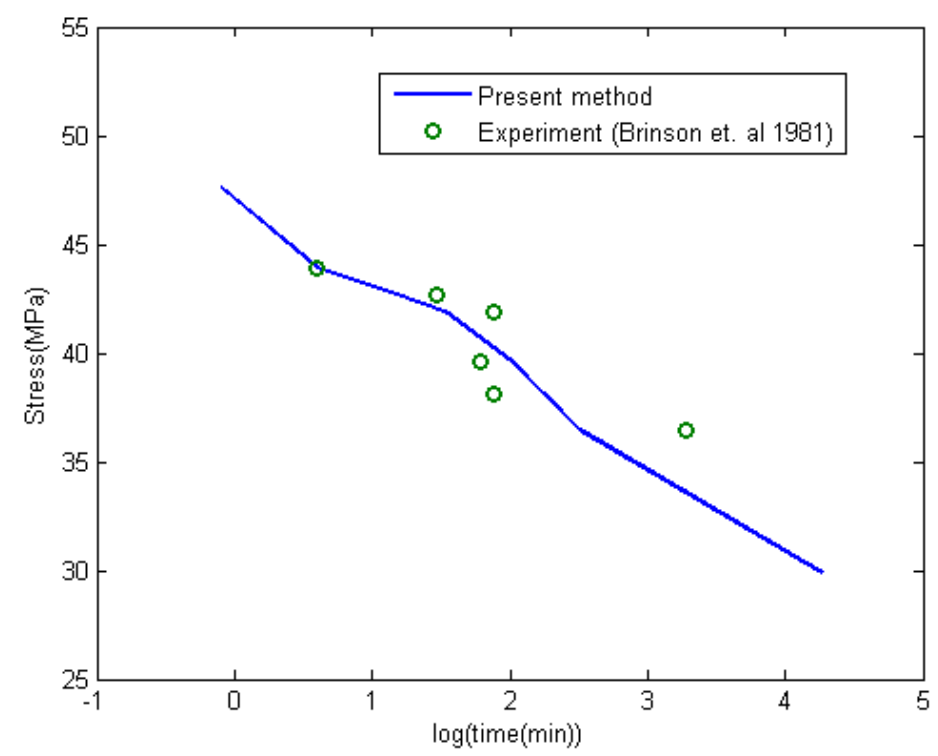

Figure 5-3: Creep failure time for $90^{\circ}$ off-axis $\mathrm{T} 300 / 934$ composite at $\mathrm{T}=160^{\circ} \mathrm{C}$

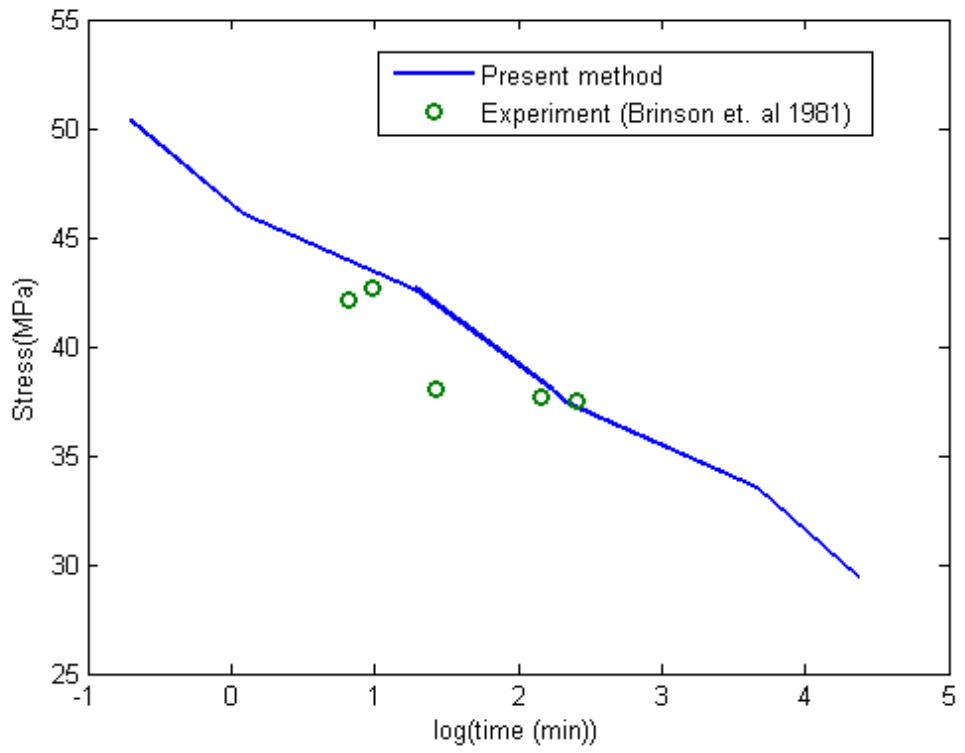

Figure 5-4: Creep failure time for $60^{\circ}$ off-axis $\mathrm{T} 300 / 934$ composite at $\mathrm{T}=160^{\circ} \mathrm{C}$ 


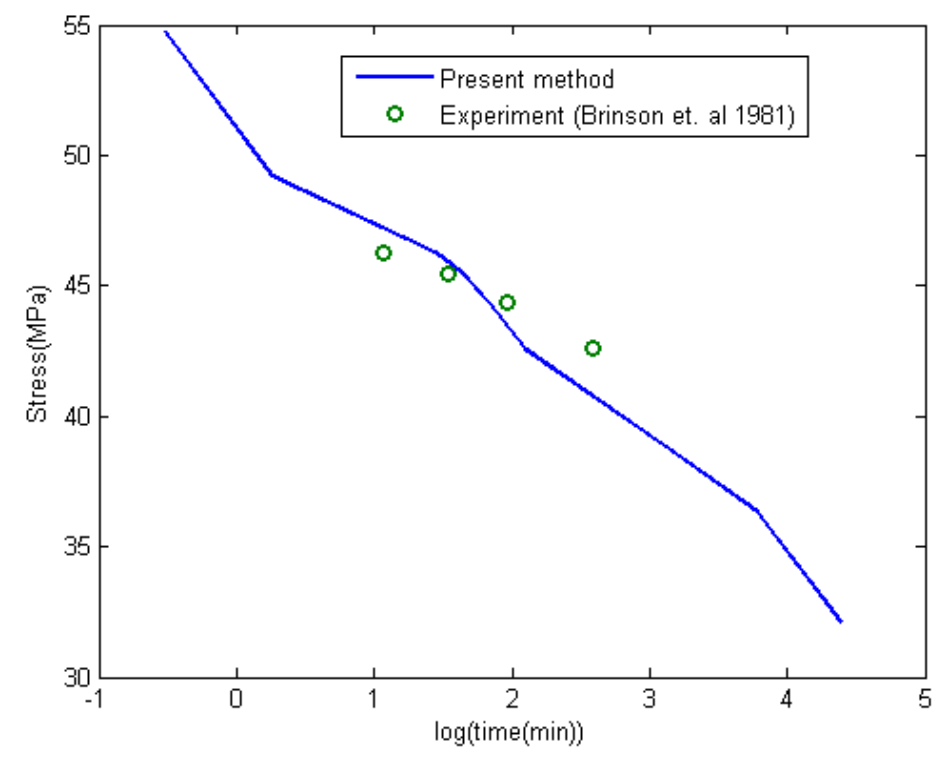

Figure 5-5: Creep failure time for $45^{\circ}$ off-axis $\mathrm{T} 300 / 934$ composite at $\mathrm{T}=160^{\circ} \mathrm{C}$

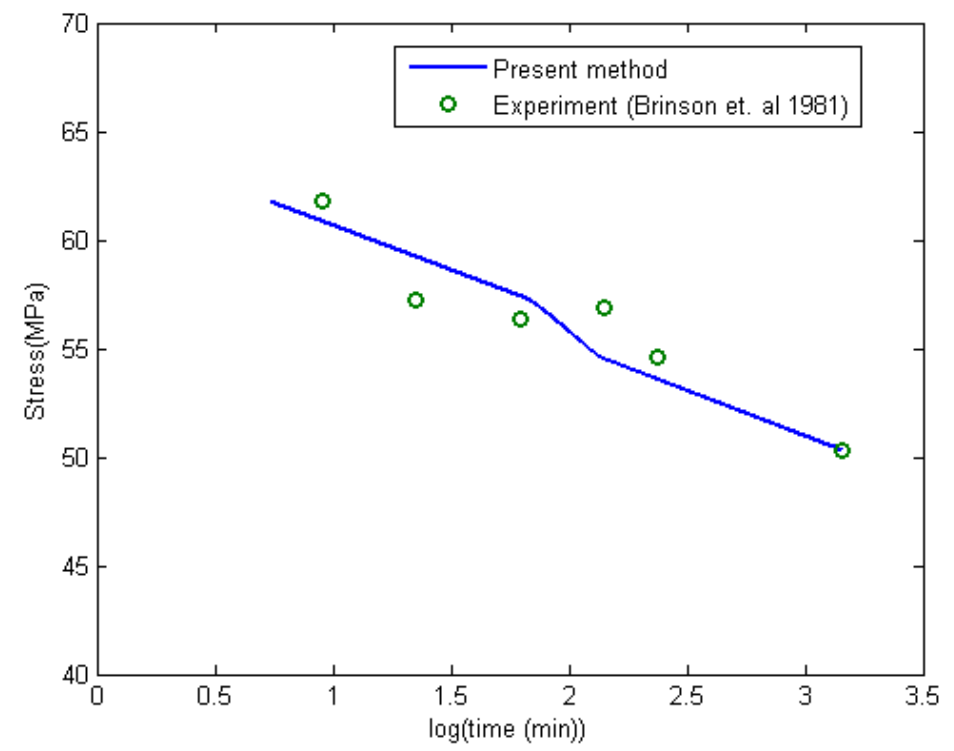

Figure 5-6: Creep failure time for $30^{\circ}$ off-axis $\mathrm{T} 300 / 934$ composite at $\mathrm{T}=160^{\circ} \mathrm{C}$

\subsection{The Effect of Temperature on the Creep Failure}

In this section, the effect of temperature on the creep failure of T300/934 composites is studied through the present approach. The micromechanical model is used to obtain the 
creep failure strength of the matrix material in the transverse direction, i.e. $Y_{\text {mcreep }}$ from the experimental results by Brinson et al (1981) at $193^{\circ} \mathrm{C}$ as follows:

$$
\begin{aligned}
& Y_{\text {mcreep }}=16.64273+1.86415 \exp (-t / 71.42584)+2.58483 \exp (-t / 2103.728) \\
& +2.0662 \exp (-t / 4.83319)
\end{aligned}
$$

However, in the absence of any reliable information on the creep behavior of the composite material in shear loading at this temperature, the same creep failure function is used for both shear and transverse directions i.e. $Y_{\text {mcreep }}=S_{\text {mcreep }}$. Figures 5.7 and 5.8 respectively, show the creep failure responses of the $60^{\circ}$ and the $45^{\circ}$ unidirectional composite at two different temperatures. The disagreement between the present results and the experiments for the higher temperature may be attributed to the foregoing assumption of considering the same creep failure function for both transverse and shear loading. Nevertheless, increasing temperature is seen to have reduced the creep strength of the material drastically for both off axis angles where the behavior of the material is more matrix dominant.

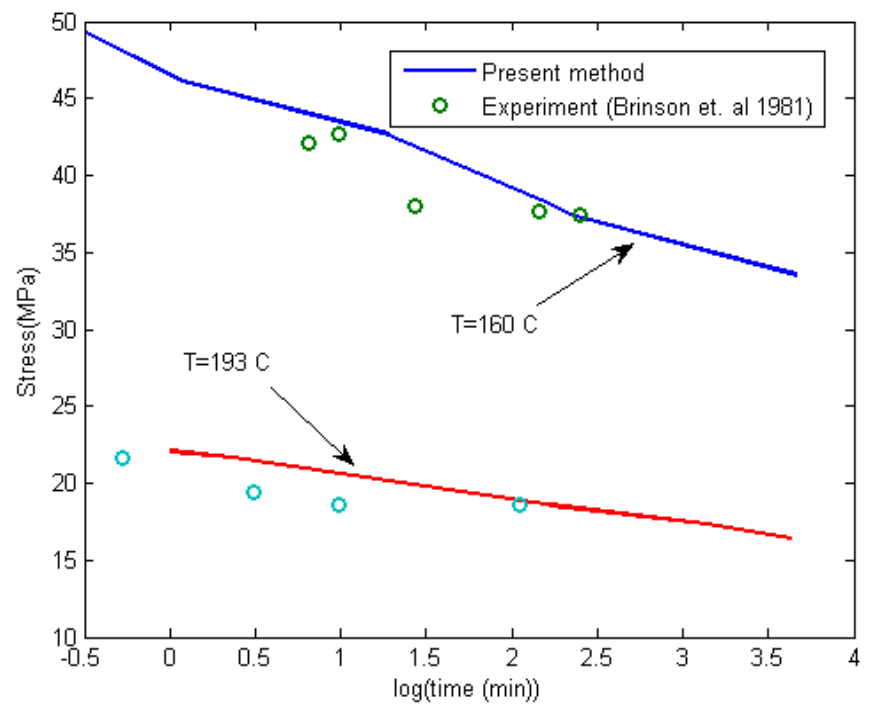

Figure 5-7: Creep failure time for $60^{\circ}$ off-axis T300/934 composite at two different temperatures 


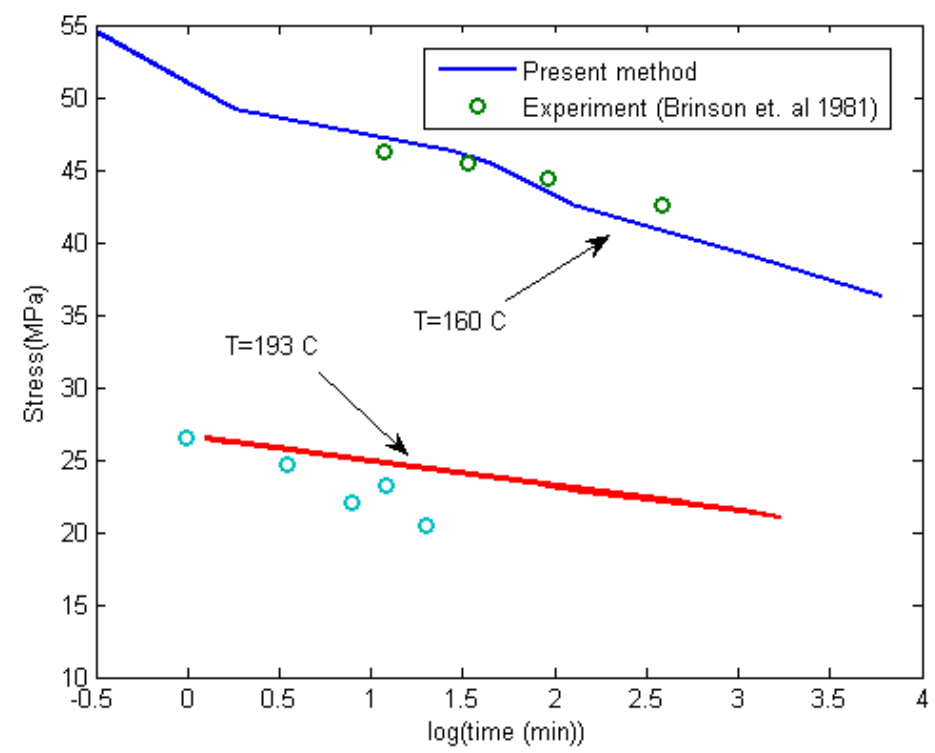

Figure 5-8: Creep failure time for $45^{\circ}$ off-axis T300/934 composite at two different temperatures

\subsection{Conclusion}

A novel approach is introduced to study the time delayed failure of unidirectional polymer matrix composites through a simplified unit cell micromechanical model. The creep failure criterion is expressed in terms of the creep failure strengths of the viscoelastic matrix material in transverse and shear loadings, which are functions of time and temperature. The micromechanical model is also used to calculate these creep failure functions from the knowledge of the creep behavior of the composite material in principal directions. As a result, the creep life of the unidirectional composite material at any off-axis degree can be obtained from testing of the composite in only transverse and shear loading, which eliminates the need for any further experimentation. The results obtained are seen to be in agreement with the experimental data in the literature for different off-axis degrees. The creep strength of the material is seen to have decreased with increasing temperature in for both $60^{\circ}$ and $45^{\circ}$ 
laminates where the polymer matrix plays an important role in the general response of the composite material. 


\section{CHAPTER 6: THE ROLE OF VISCOELASTICITY ON THE FATIGUE OF ANGLE-PLY POLYMER MATRIX COMPOSITES AT HIGH AND ROOM TEMPERATURES}

In this chapter, the micromechanical model is modified to study the role of viscoelasticity on the fatigue behavior of polymer matrix composites. In particular, the study examines the interaction of fatigue and creep in angle ply carbon/epoxy at $25^{\circ} \mathrm{C}$ and $114^{\circ} \mathrm{C}$. The matrix phase is modeled as a vicoelastic material using Schapery's single integral constitutive equation. Taking viscoelsticity into account allows the study of creep strain evolution during the fatigue loading. The fatigue failure criterion is expressed in terms of the fatigue failure functions of the constituent materials. The main goal of this chapter is to study the fatigue failure of angle-ply polymer matrix composites including the hereditary effect of the matrix material which can thus study effects of static-fatigue (creep) and dynamic degradation of is strength due to the applied cyclic loading, separately both at room and high temperatures.

\subsection{Interaction of Creep and Fatigue}

In fatigue loading of polymer matrix composites, the mean stress can be regarded as a static load under which the material creeps. Based on this assumption the mean strain developed in the material under fatigue loading may be written as

$\Delta \varepsilon=\Delta \varepsilon_{\text {cyclic }}+\Delta \varepsilon_{\text {creep }}$

where $\Delta \varepsilon_{\text {creep }}$ is the portion of the strain accumulated in the material due to the viscoelasticity of the polymer matrix. $\Delta \varepsilon_{c y c l i c}$ denotes the portion of the strain development due to cyclic or fatigue damage. Both these portions are functions of temperature, time and the mean stress. A comprehensive fatigue model should therefore be able to account for both of these portions 
of strain development. The fatigue failure criterion used in this work expresses the failure of matrix and fiber constituents in terms of the average stresses of the fiber and matrix subcells as follows:

$$
\left|\frac{\sigma^{(I)}{ }_{11}}{X_{f}}\right|=1
$$

$\left[\frac{\sigma^{(k)}{ }_{22}}{X_{m}}\right]^{2}+\left[\frac{\sigma^{(k)}{ }_{12}}{S_{m}}\right]^{2}=1$

In the above-mentioned equation, $\mathrm{k}=\mathrm{II}, \mathrm{III}, \mathrm{IV}$ refer to various matrix subcells. The stresses in the fiber and matrix subcells are obtained using the Simplified Unit Cell Micromechanical model. This enables the direct consideration of the effect of viscoelasticity of the matrix material in calculating the local stresses $\sigma_{22}^{(k)}$ and $\sigma_{12}^{(k)}$. Unlike the static loading $X_{m}, X_{f}$ and $S_{m}$ are the fatigue failure functions of the constituent materials which depend on stress amplitude R, frequency, temperature and the number of cycles. The failure is assumed to have occurred once one of the subcells fails the forgoing criterion. The present methodology is capable of calculating the contribution to the total strain development, made by fatigue damage and viscoelasticity, i.e. both terms on the right-hand side of Equation 6.1, separately. In order to model stiffness degradation due to cycling, the model developed by Ramakrishnan and Jayaraman (1993) is utilized as follows assuming that the bulk of stiffness degradation and subsequently the damage happen in the matrix material:

$$
\frac{E}{E_{c}}=1-\left[\frac{E_{m} V_{m}}{E_{c}}\left((1-f) \frac{\ln (N+1)}{\ln \left(N_{f}\right)}+f \frac{N}{N_{f}}\right)\right]
$$


In Equation 6.3, $E_{c}$ denotes the composite stiffness. The terms $V_{m}$ and $E_{m}$ correspond to matrix volume fraction and modulus, respectively and $N_{f}$ indicates the number of cycles to failure of the composite material. $f$ is a factor accounting for fiber-matrix interface strength which varies between 0 (poor fiber-matrix interface) and 1 (perfectly bonded fiber matrix interface). The assumption that damage is mainly due to stiffness degradation in the matrix material holds true for higher fiber angles when the behavior of the material is matrix dominated. The problem however, is that Equation 6.3 needs the fatigue life of the material, $N_{f}$, as an input whereas finding $N_{f}$ is one of the objectives in the present approach. In order to alleviate this problem, a convergence algorithm as shown in figure 6.1 is incorporated into the formulation. An educated guess for $N_{f}$ which could ensure convergence after only a few iterations would be obtained based on the assumption that the stiffness degradation of the matrix material is mainly due to loading in the transverse direction in other words, mode I of fracture. For an angle ply lamina subjected to a general state of loading in the global coordinate system $\sigma_{x}, \sigma_{y}$ and $\tau_{x y}$, the stress components in the lamina coordinate system are found through the stress transformation matrix [T1] (Agarwal et al., 2006):

$$
\left\{\begin{array}{l}
\sigma_{L} \\
\sigma_{T} \\
\tau_{L T}
\end{array}\right\}=\left[T_{1}\right]\left\{\begin{array}{l}
\sigma_{x} \\
\sigma_{y} \\
\tau_{x y}
\end{array}\right\}
$$

Once $\sigma_{T}$ is obtained from Equation 16, its corresponding $N_{f}$ i.e. the number of cycles to failure can be calculated from the S-N curve of the composite material in transverse direction, which serves as the first guess to be input into the convergence algorithm of figure 6.1 . 


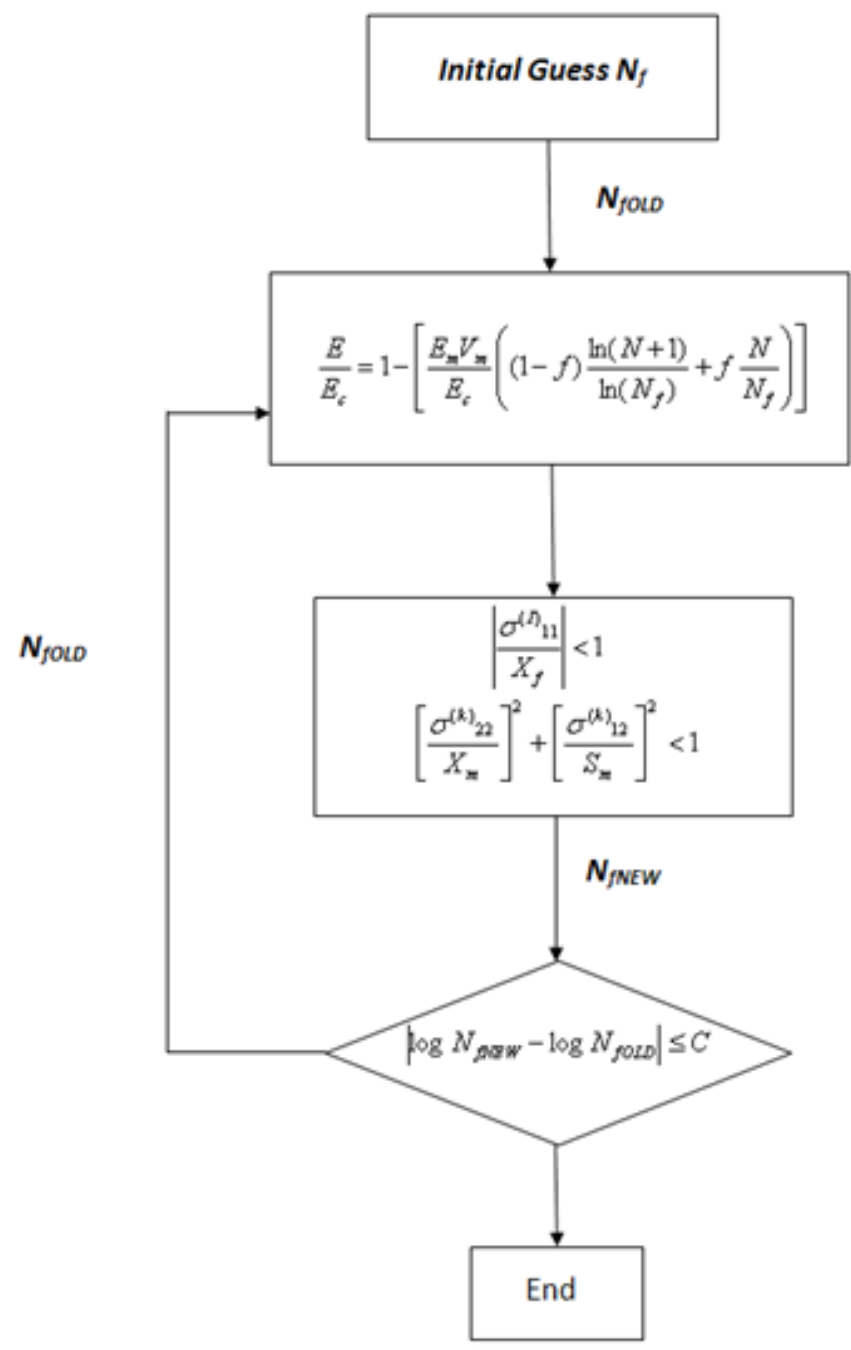

Figure 6-1: Convergence algorithm to find fatigue life of the composite

\subsection{Creep Behavior of Unidirectional E-Glass/Epoxy}

To demonstrate the capability of the present micromechanical approach to account for the viscoelasticity of polymer matrix composites, the SUCM model is first validated through comparison with creep test results for E-glass/epoxy off -axis composites reported by Lou and Schapery (1971). The material properties of composite constituents and the Prony series coefficients for the epoxy matrix are tabulated in Tables 6.1 and 6.2, respectively. 
Table 6-1: Glass and epoxy elastic material properties, $V_{f}=0.476$ (Haj-Ali and Muliana, 2004a)

\begin{tabular}{|l|c|c|}
\hline \multicolumn{2}{|c|}{$E(\mathrm{GPa})$} & $\mathrm{V}$ \\
\hline Glass fiber & 72.4 & 0.22 \\
\hline Epoxy matrix & 4.3 & 0.31 \\
\hline
\end{tabular}

Table 6-2: Prony series coefficients for the matrix material (Haj-Ali and Muliana, 2004a)

\begin{tabular}{|c|c|c|}
\hline$n$ & $\lambda_{n}$ & $D_{n}\left(\mathrm{MPa}^{-1}\right)$ \\
\hline 1 & 1 & $2.1758 \mathrm{e}-6$ \\
\hline 2 & $1 \mathrm{e}-1$ & $4.8738 \mathrm{e}-6$ \\
\hline 3 & $1 \mathrm{e}-2$ & $5.0769 \mathrm{e}-6$ \\
\hline 4 & $1 \mathrm{e}-3$ & $6.6435 \mathrm{e}-6$ \\
\hline 5 & $1 \mathrm{e}-4$ & $1.8277 \mathrm{e}-6$ \\
\hline 6 & $1 \mathrm{e}-5$ & $2.9011 \mathrm{e}-6$ \\
\hline
\end{tabular}

Figures 6.2 and 6.3 show the creep responses for the $60^{\circ}$ and the $90^{\circ}$ off-axis specimens, respectively. The results are in good agreement with the experimental data which thus validate the present viscoelastic formulation. In both cases, the acceleration of the creep strain and softening of the initial and time-dependent portions of the composite response increases with increasing stress. In particular, as can be seen in figure 6.2 , the material exhibits nonlinear behavior for the highest stress value i.e. $g_{i}$ 's in Schapery's are greater than unity. 


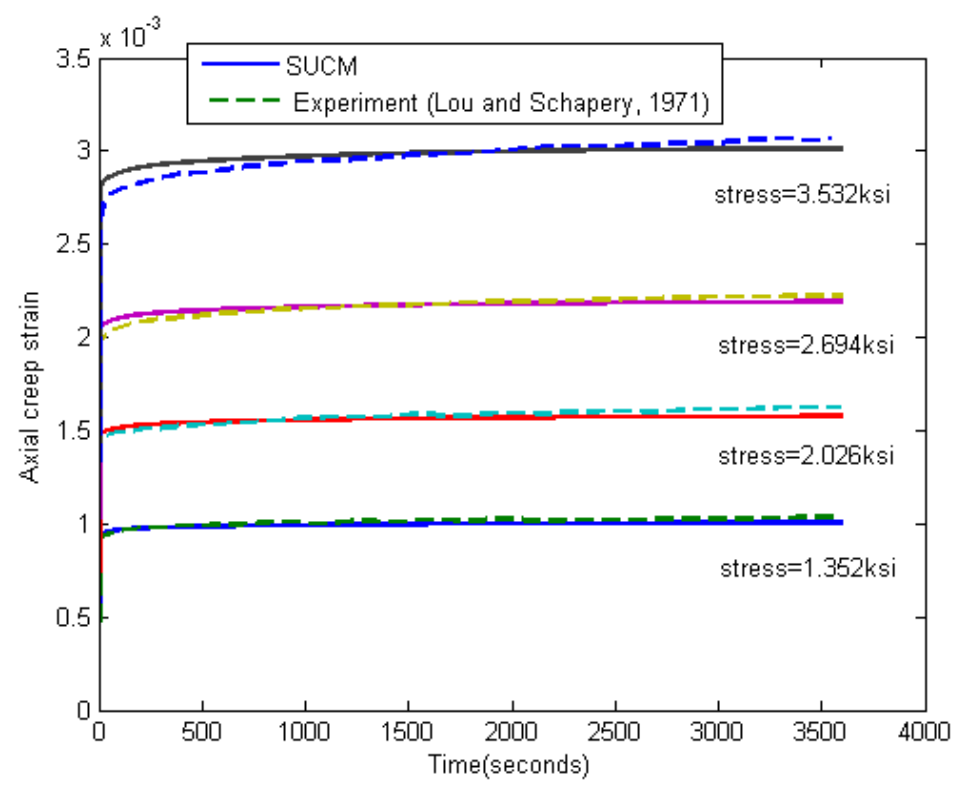

Figure 6-2: Axial creep strain for $60^{\circ}$ off-axis Glass/Epoxy composite

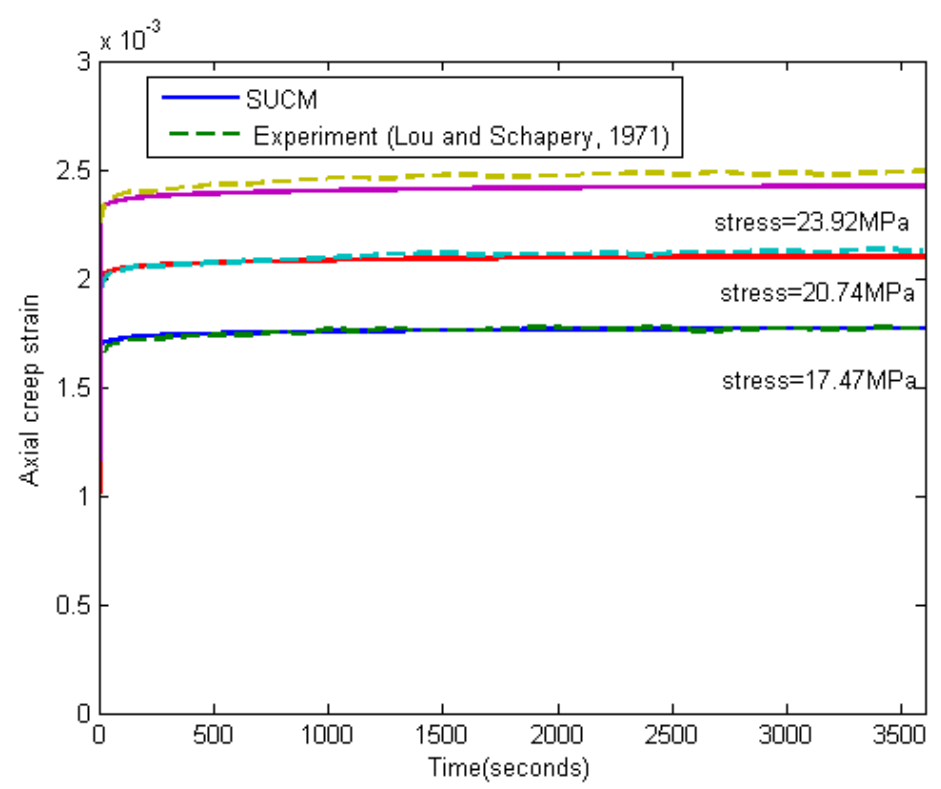

Figure 6-3: Axial creep strain for $90^{\circ}$ off-axis Glass/Epoxy composite

\subsection{Fatigue behavior of unidirectional E-Glass/Epoxy}

Unidirectional E-glass/epoxy off-axis composite specimens with a fiber volume fraction of $60 \%$ were tested for fatigue failure up to $10^{6}$ cycles at the frequency of $19 \mathrm{cps}$ by Hashin and Rotem 
(1973). The stress ratio of all the tests was 0.1. In this section the SUCM model is utilized to predict the fatigue failure of unidirectional E-glass/epoxy composites in off-axis loading including the effect viscoelasticity. Figures 6.4 and 6.5 depict the S-N diagrams of the composite material in principal directions. As previously mentioned, these data are then used to generate fatigue strengths of the composite constituents as a function of the number of cycles for the specified frequency and stress ratio using the micromechanical model, which are input into the fatigue failure criterion of Equation 6.2.

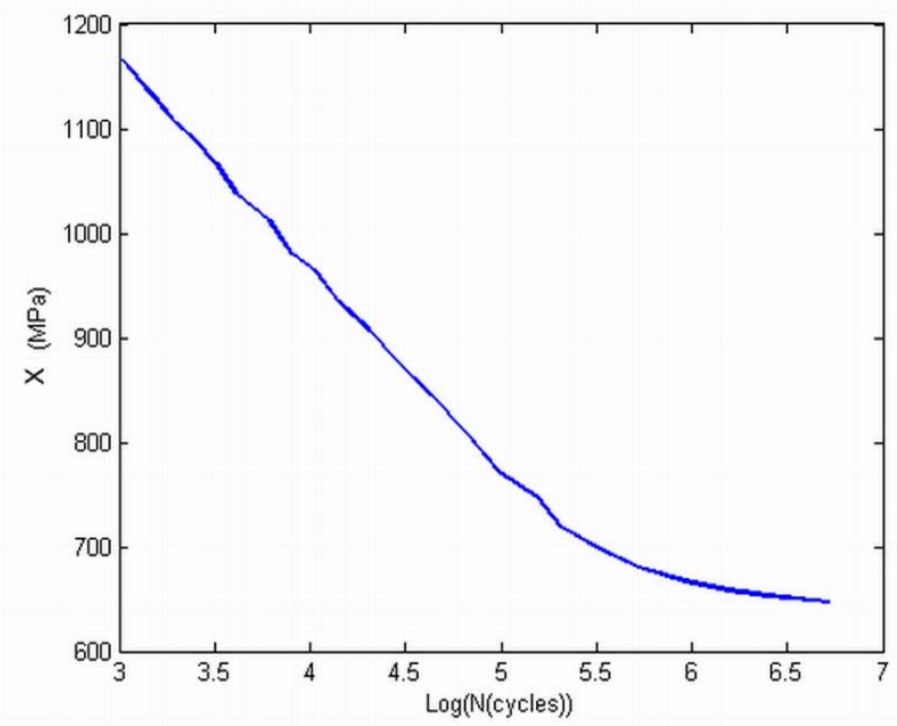

Figure 6-4: Longitudinal fatigue failure diagram of E-Glass/Epoxy composite (Hashin and Rotem, 1973) 


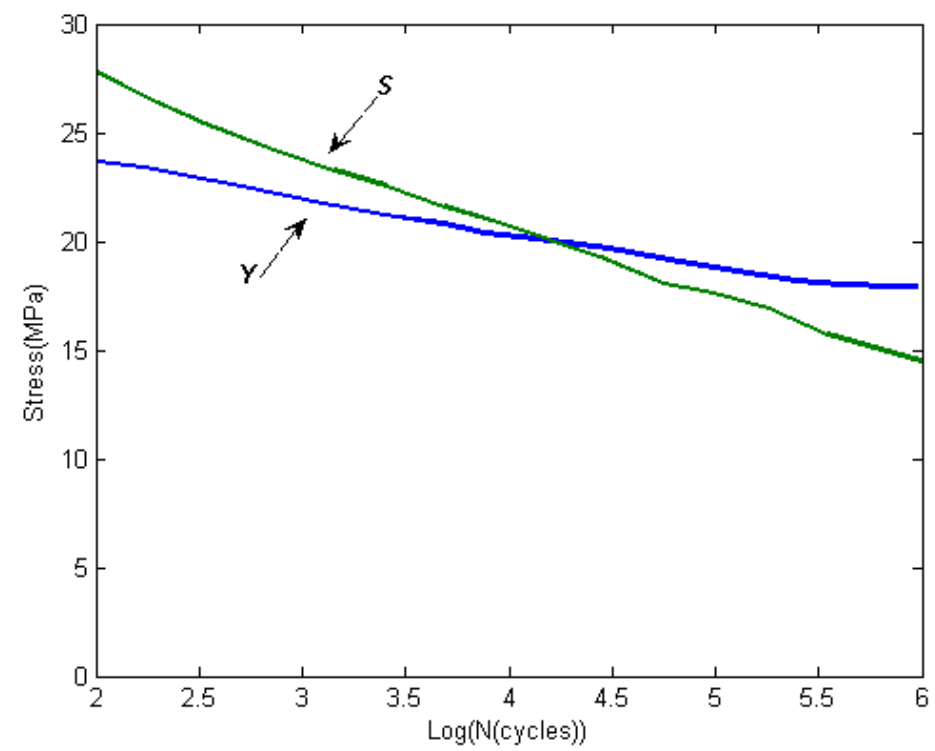

Figure 6-5: Transverse Fatigue and shear fatigue failure diagram of E-Glass/Epoxy composite (Hashin and Rotem, 1973)

Figures 6.6-6.8 show a comparison between the results of the present study with the literature. The results are seen to be in good agreement with the experimental data. As expected, the results show the degradation of strength with the number of cycles. In concurrence with the experimental observations reported in the reference, two modes of failure were found to be dominant in determining the fatigue life of the composite specimens: fiber fracture and matrix cracking. The micromechanical approach as opposed to the macromechanics can distinguish between these two failure modes. 


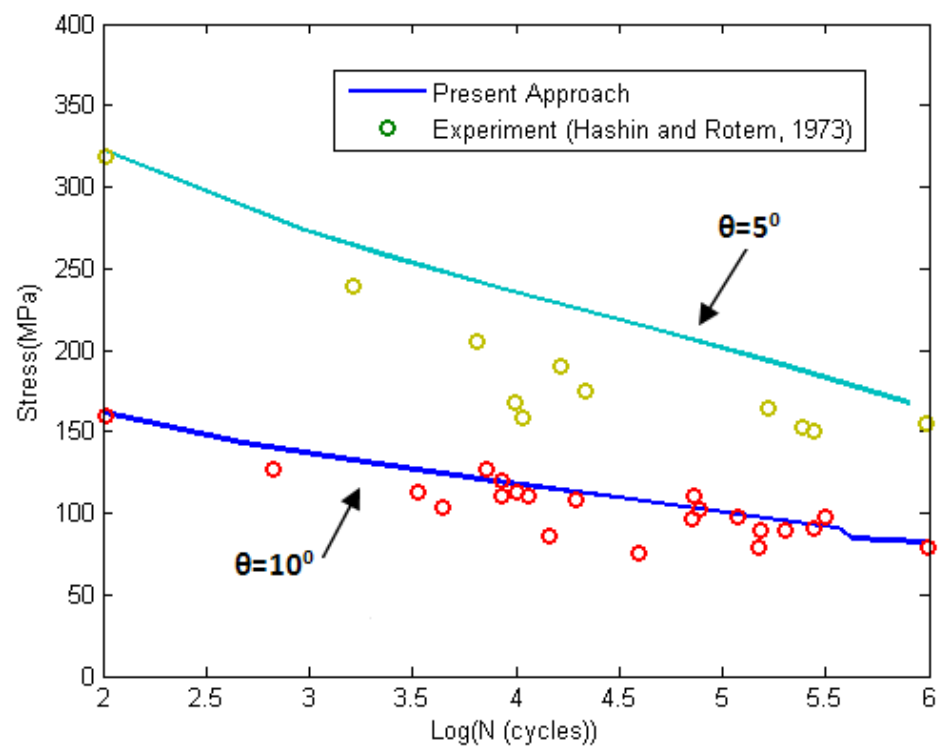

Figure 6-6: Fatigue failure diagram of off-axis Glass/Epoxy composite, $\theta=5^{\circ}$ and $\theta=10^{\circ}$

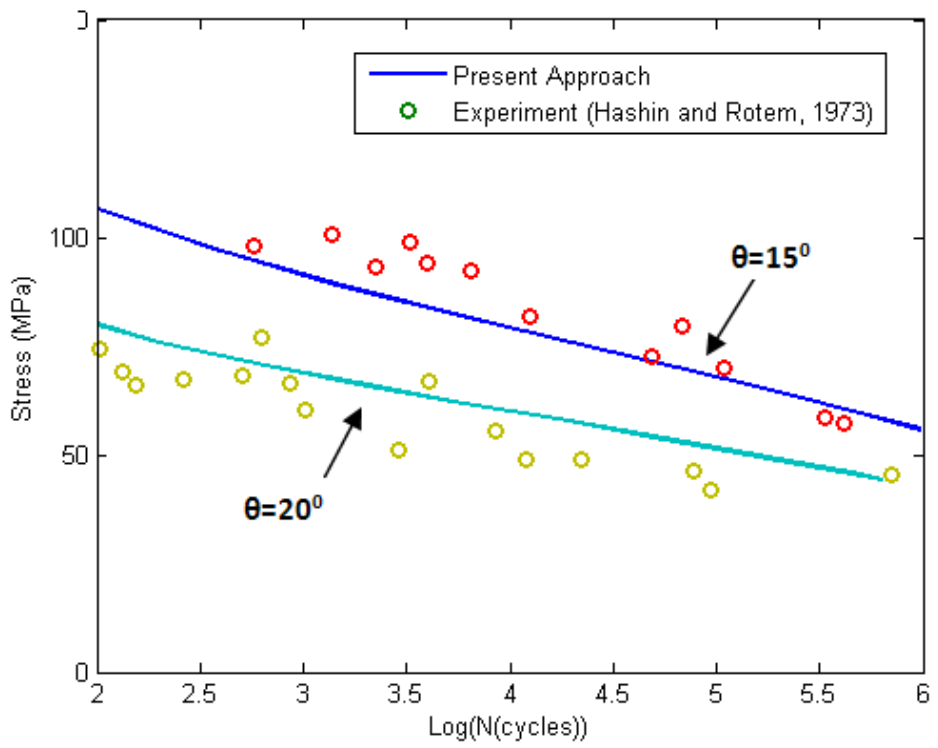

Figure 6-7: Fatigue failure diagram of off-axis Glass $/$ Epoxy composite, $\theta=15^{\circ}$ and $\theta=20^{\circ}$ 


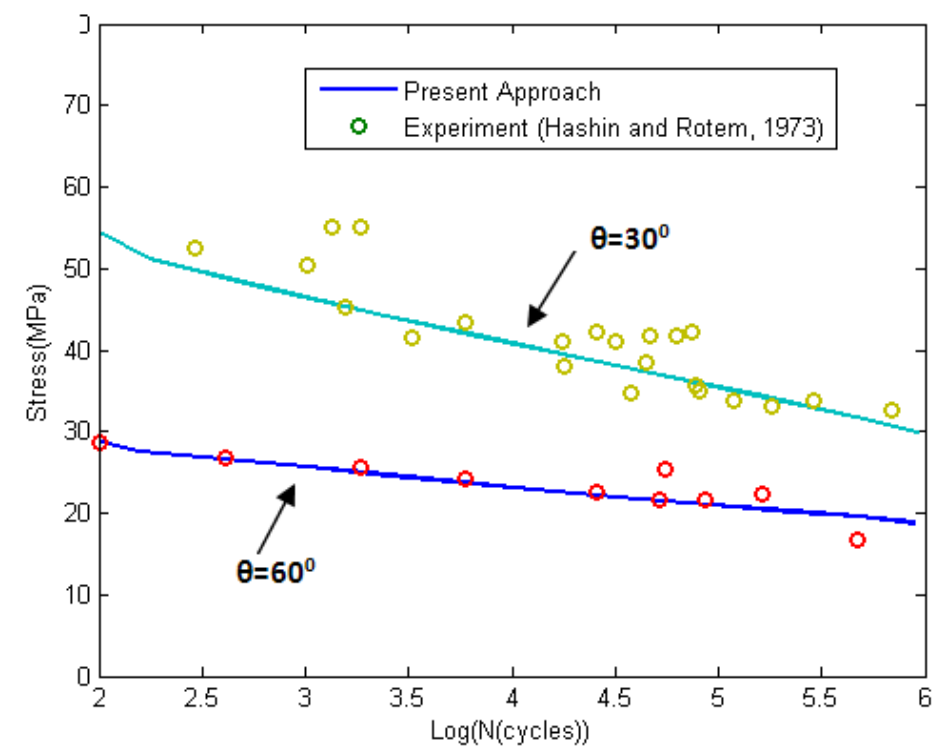

Figure 6-8: Fatigue failure diagram of off-axis Glass $/$ Epoxy composite, $\theta=30^{\circ}$ and $\theta=60^{\circ}$

Including viscoelasticity in the constitutive relation of the matrix material enables the present model to calculate the strain $\Delta \varepsilon_{\text {creep }}$ which is the result of creep in the material under the fatigue mean stress. This is in fact the difference between the fatigue mean strains with and without including the viscoelasticity in the formulation. Since the mean strain is on the rise during fatigue, $\max \left(\Delta \varepsilon_{\text {creep }}\right)$ takes place at the end of the fatigue life of the material. This is schematically shown in figure 6.9. Figure 6.10 shows the maximum viscoelastic strain developed corresponding to different stress levels for the $60^{\circ}$ composite off-axis laminate. It can be seen that increasing the mean stress reduces the viscoelastic strain accumulation in the material. The fact is that although viscoelasticity is more pronounced at higher stress levels, its contribution to strain development during fatigue is compromised by the shorter life of the specimen when compared to lower stresses. This accumulation of viscoelastic strain during fatigue loading accelerates the failure of the material. This is apparent in figure 6.11 which shows the S-N curve for the $60^{\circ}$ off-axis specimen with and without viscoelasticity. In fact, as 
the off-axis angle increases, the behavior of the material becomes more matrix dominated and the present method taking the viscoelasticity of the matrix material into account, yields better results. However, the importance of viscoelasticity in predicting the fatigue life of polymer matrix composite materials is expected to be more pronounced at elevated temperatures.

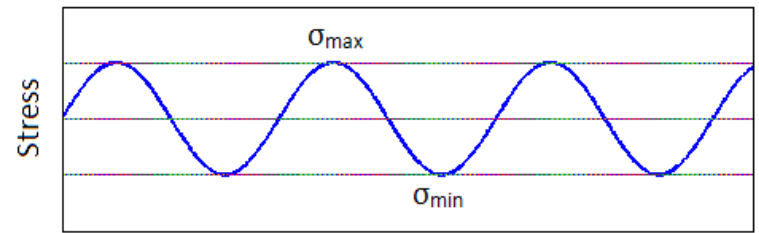

Number of Cycles

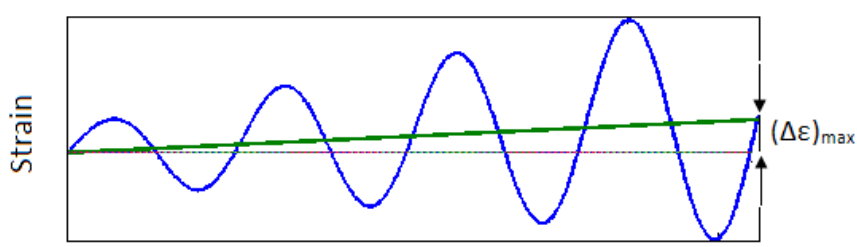

Number of Cycles

Figure 6-9: Schematic diagram of stress-controlled fatigue

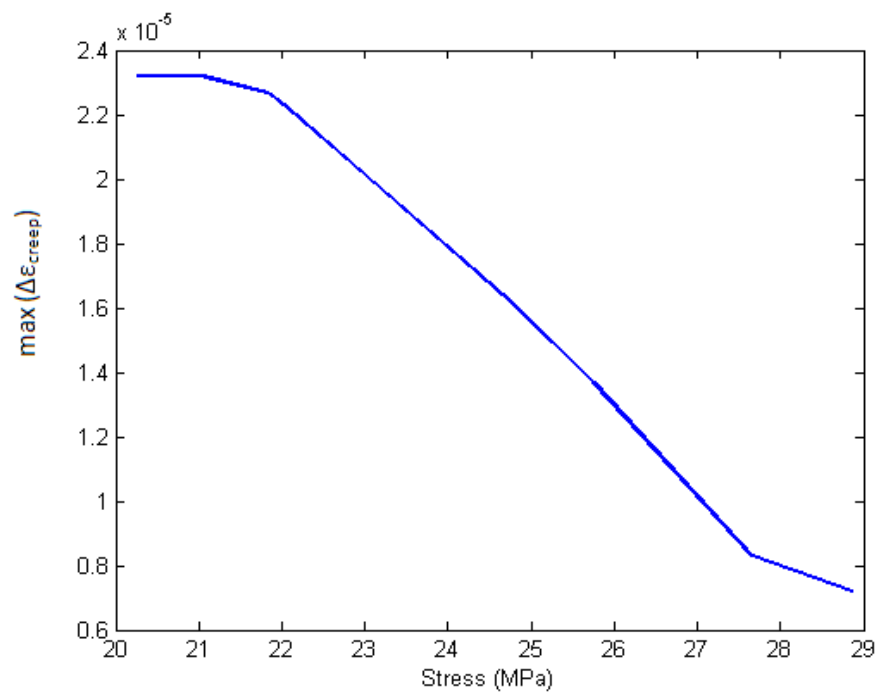

Figure 6-10: Maximum accumulated viscoelastic strain for the $60^{\circ}$ composite off-axis laminate 


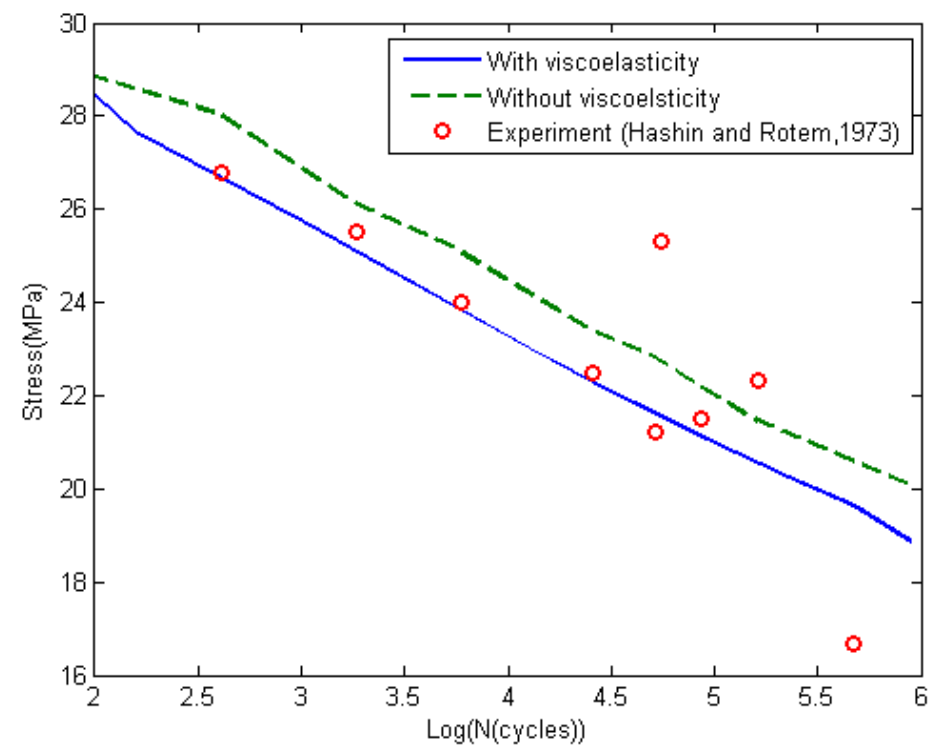

Figure 6-11: Fatigue failure diagram of off-axis Glass/Epoxy composite, $\theta=60^{\circ}$ with and without considering the effect of viscoelasticity

\subsection{Creep Response of T300/5208}

The material selected for this study is a graphite/epoxy composite made up of T300 fibers and 5208 epoxy with $65 \%$ volume fraction which has extensive applications in the aerospace industry. The time temperature shift factor of 5208 epoxy resin has been extracted from thermo-mechanical and electrical conductivity measurements by Kibler and Carter (1978). The elastic properties of the composite materials and the Prony series coefficients calibrated for the linear viscoleastic response of the matrix material are tabulated in Tables 6.3 and 6.4, respectively. The nonlinear coefficients $g_{0}, g_{1}$ and $g_{2}$ were taken from the reference (Haj-Ali and Muliana, 2004a).

Table 6-3: Graphite and epoxy elastic material properties (Haj-Ali and Muliana, 2004a)

\begin{tabular}{|l|c|c|c|c|c|}
\hline & $E_{11}(\mathrm{GPa})$ & $E_{22}(\mathrm{GPa})$ & $G_{12}(\mathrm{GPa})$ & $v_{12}$ & $v_{23}$ \\
\hline Graphite fiber & 200 & 13 & 44 & 0.39 & 0.40 \\
\hline Epoxy matrix & 4.6 & & & 0.35 & \\
\hline
\end{tabular}


Table 6-4: Prony Series coefficient for 5208 Epoxy matrix (Haj-Ali and Muliana, 2004a)

\begin{tabular}{|c|c|c|}
\hline$n$ & $\lambda_{n}$ & $D_{n}\left(\mathrm{MPa}^{-1}\right)$ \\
\hline 1 & 1 & $8.50 \mathrm{e}-6$ \\
\hline 2 & $1 \mathrm{e}-1$ & $8.36 \mathrm{e}-6$ \\
\hline 3 & $1 \mathrm{e}-2$ & $5.50 \mathrm{e}-6$ \\
\hline 4 & $1 \mathrm{e}-3$ & $33.80 \mathrm{e}-6$ \\
\hline
\end{tabular}

Tuttle and Brinson (1986) subjected $10^{\circ}$ and $90^{\circ}$ off-axis unidirectional T300/5208 specimens to creep tests for $480 \mathrm{~min}$ at different stress levels. These tests were performed at room temperature. Figures 6.12 and 6.13 show the transverse and shear creep strains of the unidirectional composite. Good-to-reasonable agreement with the experimental results shows that the Simplified Unit Cell Micromechanical (SUCM) model is capable of predicting the viscoelastic response of the T300/5208 composite. Figure 6.14 shows the transverse creep response of $\mathrm{T} 300 / 5208$ at $25^{\circ} \mathrm{C}$ and $114^{\circ} \mathrm{C}$. The results for the high temperature were obtained by changing the shift factor based on its temperature dependence determined by Kibler and Carter (1978). It can be seen that increasing temperature gives rise to the softening of the material resulting in higher strains in both the initial and time-dependent portions of the composite response. This increase is of course more prominent for higher stress levels when the matrix material enters the nonlinear viscoelastic range. 


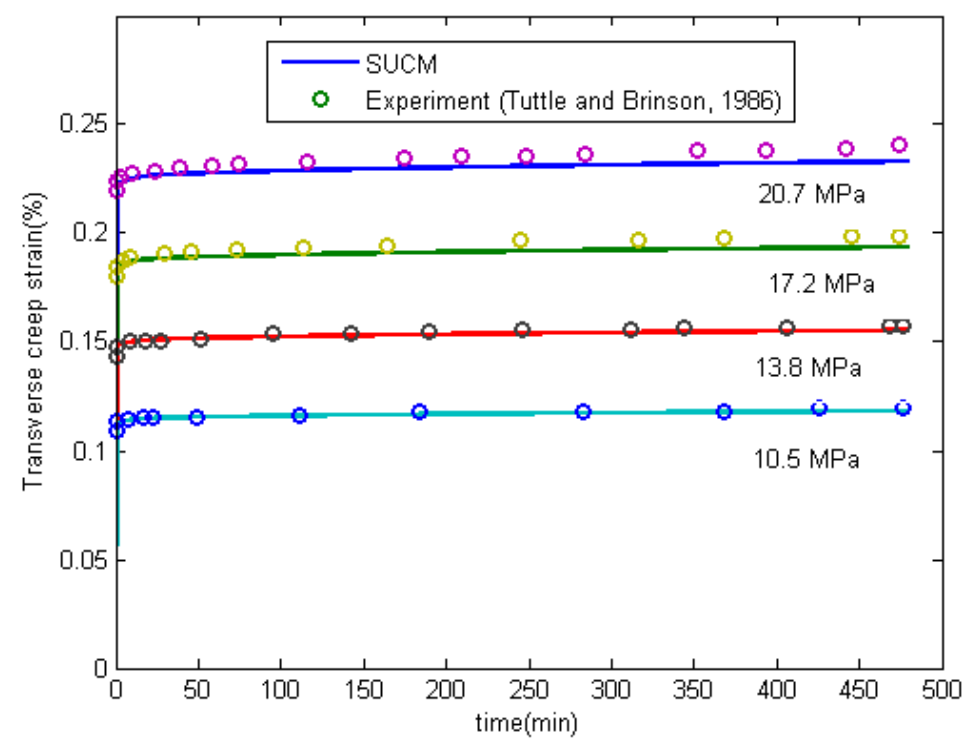

Figure 6-12: Transverse creep strain for T300/5208 unidirectional composite at different stress levels

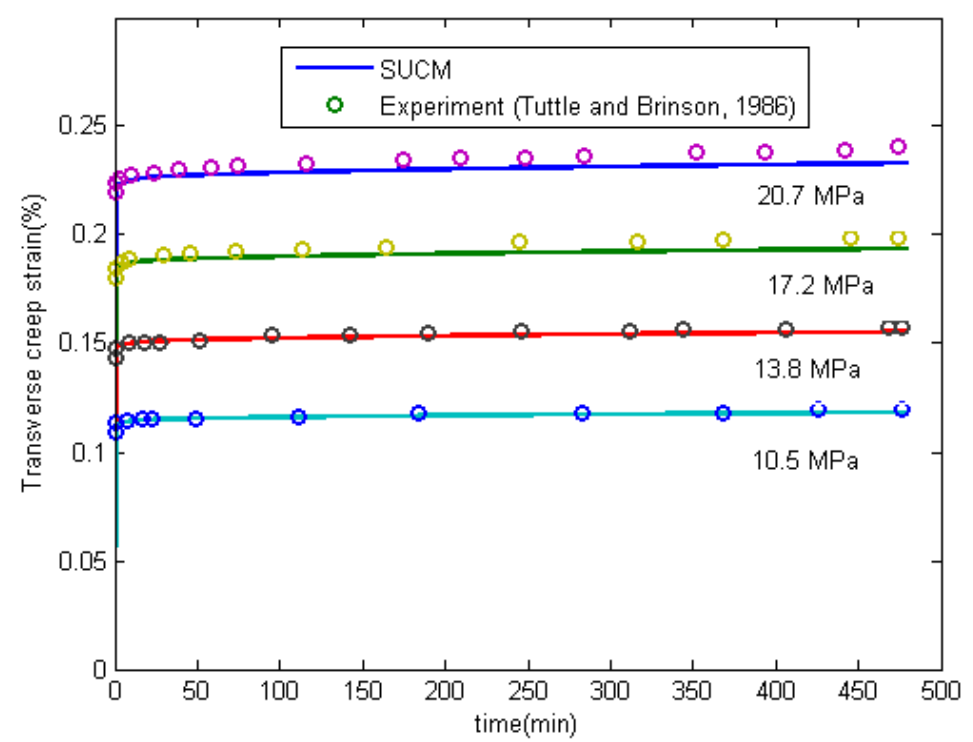

Figure 6-13: Shear creep strain for $10^{\circ}$ off-axisT300/5208 unidirectional composite at different stress levels 


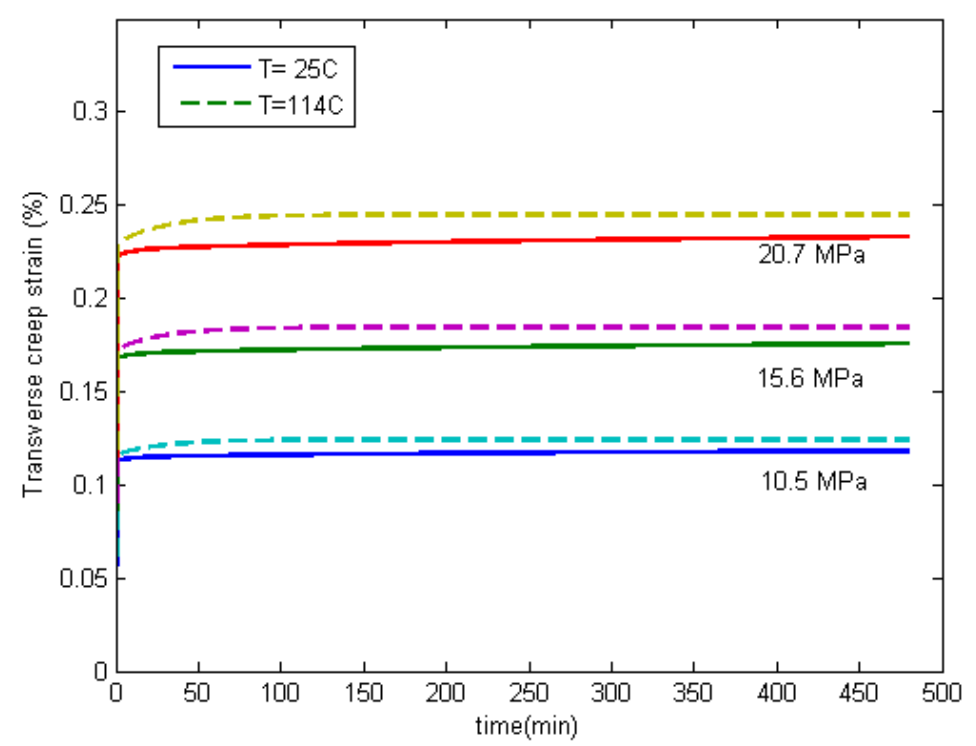

Figure 6-14: Transverse creep response of T300/5208 at $25^{\circ} \mathrm{C}$ and $114^{\circ} \mathrm{C}$

\subsection{Fatigue Behavior of Angle-Ply T300/5208}

Tension-tension fatigue tests on unidirectional, angle-ply and symmetrically balanced laminates at different temperatures were carried out by Rotem and Nelson at a frequency of $10 \mathrm{cps}$ (Rotem and Nelson, 1978). The stress ratio of all tests was 0.1. In this section the SUCM model is utilized to predict the fatigue failure of $[ \pm 75]_{2 s} T 300 / 5208$ composites including the effect of viscoelasticity and damage accumulation of the matrix material. Figure 6.15 depicts the S-N diagrams of the composite material in principal directions at $25^{\circ} \mathrm{C}$ and $114^{\circ} \mathrm{C}$. As previously mentioned, these data are then used to generate fatigue strengths of the composite constituents as a function of the number of cycles for the specified frequency and stress ratio using the micromechanical model, and are then input into the fatigue failure criterion of Equation 6.2. It can be seen that while the longitudinal and shear strengths of the composite material remain unchanged with temperature, the transverse strength decreases with increasing temperature making the material more susceptible to failure. 

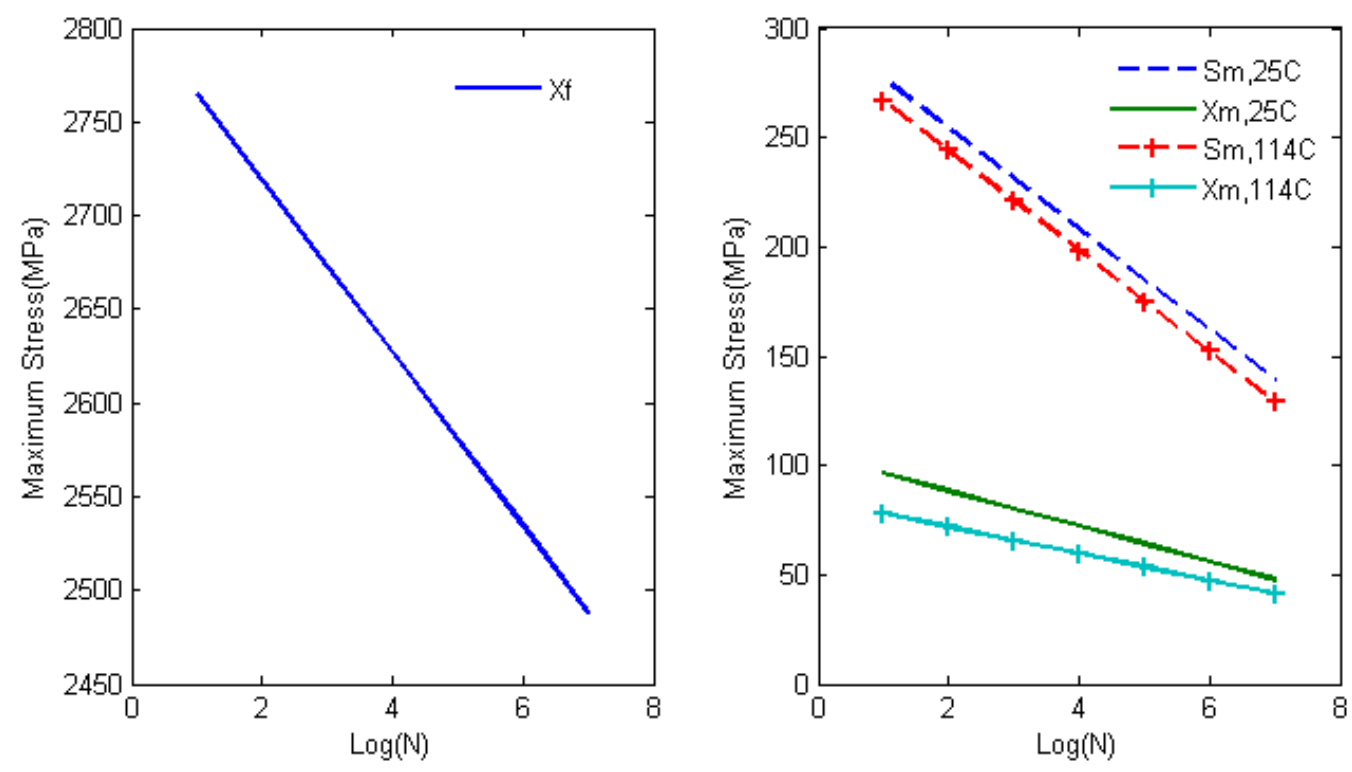

Figure 6-15: S-N diagrams of the composite material in principal directions at $25^{\circ} \mathrm{C}$ and $114^{\circ} \mathrm{C}$

Figures 6.16 and 6.17 show the S-N curves of $[ \pm 75]_{2 s}$ angle-ply laminate compared to the experimental data reported in the literature. The results are seen to be in good agreement with the experimental data. As expected, the results show the degradation of strength with the number of cycles. In concurrence with the experimental observations reported in the reference, two modes of failure were found to be dominant in determining the fatigue life of the composite specimens: fiber fracture and matrix cracking. The micromechanical approach as opposed to the macromechanics can distinguish between these two failure modes. 


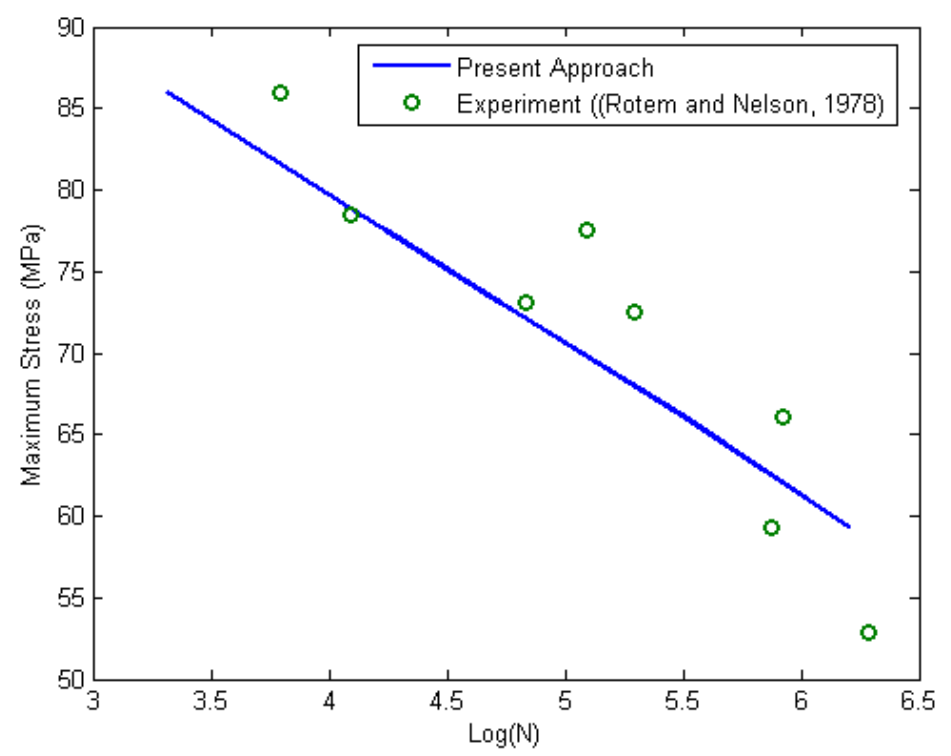

Figure 6-16: S-N curves of $[ \pm 75]_{25}$ angle-ply laminate at $25^{\circ} \mathrm{C}$

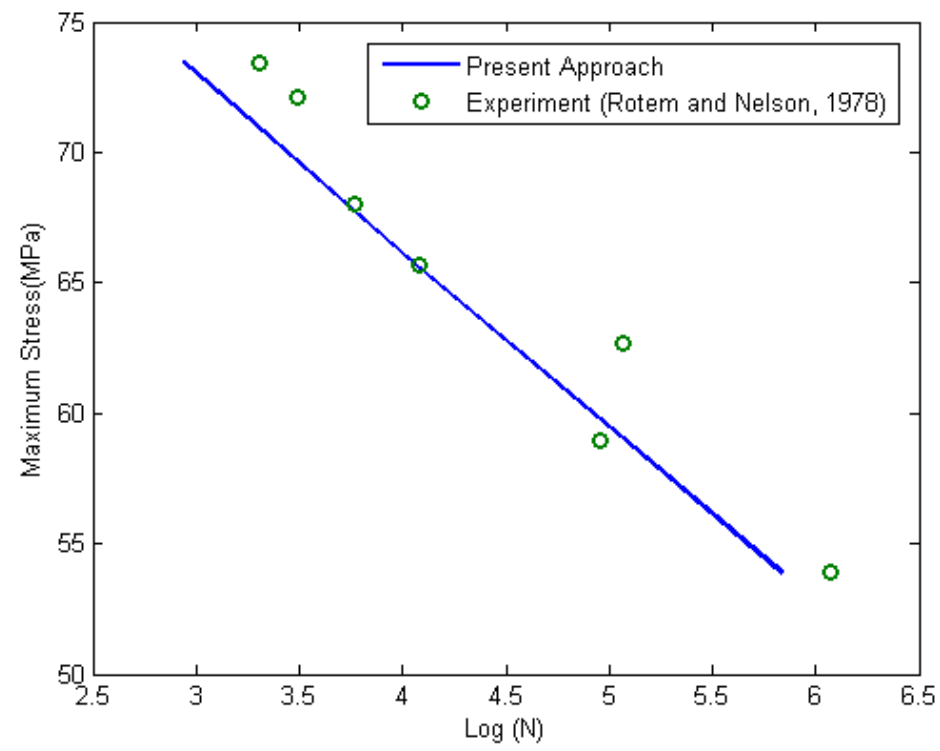

Figure 6-17: S-N curves of $[ \pm 75]_{2 s}$ angle-ply laminate at $114^{\circ} \mathrm{C}$

\subsection{Creep- Fatigue Interaction}

Now that the fatigue life of the composite material has been estimated, the present methodology can be utilized to calculate each term on the right hand side of Equation 6.1, i.e. 
$\Delta \varepsilon_{\text {cyclic }}$ and $\Delta \varepsilon_{\text {creep. }}$. Eliminating viscoelasticity from the formulation will cause the strain development to be the result of fatigue damage. Similarly, if the formulation excludes strength degradation due to fatigue, the resulting strain development will be due to creep or viscoelasticity of the matrix material. Figure 6.18 shows the transverse mean strain development due to fatigue and creep, separately. It can be seen that the share of viscoelasticity is dominant throughout most of the life of the specimen. However, towards the end of the life, it is the stiffness degradation due to fatigue damage that prevails in the evolution of damage.

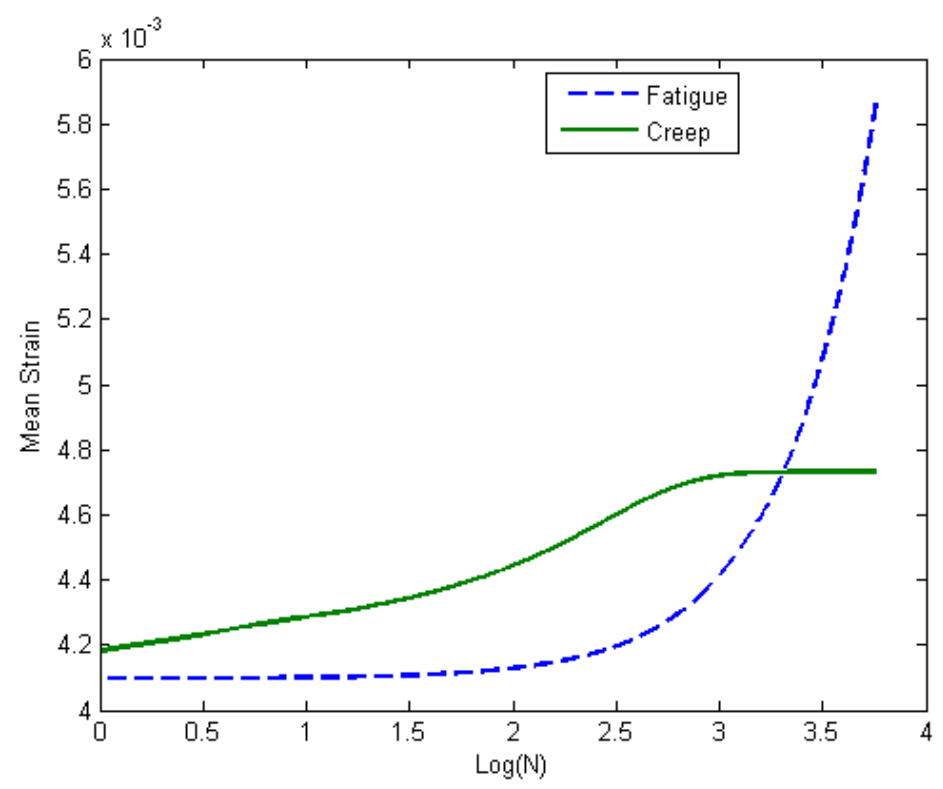

Figure 6-18: Transverse mean strain development due to fatigue and creep

Figure 6.19 depicts the contribution made by the effect of viscoelasticity to the total strain evolution during the fatigue life of the specimen under the same loading conditions at two different temperatures. The distance between the curves can be regarded as a metric for measuring the contribution made by viscoelastity to the overall damage evolution in the 
material. A closer observation of the figure shows that for the lower temperature i.e. $25^{\circ} \mathrm{C}$, the development of fatigue mean strain is mainly dominated by creep. Meanwhile the damage evolution for the higher temperature is governed more by the first term of Equation 6.1. This is an interesting finding in the sense that although viscoelasticity is more pronounced at higher temperature, its contribution to strain development during fatigue is compromised by the shorter life of the specimen when compared to lower temperatures. The effect of mean stress on the contribution of viscoelasticity to the total strain development is shown in figure 6.20. It is clear that for the higher mean stress, although the creep strain rises, the total mean strain development is dominated by stiffness degradation due to cycling i.e. the first term of the right-hand side of Equation 13. This is similar to the effect of high temperature shown in figure 10. In other words, the higher mean stress expedites the failure of the material mainly through cycling as compared to viscoelasticity.

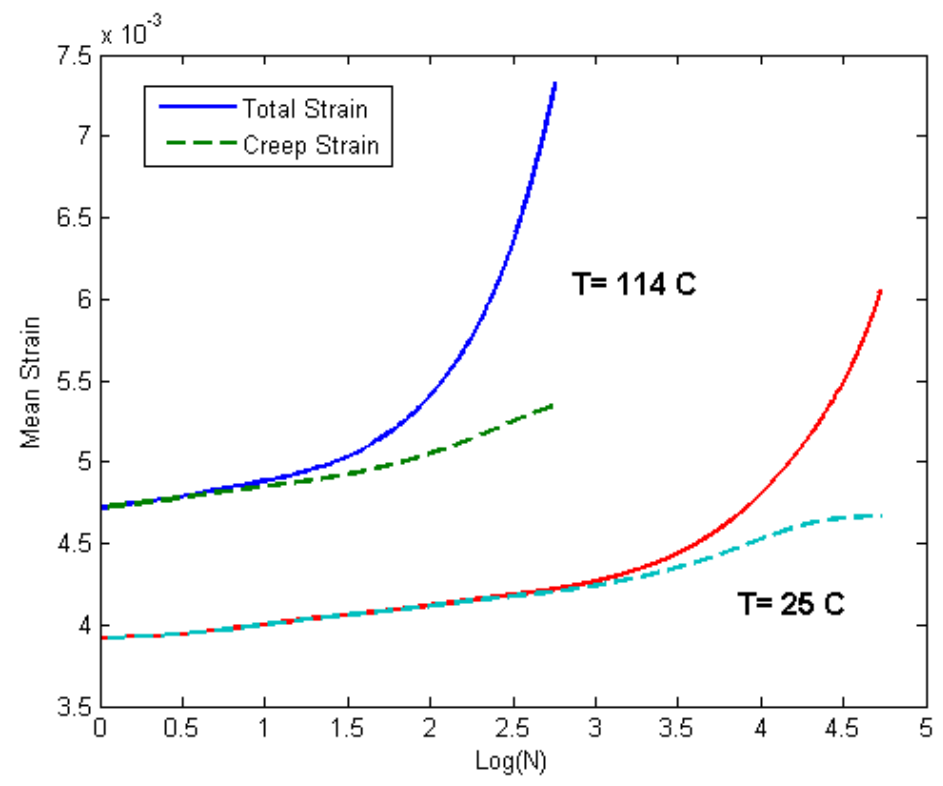

Figure 6-19: Contribution made by viscoelasticity to the total strain evolution at two different temperatures 


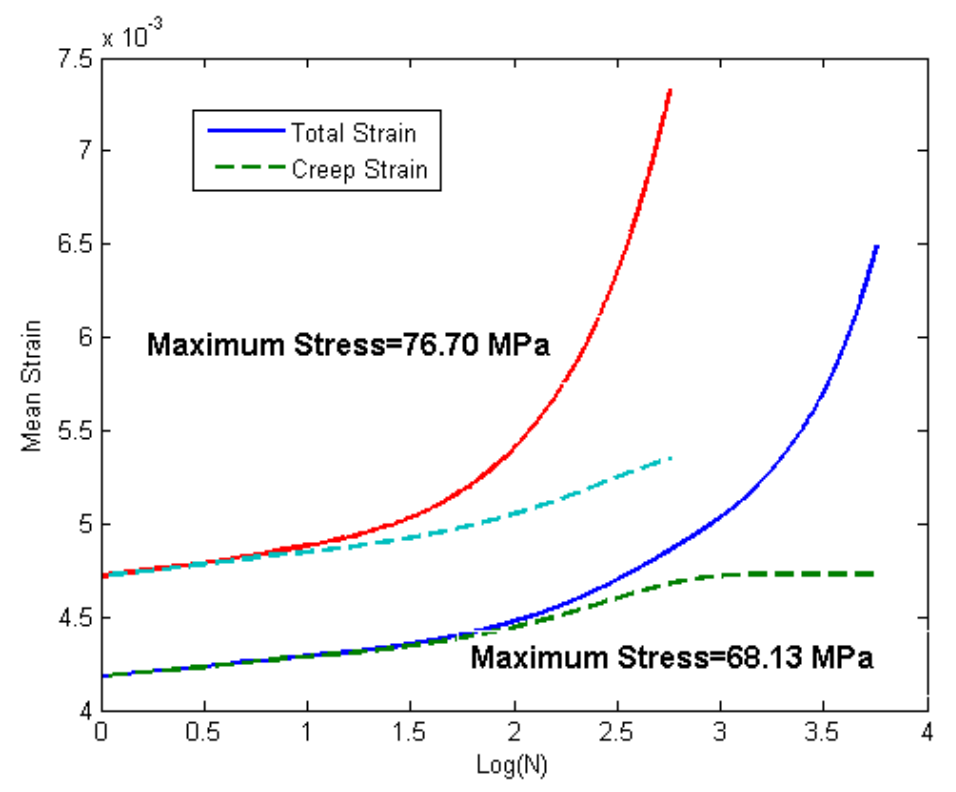

Figure 6-20: The effect of mean stress on the contribution of viscoelasticity to the total strain development

\subsection{Conclusion}

The fatigue life of angle-ply Glass/epoxy and Graphite/epoxy laminates was calculated through a Simplified Unit Cell Micromechanical approach including the effect of damage and viscoelasticity at two different temperatures. The agreement between experiment and prediction appears to be reasonable. The contributions made by viscoelasticity and cycling to material softening were individually investigated. Effect of viscoelasticity was taken into account based on the assumption that the fatigue mean stress acts like constant load which causes creep in the material. It was observed that the share of viscoelasticity is more significant throughout most of the life of the specimen. However, towards the end of the life, stiffness degradation plays a more dominant role in the evolution of damage. It was also found that in spite of the increase in creep strain at higher temperatures; its contribution to strain development during fatigue loading is compromised by the shorter life of the specimen when compared to lower temperatures. Regarding the effect of mean stress, one could see that 
higher mean stress expedites the failure of the material mainly through cycling as compared to viscoelasticity. 


\section{CHAPTER 7: A CASE-STUDY ON THE CREEP AND FATIGUE OF HIGH TEMPERATURE RP-46 POLYIMIDE COMPOSITE}

The present study utilizes a micromechanical model based on the assumptions of Simplified Unit Cell Micromechanical (SUCM) model to study the viscoelastic response of AS4-12K /RP46 composites within a temperature range of 180 to $270^{\circ} \mathrm{C}$. It is assumed that within this short period of time, the effect of chemical aging can be neglected. Since in a micromechanical approach, the matrix and the fibers have to be modeled separately, the first part of this study is dedicated to the viscoelastic characterizing of RP46 polyimide resin to be used as the matrix phase of the composite material. In the present approach, upon the characterization of the matrix material, the obtained properties are input to the Simplified Unit Cell Micromechanical model to study the creep and fatigue behavior of the composite.

\subsection{RP46 Polyimide Resin}

RP46 is a high temperature polyimide composite resin created by NASA as an alternative for the existing polyimide systems. This high temperature resin has been specifically developed for aerospace applications. The operating temperature of RP46 ranges from -101 to $357^{\circ} \mathrm{C}$. Another quality of RP46 is its lower moisture absorption which makes it less vulnerable to moisture induced damage. RP46 also possesses excellent thermal oxidative stability and resistance to microcracking. 


\subsection{Viscoelastic Characterization of RP46 Neat Resin}

Figure 7.1 shows a sketch of the RP46 sample used for tensile and creep tests. The sample is cut by water jet from a $12.7 \mathrm{~cm}$ by $3.81 \mathrm{~cm}$ plate with a thickness of $0.254 \mathrm{~cm}$. The plate was manufactured by compression molding for 2 hours at 137.9 bar and $325^{\circ} \mathrm{C}$.

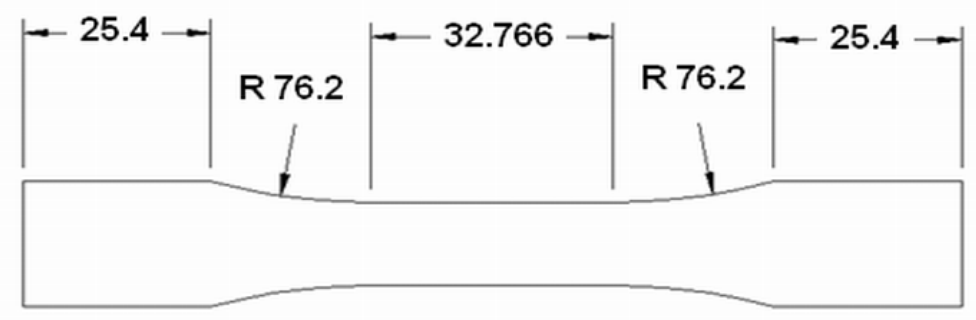

Figure 7-1: A sketch of the neat resin sample (All dimensions are in $\mathrm{mm}$ )

\subsubsection{Experimental setup}

Temperatures for the tests to characterize the viscoelastic behavior of RP46 were chosen to be 180,220 and $270^{\circ} \mathrm{C}$ to simulate service conditions which are typically below the material's glass transition temperatures. The static and creep tests were performed in accordance with the ASTM D3039M - 08 and D2990 - 09, respectively. The experiments were performed under uniaxial tension using a servo-hydraulic MTS machine equipped with a $100 \mathrm{kN}$ load cell. The testing machine was also equipped with a crosshead displacement sensor (LVDT). The samples were heated in a closed type cylindrical shell furnace, ATS model 3210. In order to maintain the temperature and prevent heat loss, insulation was put between the furnace and the grip. Figure 7.2 shows the furnace with a mounted sample inside it. 


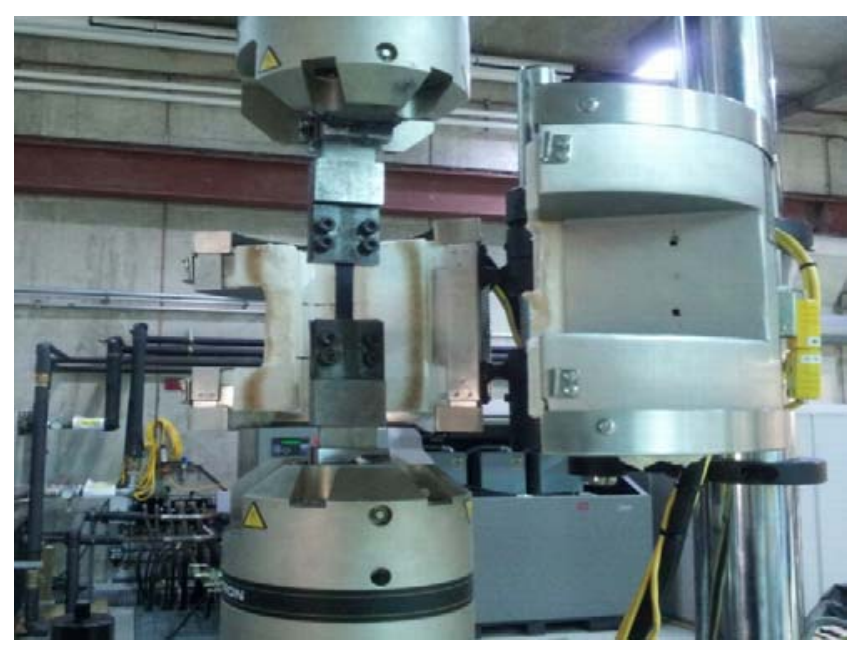

Figure 7-2: The experimental setup for creep tests

\subsubsection{Tension and creep tests}

Tension tests were performed at the aforementioned three temperatures 180,220 and $270{ }^{\circ} \mathrm{C}$ at a loading rate of $2 \mathrm{~mm} / \mathrm{min}$. The peak stress measured in the tensile tests was considered to be the ultimate strength (UTS) of the material at that very temperature. Both the modulus and ultimate strength were seen to decrease with temperature indicating that the epoxy becomes more ductile as we get closer to $T_{g}$. Once the ultimate strength of the material was determined, creep tests were conducted at $20 \%$ of the UTS for each temperature to ensure linear viscoelasticity. After loading the sample to the target stress, it was allowed to creep for 30 minutes. The results from the creep test for different temperatures are shown in figure 7.3. It is noted that the instantaneous compliance of the material is increasing with increasing temperature. 


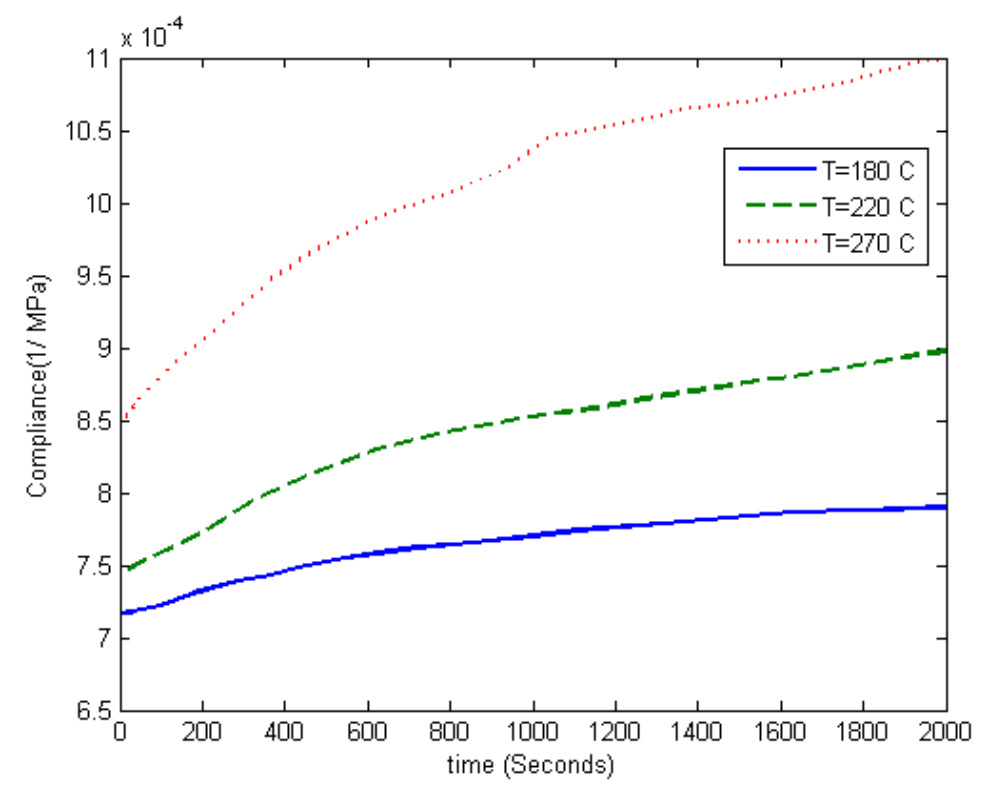

Figure 7-3: Creep compliance data for $20 \%$ UTS for different temperatures

\subsubsection{Prony Series representation of the creep data}

The obtained creep data is used to determine the Prony series for RP46 through the use of the Time-Temperature Superposition Principle (TTSP) assuming that material behavior is linearly viscoelastic at $20 \%$ of the UTS. According to TTSP, the viscoelastic behavior at one temperature can be related to that of another temperature by a change in time scale. The Prony series representation of the compliance is:

$D(t)=D_{0}+\sum_{i=1}^{N} D_{i}\left(1-\exp \left(-\lambda_{i} t\right)\right)$

Where $D(t)$ is the compliance at time t, and $D_{0}$ is the elastic compliance. $D_{i}$ and $\lambda_{i}$ are the magnitudes of compliance and reciprocal of the retardation times, respectively which correspond to the ith Kelvin-Voigt element and $N$ is the total number of elements to represent material behavior. In order to create the master curve, the compliance data at $220^{\circ} \mathrm{C}$ and 270 ${ }^{\circ} \mathrm{C}$ is shifted horizontally on a log scale to the reference temperature of $180^{\circ} \mathrm{C}$ so as to form a 
continuous curve. Figure 7.4 shows the master curve obtained through this procedure along with the value of the horizontal shift factor. It can be seen that the master curve can provide creep compliance predictions up to 88 hours at the reference temperature. The next step is to fit the Prony series representation of the compliance to the obtained master curve. The problem of curve fitting of Equation 6.1 is one of a nonlinear optimization. The master curve in figure 7.5 has been fitted to a 5 term Prony series using commercial curve fitting software, Origin 8.5. As shown in figure 7.5, a reasonable fit has been obtained to the experimentally generated master curve. The Prony series coefficients are listed in Table 7.1.

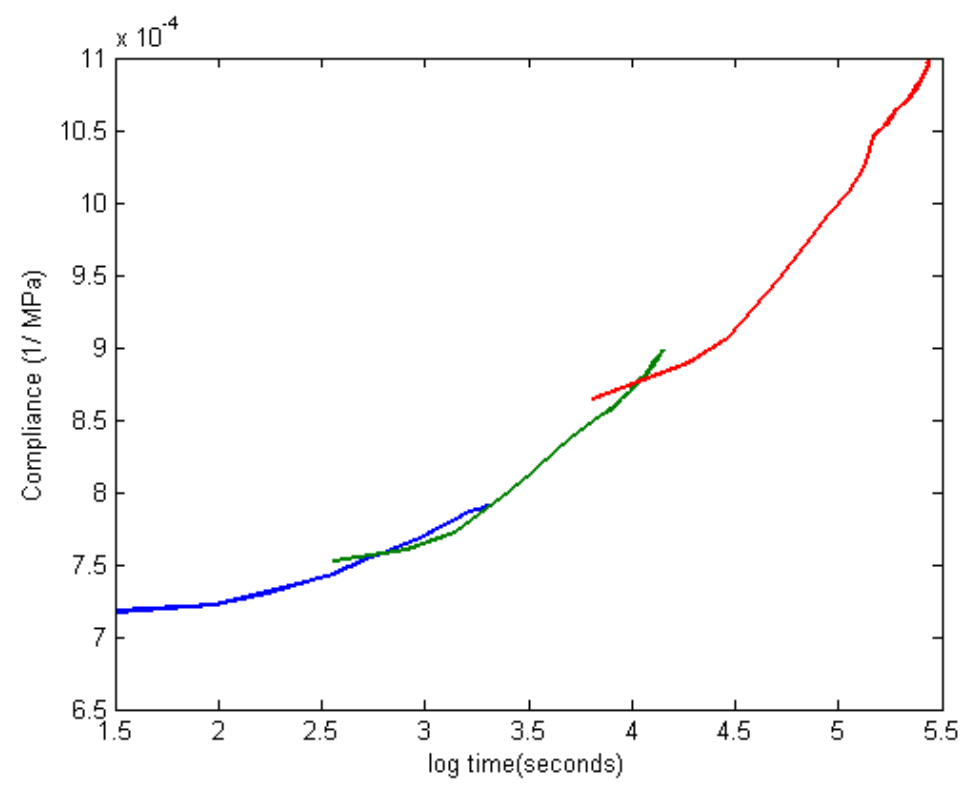

Figure 7-4: Creep compliance versus shifted time (Master curve) 


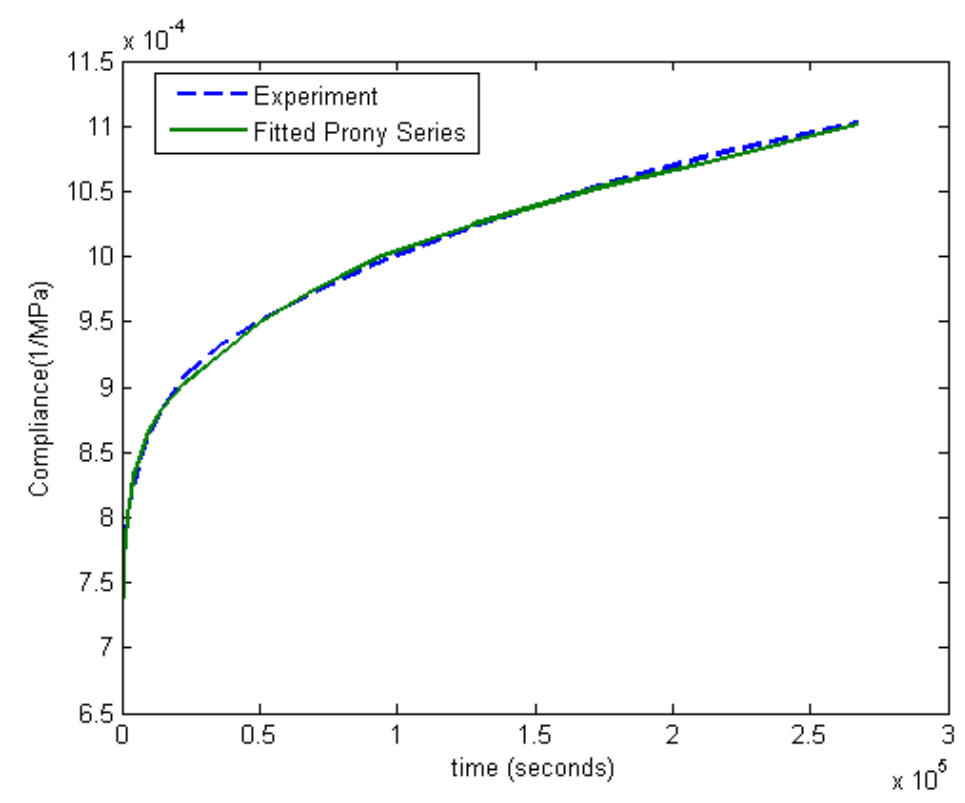

Figure 7-5: Prony Series fit to the master curve

Table 7-1: Prony Series coefficients for RP46 resin

\begin{tabular}{|c|c|c|}
\hline$n$ & $\lambda_{n}$ & $D_{n}\left(\mathrm{MPa}^{-1}\right)$ \\
\hline 0 & $7.1642 \mathrm{e}-004$ & - \\
\hline 1 & $3.5944 \mathrm{e}-008$ & $5 \mathrm{e}-3$ \\
\hline 2 & $6.7203 \mathrm{e}-005$ & $1 \mathrm{e}-3$ \\
\hline 3 & $1.0214 \mathrm{e}-004$ & $1 \mathrm{e}-4$ \\
\hline 4 & $7.7623 \mathrm{e}-005$ & $1 \mathrm{e}-5$ \\
\hline 5 & $2.9767 \mathrm{e}-004$ & $4 \mathrm{e}-5$ \\
\hline
\end{tabular}

\subsection{Composite Laminate Creep Response}

The composite Laminates were prepared by the hand lay-up technique using 8 layers of AS4$12 \mathrm{~K}$ unidirectional fiber and the RP46 resin with 0 degree orientation. Specimen dimensions were $200 \mathrm{~mm} \times 20 \mathrm{~mm} \times 3 \mathrm{~mm}$. A scanning electron microscope (JEOL, JSM-6380) was used to calculate the volume fraction of the constituents. The average volume fraction of fiber was found to be approximately $37 \%$. The same curing cycle as the one for neat resin was also used for the composite. The composite specimens were first subjected to static tension tests at 180 and $270^{\circ} \mathrm{C}$ in the transverse direction in order to determine the ultimate tensile strength. 
Next, the samples were subjected to 30 minute creep tests at $30 \%$ and $60 \%$ of the UTS for each temperature. The vicoelastic properties of the neat resin obtained in the previous section (Table 7.1) were incorporated into the Simplified Unit Cell Micromechanical (SUCM) model to predict the creep response of the RP46 resin based composite system. Based on the neat resin characterization, since $180^{\circ} \mathrm{C}$ is the reference temperature, $f_{1}(T), f_{2}(T)$ and $a_{T}(T)$ (Equation 4.5) are all equal to unity. When the creep load is $30 \%$ of the UTS, the material has been considered to be linearly viscoelastic i.e. $g_{0}(\sigma)=g_{1}(\sigma)=g_{2}(\sigma)=a(\sigma)=1$. Figure 7.6 shows the creep response of the composite material at $180^{\circ} \mathrm{C}$ for $30 \%$ of the UTS compared to the experimental results. At the creep load of $60 \%$ of the UTS, the material behavior becomes nonlinear viscoelastic. In order to include the nonlinearity of the material, only $g_{2}(\sigma)$ and $a(\sigma)$ are taken to be equal to 1 . Now, assuming that plastic strain is negligible, $g_{0}(\sigma)$ can be found as the ratio of the instantaneous creep response to that of linear elastic material i.e. at $30 \%$ of the UTS, while $g_{1}(\sigma)$ is obtained by fitting Schapery constitutive Equation to the experimental creep curve. Figure 7.7 compares the creep response of the material at $180^{\circ} \mathrm{C}$ for $60 \%$ of the UTS with the experimental data before and after including nonlinearity. It can be seen that increasing the creep load gives rise to the softening of the material and consequently the creep strain. 


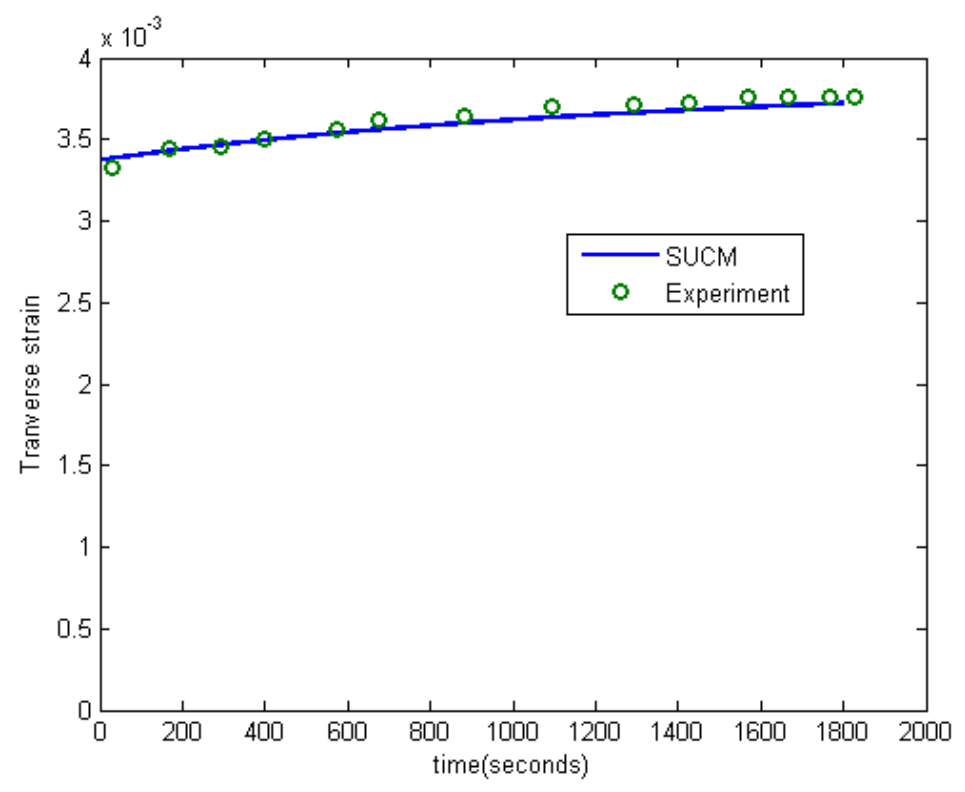

Figure 7-6: Creep response of the composite at $180^{\circ} \mathrm{C}$ for $30 \%$ of the UTS

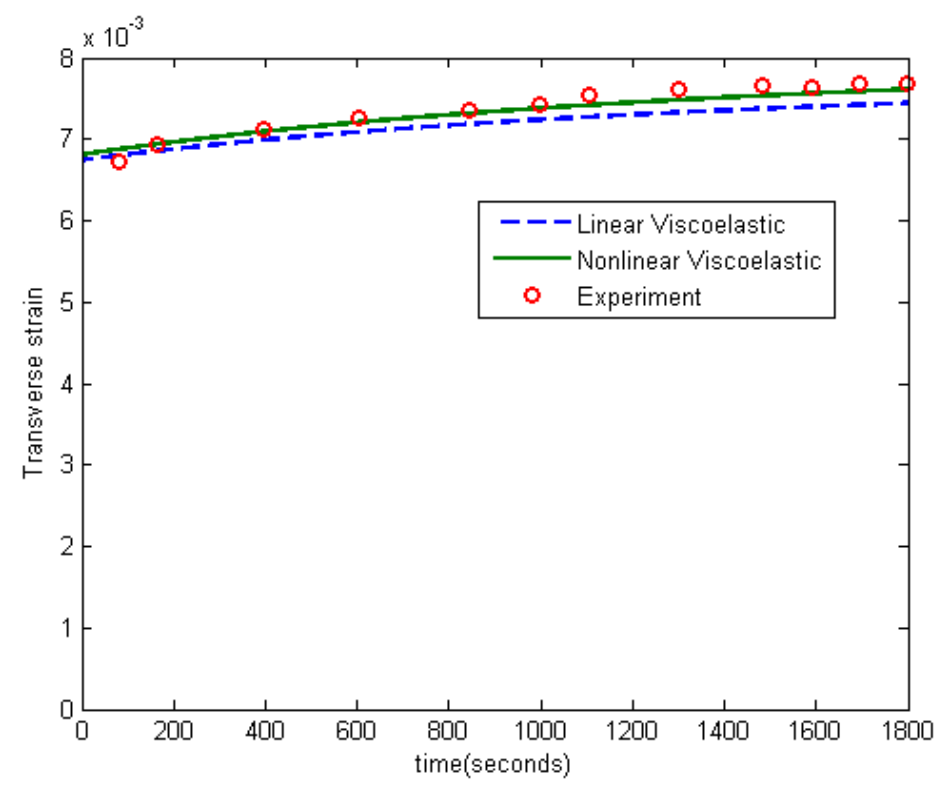

Figure 7-7: Creep response of the composite at $180^{\circ} \mathrm{C}$ for $60 \%$ of the UTS with the experimental data before and after including nonlinearity

The creep response of the composite material at $270{ }^{\circ} \mathrm{C}$ for $30 \%$ of the UTS in comparison with the experiment is depicted in Figure 7.8. The effect of temperature has been taken into account through the shift factor $a_{T}(T)$ as well as $f_{1}(T)$. The temperature increase is seen to have caused the acceleration of the creep strain and softening of both the initial and time- 
dependent portions of the composite response. For $60 \%$ of the UTS at $270{ }^{\circ} \mathrm{C}, g_{0}(\sigma)$ and $g_{1}(\sigma)$ are obtained in a similar fashion as for the previous temperature. Figure 7.9 shows the creep response both before and after including nonlinearity. A closer look at Figures 7.7 and 7.9 shows that the effect of nonlinearity is more pronounced at $\mathrm{T}=270^{\circ} \mathrm{C}$.

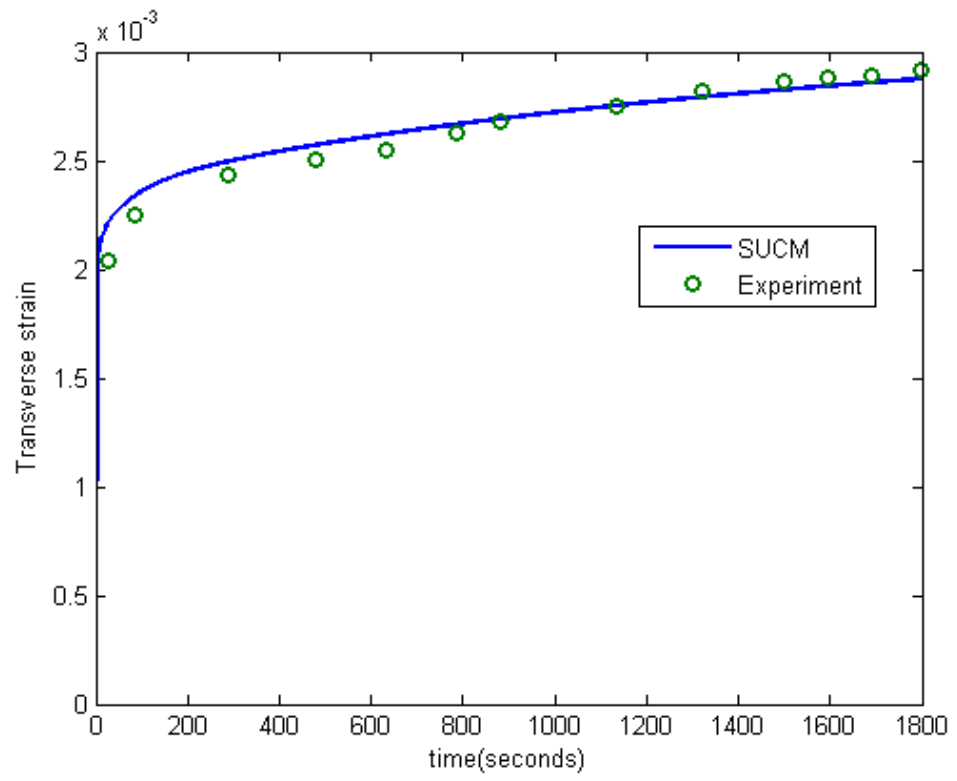

Figure 7-8: Creep response of the composite at $270{ }^{\circ} \mathrm{C}$ for $30 \%$ of the UTS 


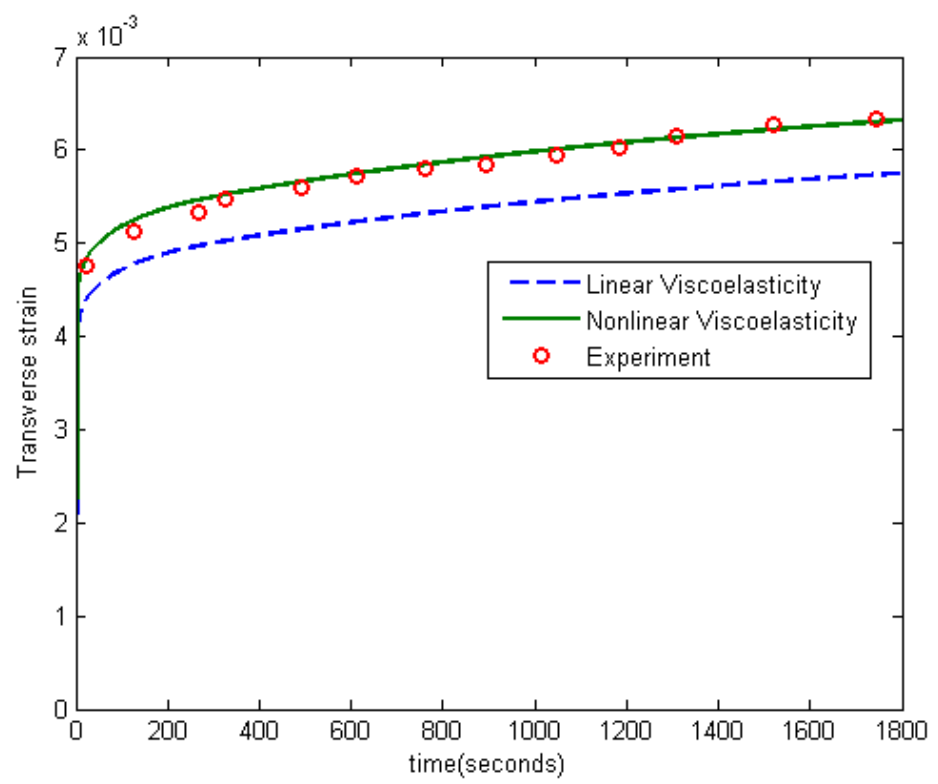

Figure 7-9: Creep response of the composite at $270{ }^{\circ} \mathrm{C}$ for $60 \%$ of the UTS with the experimental data before and after including nonlinearity

\subsection{Effect of Viscoelasticity on the fatigue of Composite Laminate}

The fatigue tests on AS4-12K /RP46 composites were conducted at 180 and $270{ }^{\circ} \mathrm{C}$ in the same experimental set-up shown in figure 7.2. The frequency of the tests was 5 cps and the stress ratio was fixed to be 0.15 . Figures 7.10 and 7.11 show the mean strain evolution during the fatigue loading of the composite at $180{ }^{\circ} \mathrm{C}$ for $40 \%$ and $60 \%$ of the ultimate strength, respectively. Similar to the observation in the previous chapter, it can be seen for the lower stress level that the contribution of viscoelasticity to the strain development is more. As shown in figure 7.10 , the strain evolution during the first $80 \%$ of the life of the specimen is dominated by creep. This is compared to the $65 \%$ of the life for the higher stress level in figure 7.11. Figure 7.12 show the mean strain evolution at $270{ }^{\circ} \mathrm{C}$ for $40 \%$ of the UTS. A comparison between figure 7.12 and 7.10 show that although increasing the temperature accelerates the evolution of viscoelastic strain, the role viscoelasticity is less prominent. In other words, after 
only a small portion of the total life, total strain evolution will be mainly due to the cyclic effect of fatigue loading.

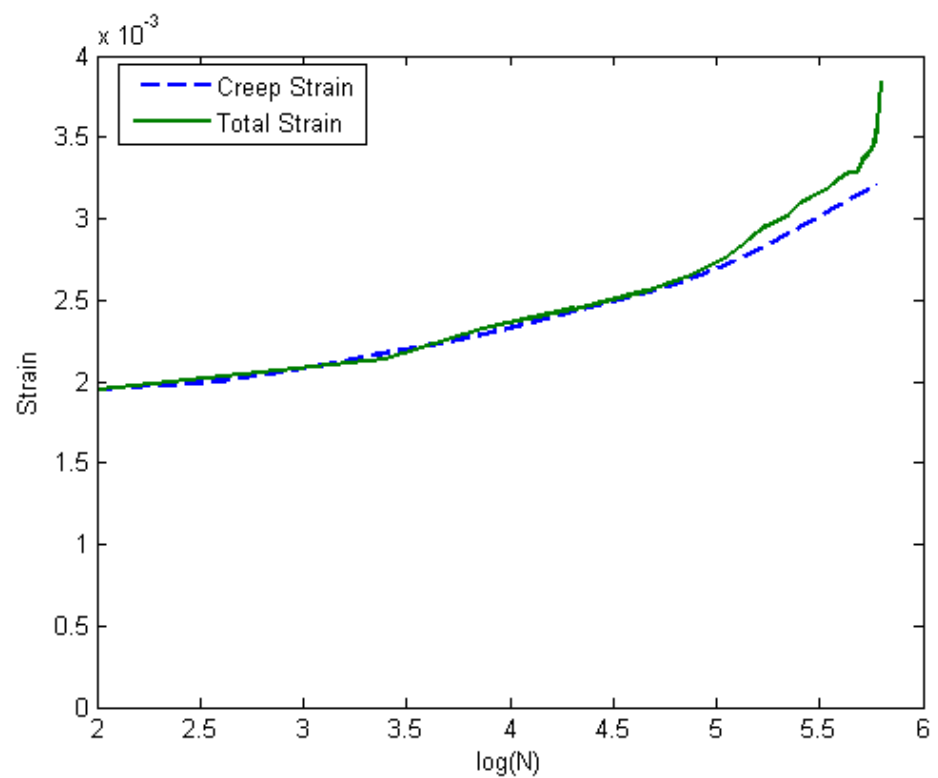

Figure 7-10: The mean strain evolution during the fatigue loading of the composite at $180{ }^{\circ} \mathrm{C}$ for $40 \%$ UTS

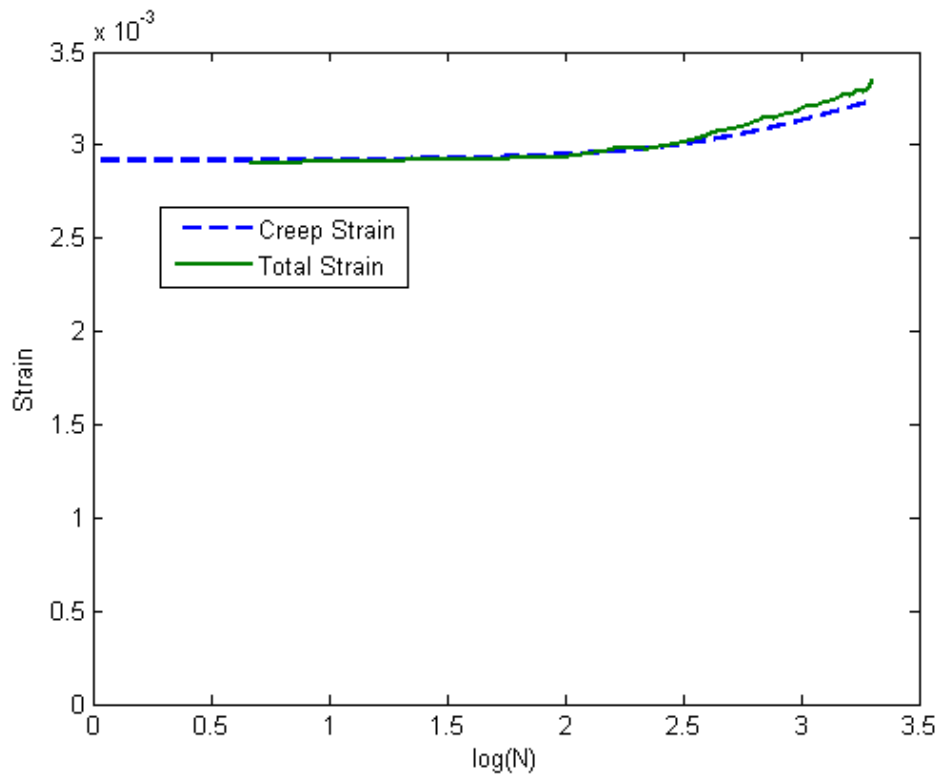

Figure 7-11: The mean strain evolution during the fatigue loading of the composite at $180{ }^{\circ} \mathrm{C}$ for $60 \%$ UTS 


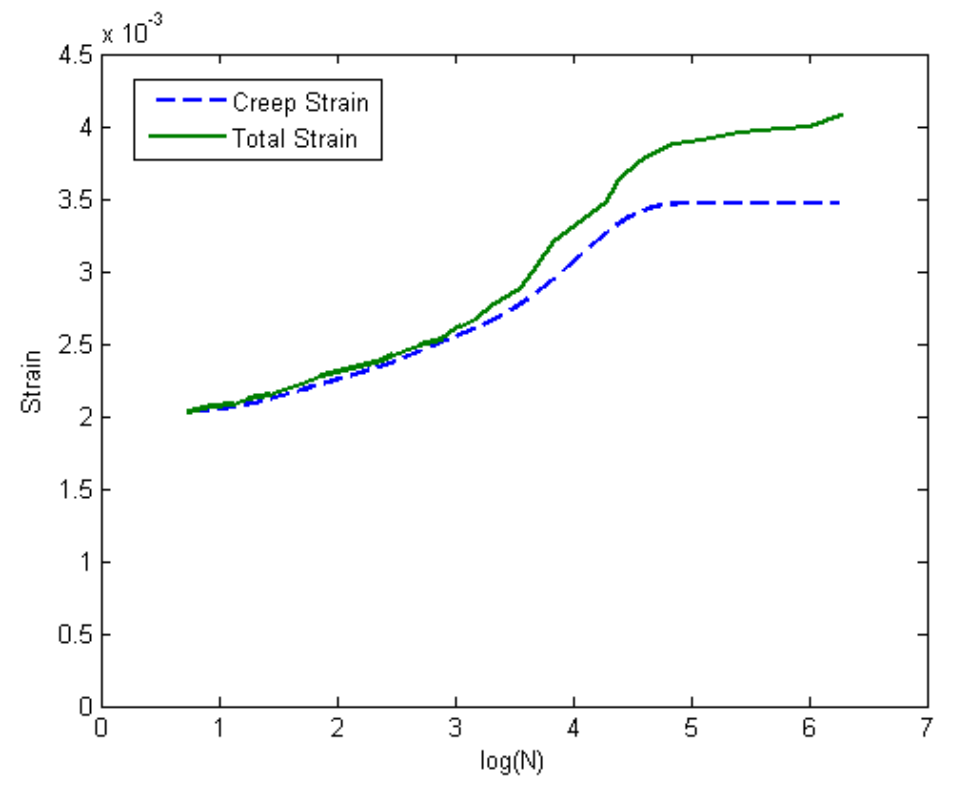

Figure 7-12: The mean strain evolution during the fatigue loading of the composite at $270{ }^{\circ} \mathrm{C}$ for $40 \%$ UTS

\subsection{Conclusion}

Creep tests were carried out on RP46 Polyimide resin at three different temperatures 180, 220 and $270^{\circ} \mathrm{C}$. The instantaneous compliance of the material was seen to be increasing with increasing temperature. A creep master curve was created by horizontally shifting the compliance data to the reference temperature of $180^{\circ} \mathrm{C}$ vs. $\log$ (time). While the duration of each creep test was 30 minutes, it can be seen that the master curve can provide creep compliance predictions for up to 88 hours at the reference temperature. A 5-term Prony series was fit to the master curve. The acquired viscoelatic properties were then incorporated into a Simplified Unit Cell Micromechanical model to study the creep response of the RP46 resin based composite system. The creep tests on the AS4-12K /RP46 composite revealed two types of nonlinearities, one due to stress and the other one due to temperature. Both these nonlinearities can be modeled through the use of proper coefficients in the constitutive equation of the matrix material. The temperature increase was seen to have caused the 
acceleration of the creep strain and softening of both the initial and time-dependent portions of the composite response. It was also observed that higher temperature gives rise to stress nonlinearities. At higher the temperature, despite the increase in the viscoelastic strain, the role of viscoelastity is seen to have decreased compared to the lower temperature. 


\section{CHAPTER 8: CONCLUSIONS}

\subsection{Conclusions}

The most notable conclusions reached in the present thesis are as follows:

- The assumption of thermorheologically simple behavior might not capture the true behavior of some polymer matrix materials.

- The stress-strain response of the composite material under a monotonically increasing stress was seen to be affected by temperature in transverse and shear loadings which were dominated by matrix properties. When subject to creep loading, the acceleration of the creep strain and softening of the initial and time-dependent portions of the composite response with increasing temperature was noticeable. Under cyclic creep loading, the total strain was seen to increase due to the accumulation of creep strain during each cycle although the rate of this increase drops from cycle to cycle as the number of the cycles increases.

- It was observed that throughout the fatigue life a polymer matrix composite material, the share of viscoelasticity was more significant throughout most of the life of the specimen. However, towards the end of the life, stiffness degradation played a more dominant role in the evolution of damage. It was also found out that in spite of the increase in creep strain at higher temperatures, its contribution to strain development during fatigue loading was compromised by the shorter life of the specimen when compared to lower temperatures. Regarding the effect of mean stress, one could see that higher mean stress expedites the failure of the material mainly through cycling as compared to viscoelasticity. 


\subsection{Contributions}

This research work has so far led to the publication and submission of the following Journal

Papers:

1. A. Sayyidmousavi, R. Falahtgar, Z. Fawaz and H. Bougherara, "A generalized three dimensional energy criterion for the creep failure of high temperature polymer matrix composites using a micromechanical approach", Journal of Reinforced Plastics and Composites. 2014; 33(4): 380-388.

2. A. Sayyidmousavi, R. Falahtgar, Z. Fawaz and H. Bougherara, "Thermo-mechanical Viscoelastic Response of a Unidirectional Graphite/Polyimide Composite at Elevated Temperatures Using a Micromechanical Approach", Journal of Composite Materials. Published online February 6, 2014. doi:10.1177/0021998314521255

3. A. Sayyidmousavi, H. Bougherara, I. ElSawi and Z. Fawaz "A Micromechanical approach for the prediction of the time-dependent failure of high temperature polymer matrix composites" Mechanics of Time-dependent Materials. 2014; 18(2): 423-436

4. A. Sayyidmousavi, H. Bougherara and Z. Fawaz "A micromechanical approach for the fatigue failure prediction of unidirectional polymer matrix composites in off-axis loading including the effect of viscoelasticity", Submitted to the Journal of Advanced Composite Materials. May 06, 2014. Accepted for publication July 01, 2014.

5. A. Sayyidmousavi, H. Bougherara and Z. Fawaz "The role of viscoelasticity on the fatigue of angle-ply polymer matrix composites at high and room temperatures- $A$ micromechanical approach", Submitted to the Journal of Applied Composite Materials. March 15, 2014. Accepted for publication July 06, 2014.

6. A. Sayyidmousavi, H. Bougherara, I. ElSawi and Z. Fawaz "Investigation of the Viscoelastic Response of High Temperature AS4-12K /RP46 Composites Using a Micromechanical Approach", Submitted to the Journal of Polymer Composites. March 12, 2014. Awaiting referees' decision. 


\subsection{Suggestions for future work}

As mentioned previously, the present research attempted to fill the gap in the literature by studying the effect of temperature on the elastic and viscoelastic response of polymer matrix composites, and proposing a methodology to investigate the role of viscoelasticity on the fatigue failure of polymer matrix composite materials at room and high temperatures through a micromechanical approach. What has been done in this thesis and the methodology presented is in fact, a stepping stone towards the application of high temperature polymer matrix composites in different fields, especially the aerospace industry. In particular, the following areas of research need to be explored:

- The present thesis introduces a micromechanical approach to study creep and the effect of viscoelasticity on the fatigue life of polymer matrix composites. This approach does have the potential to be applied to different composite materials. In particular, the effect of different parameters such as stress ratio and frequency on fatigue of high temperature polymer matrix materials including viscoelasticity can be investigated using the present approach.

- In the present work, the fiber constituent of the composite was assumed to be elastic and temperature independent. One potential area of research is to study composite materials with flax fibers in which the fiber has vicoelastic behavior, too

- The present work focused on thin composite laminates in which delamination is not a significant mode of failure. Studying the failure of thick composite laminates in which delamination is a dominant mode of damage can be another area of future work. 
- The micromechanical model used in this study is simplified one in which the RVE consists of only four subcells. Expanding the RVE to include more than 4 subcells and how it might improve the accuracy of the results would also be valuable. 


\section{REFERENCES}

Aboudi, J.:"A continuum theory for fiber-reinforced elastic-viscoplastic composites". International Journal of Engineering Science, 20(5): 605-621 (1982)

Aboudi, J.:"Micromechanical analysis of the strength of unidirectional fiber composites". Composites Science and Technology, 33(2): 79-96 (1988)

Agarwal, B. D., Broutman, L. J. and Chandrashekhara, K.:Analysis and Performance of Fiber Composites. John Wiley \& Sons (2006)

Aghdam, M. M. and Dezhsetan, A.:"Micromechanics based analysis of randomly distributed fiber reinforced composites using simplified unit cell model". Composite Structures, 71(3-4): 327-332 (2005)

Aghdam, M. M., Smith, D. J. and Pavier, M. J.:"Finite element micromechanical modelling of yield and collapse behaviour of metal matrix composites". Journal of the Mechanics and Physics of Solids, 48(3): 499-528 (2000)

Barnard, P. M., Butler, R. J. and Curtis, P. T.:"Fatigue Scatter of UD Glass Epoxy, a Fact or Fiction?". In: Composite Structures 3, Edited by I. H. Marshall. Springer Netherlands, 69-82 (1985)

Beaumont, P. W. R.:"The mechanics of fatigue damage in structural composite materials". Proceedings of 4th European Conference on Composite Materials (ECCM/4), Stuttgart, Germany (1990)

Bergmann, H. W. and Prinz, R.:"Fatigue life estimation of graphite/epoxy laminates under consideration of delamination growth". International Journal for Numerical Methods in Engineering, 27(2): 323-341 (1989)

Bhargava, P.:Temperature properties of HFPE-II-52 polyimide resin and composites. PhD Thesis. Cornell University (2007)

Bond, I. P.:"Fatigue life prediction for GRP subjected to variable amplitude loading". Composites Part A: Applied Science and Manufacturing, 30(8): 961-970 (1999)

Branco, C. M., Eichler, K. and Ferreira, J. M.:"Fatigue behavior of E-glass fiber reinforced phenolic composites: effect of temperature, mean stress and fiber surface treatment". Theoretical and Applied Fracture Mechanics, 20(2): 75-84 (1994)

Brinson, H. F.:"Matrix dominated time dependent failure predictions in polymer matrix composites". Composite Structures, 47(1-4): 445-456 (1999)

Brinson, H. F. and Brinson, L. C.:Polymer Engineering Science and Viscoelasticity. An Introduction. Springer (2008)

Brinson, H. F., Griffith, W. I. and Morris, D. H.:"Creep rupture of polymer-matrix composites". Experimental Mechanics, 21(9): 329-335 (1981) 
Broendsted, P., Andersen, S. I. and Lilholt, H.:"Fatigue damage accumulation and lifetime prediction of GFRP materials under block loading and stochastic loading". Risoe international symposium on materials science, Roskilde, Denmark (1997a)

Broendsted, P., Andersen, S. I. and Lilholt, H.:"Fatigue damage prediction by measurements of the stiffness degradation in polymer matrix composites". International Conference on fatigue of composites., France, Paris (1997b)

Canga, M. E., Becker, E. B. and Özüpek, Ş.:"Constitutive modeling of viscoelastic materials with damage - computational aspects". Computer Methods in Applied Mechanics and Engineering, 190(15-17):

2207-2226 (2001)

Caprino, G. and D'Amore, A.:"Flexural fatigue behaviour of random continuous-fibre-reinforced thermoplastic composites". Composites Science and Technology, 58(6): 957-965 (1998)

Caron, J. F. and Ehrlacher, A.:"Modeling of fatigue microcracking kinetics in crossply and experimental validation.". Proceedings of the International Conference on Fatigue of Composites, Paris, France (1997)

Carswell, W. S.:"Damage mechanics and composite behaviour". Composite Structures, 10(4): 335-342 (1988)

Case, S. W., Plunkett, R. B. and Reifsnider, K. L.:"Simulation methods for life and remaing strength of high temperature polymeric composites subjected to cyclic loads". In: High Temperature and Environmental Effects on Polymeric Composites, Edited by T. S. Gates and A. H. Zureick. ASTM STP 1302, 35-49 (1997)

Castelli, M. G., Sutter, J. K. and Benson, D.:Thermomechanical fatigue durability of T650-35/PMR-15 sheet molding compound. NASA Technical Report. NASA-TM-28806 (1998)

Christensen, R. M.:"Lifetime predictions for polymers and composites under constant load". Journal of Rheology (1978-present), 25(5): 517-528 (1981)

Christensen, R. M.:"An Evaluation of Linear Cumulative Damage (Miner's Law) Using Kinetic Crack Growth Theory". Mechanics of Time-Dependent Materials, 6(4): 363-377 (2002)

Crochet, M. J.:"Symmetric Deformations of Viscoelastic-Plastic Cylinders". Journal of Applied Mechanics, 33(2): 327-334 (1966)

Daniel, I. M. and Charewicz, A.:"Fatigue damage mechanisms and residual properties of graphite/epoxy laminates". Engineering Fracture Mechanics, 25(5-6): 793-808 (1986)

Degrieck, J. and Van Paepegem, W.:"Fatigue damage modeling of fibre-reinforced composite materials: Review". Applied Mechanics Reviews, 54(4): 279-300 (2001)

Dillard, D. A.:Creep and creep rupture of laminated graphite/epoxy composites. PhD Thesis. Virginia Polytechnic Institute and State University (1981) 
Ellyin, F. and El-Kadi, H.:"A fatigue failure criterion for fiber reinforced composite laminae". Composite Structures, 15(1): 61-74 (1990)

Epaarachchi, J. A. and Clausen, P. D.:"A new approach to a fatigue damage model for glass-fibre reinforced plastic composites". Proceedings of 7th International Conference on Composites Engineering (ICCE/7), Denver ,USA (2000)

Falahatgar, S., Salehi, M. and Aghdam, M.:"Nonlinear viscoelastic response of unidirectional fiberreinforced composites in off-axis loading". Journal of Reinforced Plastics and Composites, 28(15): 17931811 (2009)

Falahatgar, S., Salehi, M. and Aghdam, M. M.:"Micro-Macro Analysis of Viscoelastic Unidirectional Laminated Composite Plates Using DR Method". Applied Composite Materials, 17(5): 427-440 (2010)

Farrow, I. R.:Damage Accumulation and Degradation of Composite Laminates Under Aircraft Service Loading: Assessment and Prediction. PhD Thesis. Cranfield Institute of Technology (1989)

Fawaz, Z. and Ellyin, F.:"Fatigue failure model for fibre-reinforced materials under general loading conditions". Journal of Composite Materials, 28(15): 1432-1451 (1994)

Feng, X., Gilchrist, M. D., Kinloch, A. J. and Matthews, F. L.:"Development of a method for predicting the fatigue life of CFRP components". Proceedings of the International Conference on Fatigue of Composites, Paris, France (1997)

Findley, W. N., Lai, J. S. and Onaran, K.:Creep and Relaxation of Nonlinear Viscoelastic Materials. Dover Civil and Mechanical Engineering (1976)

Gregory, J. R. and Spearing, S. M.:"Constituent and composite quasi-static and fatigue fracture experiments". Composites Part A: Applied Science and Manufacturing, 36(5): 665-674 (2005)

Guedes, R. M.:"Durability of polymer matrix composites: Viscoelastic effect on static and fatigue loading". Composites Science and Technology, 67(11-12): 2574-2583 (2007)

Gyekenyesi, A. L., Gastelli, M. G., Ellis, J. R. and Burke, C. S.:A study of elevated temperature testing techniques for the fatigue behavior of PMCS: Application to T650-35/AMB21. NASA Technical Report. NASA-TM-106927 (1995)

Ha, K. and Schapery, R. A.:"A three-dimensional viscoelastic constitutive model for particulate composites with growing damage and its experimental validation". International Journal of Solids and Structures, 35(26-27): 3497-3517 (1998)

Haj-Ali, R. M. and Muliana, A. H.:"A multi-scale constitutive formulation for the nonlinear viscoelastic analysis of laminated composite materials and structures". International Journal of Solids and Structures, 41(13): 3461-3490 (2004a)

Haj-Ali, R. M. and Muliana, A. H.:"Numerical finite element formulation of the Schapery non-linear viscoelastic material model". International Journal for Numerical Methods in Engineering, 59(1): 25-45 (2004b) 
Halpin, J. C., Jerina, K. L. and Johnson, T. A.:"Characterization of composites for the purpose of reliability evaluation". In: Analysis of the test methods for high modulus fibers and composites Edited by ASTM STP 521, 5-64 (1973)

Harper, B. D. and Weitsman, Y.:"Characterization Method for a Class of Thermorheologically Complex Materials". Journal of Rheology, 29(1): 49-66 (1985)

Hashin, Z., Humphreys, E. A. and Goering, J.:"Analysis of thermoviscoelastic behavior of unidirectional fiber composites". Composites Science and Technology, 29(2): 103-131 (1987)

Hashin, Z. and Rotem, A.:"A fatigue failure criterion for fiber reinforced materials". Journal of Composite Materials, 7(4): 448-464 (1973)

Hawk, J.:"The Boeing 787 Dreamliner- More than an Airplane". Proceedings of the AIAA/AAAF Aircraft Noise and Emissions Reduction Symposium, Monterey, USA (2005)

Henaff-Gardin, C., Lafarie-Frenot, M. C. and Goupillaud, I.:"The use of a characteristic damage variable in the study of transverse cracking development under fatigue loading in cross-ply laminates".

Proceedings of the 2nd International Conference on Fatigue of Composites., Williamsburg, USA (2000)

Hiel, C.:The Nonlinear Viscoelastic Response of Resin Matrix Composites. PhD Thesis. Free University of Brussels (1983)

Highsmith, A. L. and Reifsnider, K. L.:"Stiffness-reduction mechanisms in composite laminates". In: Damage in composite materials, Edited by K. L. Reifsnider. ASTM STP 775, 103-117 (1982)

Hwang, W. and Han, K.:"Cumulative damage models and multi-stress fatigue life prediction". Journal of Composite Materials, 20(2): 125-153 (1986a)

Hwang, W. and Han, K. S.:"Fatigue of composites-fatigue modulus concept and life prediction". Journal of Composite Materials, 20(2): 154-165 (1986b)

Jen, M.-H. R., Tseng, Y.-C. and Lin, W.-H.:"Thermo-mechanical fatigue of centrally notched and unnotched AS-4/PEEK APC-2 composite laminates". International Journal of Fatigue, 28(8): 901-909 (2006)

Jen, M. H. R. and Lee, C. H.:"Strength and life in thermoplastic composite laminates under static and fatigue loads. Part I: Experimental". International Journal of Fatigue, 20(9): 605-615 (1998a)

Jen, M. H. R. and Lee, C. H.:"Strength and life in thermoplastic composite laminates under static and fatigue loads. Part II: Formulation". International Journal of Fatigue, 20(9): 617-629 (1998b)

Kawai, M.:"Damage mechanics model for off-axis fatigue behavior of unidirectional carbon fiberreinforced composites at room and high temperatures". Proceedings of 12th International Conference on Composite Materials (ICCM-12), Paris, France (1999) 
Kawai, M., Yajima, S., Hachinohe, A. and Kawase, Y.:"High-temperature off-axis fatigue behaviour of unidirectional carbon-fibre-reinforced composites with different resin matrices". Composites Science and Technology, 61(9): 1285-1302 (2001)

Kibler, K. and Carter, H.:"Viscoelastic parameters of epoxy resin from thermomechanical and electrical conductivity measurements". In: Composite Materials: Testing and Design, Edited by S. W. TSa. ASTM STP 674, 282-288 (1978)

Knauss, W. G.:"Delayed failure - the Griffith problem for linearly viscoelastic materials". International Journal of Fracture Mechanics, 6(1): 7-20 (1970)

Lai, J. and Bakker, A.:"3-D schapery representation for non-linear viscoelasticity and finite element implementation". Computational Mechanics, 18(3): 182-191 (1996)

Lo, Y. J., Liu, C. H., Hwang, D. G., Chang, J. F., Chen, J. C., Chen, W. Y. and Hsu, S. E.:"High temperature behaviors of an innovative polymeric matrix composite". In: High Temperature and Environmental Effects on Polymeric Composites, Edited by C. E. Harris and T. S. Gates. ASTM STP 1174, 66-77 (1993)

Lou, Y. and Schapery, R. A.:"Viscoelastic characterization of a nonlinear fiber-reinforced plastic". Journal of Composite Materials, 5(2): 208-234 (1971)

Mahieux, C. A., Reifsnider, K. L. and Jackson, J. J.:"Property Modeling across Transition Temperatures in PMC's: Part III. Bending Fatigue". Applied Composite Materials, 8(4): 249-261 (2001)

Marais, C. and Villoutreix, G.:"Analysis and modeling of the creep behavior of the thermostable PMR15 polyimide". Journal of Applied Polymer Science, 69(10): 1983-1991 (1998)

Meador, M.:"Materials Challenge Diversification and the Future". Materials Challenge Diversification and the Future. Proceedings of the 40th International SAMPE Symposium and Exhibition(1995)

Meador, M. A. B.:Addition polymers from 1, 4, 5, 8-Tetrahydro- 1, 4; 5, 8-diepoxyanthracene and Bisdienes: Processable resins for high temperature applications. NASA Technical Report. NASA-TM-89839 (1987)

Miyano, Y., McMurray, M. K., Kitade, N., Nakada, M. and Mohri, M.:"Role of matrix resin on the flexural static behavior of unidirectional pitch-based carbon fiber laminates". Advanced Composite Materials, 4(2): 87-99 (1994)

Miyano, Y., Nakada, M., Kudoh, H. and Muki, R.:"Prediction of tensile fatigue life for unidirectional CFRP". Journal of Composite Materials, 34(7): 538-550 (2000)

Miyano, Y., Nakada, M., McMurray, M. K. and Muki, R.:"Prediction of flexural fatigue strength of CRFP composites under arbitrary frequency, stress ratio and temperature". Journal of Composite Materials, 31(6): 619-638 (1997)

Miyano, Y., Nakada, M. and Sekine, N.:"Accelerated testing for long-term durability of FRP laminates for marine use". Journal of Composite Materials, 39(1): 5-20 (2005) 
Montesano, J., Fawaz, Z., Behdinan, K. and Poon, C.:Fatigue of Polymer Matrix Composites At elevated Temperatures. Nova Science Publishers, Inc. (2011)

Moura Branco, C., Ferreira, J. M., Fael, P. and Richardson, M. O. W.:"A comparative study of the fatigue behaviour of GRP hand lay-up and pultruded phenolic composites". International Journal of Fatigue, 18(4): 255-263 (1996)

Muliana, A. H. and Haj-Ali, R.:"A multi-scale framework for layered composites with thermorheologically complex behaviors". International Journal of Solids and Structures, 45(10): 2937-2963 (2008)

Muliana, A. H. and Sawant, S.:"Responses of viscoelastic polymer composites with temperature and time dependent constituents". Acta Mechanica, 204(3-4): 155-173 (2009)

Naghdi, P. M. and Murch, S. A.:"On the Mechanical Behavior of Viscoelastic/Plastic Solids". Journal of Applied Mechanics, 30(3): 321-328 (1963)

Odegard, G. and Kumosa, M.:"Elastic-plastic and failure properties of a unidirectional carbon/PMR-15 composite at room and elevated temperatures". Composites Science and Technology, 60(16): 29792988 (2000)

Ogin, S. L., Smith, P. A. and Beaumont, P. W. R.:"Matrix cracking and stiffness reduction during the fatigue of a (0/90)s GFRP laminate". Composites Science and Technology, 22(1): 23-31 (1985)

Ozupek, S. and Becker, E. B.:"Constitutive Modeling of High-Elongation Solid Propellants". Journal of Engineering Materials and Technology, 114(1): 111-115 (1992)

Ozupek, S. and Becker, E. B.:"Constitutive Equations for Solid Propellants". Journal of Engineering Materials and Technology, 119(2): 125-132 (1997)

Pochiraju, K. V., Tandon, G. P. and Schoeppner, G. A.:"Evolution of stress and deformations in hightemperature polymer matrix composites during thermo-oxidative aging". Mechanics of TimeDependent Materials, 12(1): 45-68 (2008)

Ramakrishnan, V. and Jayaraman, N.:"Mechanistically based fatigue-damage evolution model for brittle matrix fibre-reinforced composites". Journal of Materials Science, 28(20): 5592-5602 (1993)

Reifsnider, K. L. and Gao, Z.:"A micromechanics model for composites under fatigue loading". International Journal of Fatigue, 13(2): 149-156 (1991)

Reifsnider, K. L. and Stinchomb, W. W.:"A Critical Element Model of the Residual Strength and Life of Fatigue Loaded Coupons". In: Composite Materials: Fatigue and Fracture, Edited by H. T. Hahn. ASTM STP 907, 298-303 ( 1986)

Reiner, M. and Weissenberg, K.:"A Thermodynamic Theory of the Strength of the Materials". Rheological Leaflet, 10(1): 12-20 (1939) 
Robertson, D. D. and Mall, S.:"Micromechanical relations for fiber-reinforced composites using the free transverse shear approach". Journal of composites technology \& research, 15(3): 181-192 (1993)

Robertson, D. D. and Mall, S.:"A non-linear micromechanics-based analysis of metal-matrix composite laminates". Composites Science and Technology, 52(3): 319-331 (1994)

Rotem, A. and Nelson, H. G.:A temperature-dependent fatigue failure criterion for graphite/epoxy laminates. NASA technical Memorandum. NASA-TM-78538 (1978)

Rupnowski, P., Gentz, M. and Kumosa, M.:"Mechanical response of a unidirectional graphite fiber/polyimide composite as a function of temperature". Composites Science and Technology, 66(78): 1045-1055 (2006)

Sacks, S. and Johnson, W. S.:"Effects of thermal aging on the mechanical behavior of K3B matrix material". Journal of Thermoplastic Composite Materials, 11(5): 429-442 (1998)

Sadkin, Y. and Aboudi, J.:"Viscoelastic behavior of thermo-rheologically complex resin matrix composites". Composites Science and Technology, 36(4): 351-365 (1989)

Schaff, J. R. and Davidson, B. D.:"Life prediction methodology for composite structures. Part IConstant amplitude and two-stress level fatigue". Journal of Composite Materials, 31(2): 128-157 (1997)

Schapery, R. A.:"On the characterization of nonlinear viscoelastic materials". Polymer Engineering \& Science, 9(4): 295-310 (1969)

Schapery, R. A.:"Theory of Crack Initiation and Growth in Viscoelastic Media. 1. Theoretical Development". International Journal of Fracture, 11(1): 141-159 (1975a)

Schapery, R. A.:"Theory of Crack Initiation and Growth in Viscoelastic Media. 2. Approximate Methods of Analysis". International Journal of Fracture, 11(3): 369-388 (1975b)

Schapery, R. A.:"Theory of Crack Initiation and Growth in Viscoelastic Media. 3. Analysis of Continuous Growth". International Journal of Fracture, 11(4): 549-562 (1975c)

Scott, D. W., Lai, J. S. and Zureick, A.-H.:"Creep behavior of fiber-reinforced polymeric composites: a review of the technical literature". Journal of Reinforced Plastics and Composites, 14(6): 588-617 (1995)

Sendeckyj, G. P.:"Life Prediction for Resin-Matrix Composite Materials". In: Composite Materials Series: Fatigue of Composites, Edited by K. L. Reifsnider. Elsevier Science Publishers, 431-483 (1990)

Serafini, T. T. and Vannucci, R. D.:Tailor making high performance graphite fiber reinforced PMR polyimides. NASA technical Report. NASA- LEW-12416 (1975)

Shah, I. R., Murthy, P. L. N. and Chamis, C. C.:Effect of Cyclic Thermo-Mechanical Loads on Fatigue Reliability in Polymer Matrix Composites. NASA Technical Report. NASA-TM-107091 (1995) 
Shimokawa, T., Kakuta, Y., Hamaguchi, Y. and Aiyama, T.:"Static and fatigue strengths of a G40$800 / 5260$ carbon fiber/bismaleimide composite material at room temperature and $150 \mathrm{C}$ ". Journal of Composite Materials, 42(7): 655-679 (2008)

Shimokawa, T., Kakuta, Y., Saeki, D. and Kogo, Y.:"Carbon plain-weave fabric low-temperature vacuum cure epoxy composite: static and fatigue strength at room and high temperatures and practicality evaluation". Journal of Composite Materials, 41(18): 2245-2265 (2007)

Shirazi, A. and Varvani-Farahani, A.:"A Stiffness Degradation Based Fatigue Damage Model for FRP Composites of (0/0) Laminate Systems". Applied Composite Materials, 17(2): 137-150 (2010)

Shokrieh, M. M. and Lessard, L. B.:"Progressive fatigue damage modeling of composite materials, Part I: Modeling". Journal of Composite Materials, 34(13): 1056-1080 (2000a)

Shokrieh, M. M. and Lessard, L. B.:"Progressive fatigue damage modeling of composite materials, Part II: Material characterization and model verification". Journal of Composite Materials, 34(13): 1081$1116(2000 b)$

Sjögren, A. and Asp, L. E.:"Effects of temperature on delamination growth in a carbon/epoxy composite under fatigue loading". International Journal of Fatigue, 24(2-4): 179-184 (2002)

Spearing, S. M. and Beaumont, P. W. R.:"Fatigue damage mechanics of composite materials. I: Experimental measurement of damage and post-fatigue properties". Composites Science and Technology, 44(2): 159-168 (1992a)

Spearing, S. M. and Beaumont, P. W. R.:"Fatigue damage mechanics of composite materials Part III: Prediction of post-fatigue strength". Composites Science and Technology, 44(4): 299-307 (1992b)

St. Clair, A. K. and St. Clair, T. L.:A review of high temperature adhesives. NASA Technical Report. NASATM-83141 (1981)

Thesken, J. C., Shin, E. E., Sutter, J. K., Burke, C. and Fink, J.:"Thermomechanical Fatigue of Polyimide Composites in Reusable Propulsions Systems". International SAMPE Technical Conference(2004)

Tiano, T., Hurley, W., Roylance, M., Landrau, N. and Kovar, R. F.:"Reactive Plasticizers for Resin Transfer-Molding of High Temperature PMR Composites". INternational Sample Technical Conference, Boston, MA (2000)

Tuttle, M. E. and Brinson, H. F.:"Prediction of the long-term creep compliance of general composite laminates". Experimental Mechanics, 26(1): 89-102 (1986)

Uematsu, Y., Kitamura, T. and Ohtani, R.:"Delamination behavior of a carbon-fiber-reinforced thermoplastic polymer at high temperatures". Composites Science and Technology, 53(3): 333-341 (1995)

Walrath, D. E.:"Viscoelastic response of a unidirectional composite containing two viscoelastic constituents". Experimental Mechanics, 31(2): 111-117 (1991) 
Whitworth, H. A.:"A stiffness degradation model for composite laminates under fatigue loading". Composite Structures, 40(2): 95-101 (1997)

Whitworth, H. A.:"Evaluation of the residual strength degradation in composite laminates under fatigue loading". Composite Structures, 48(4): 261-264 (2000)

Xie, W., Pan, W.-P. and Chuang, K. C.:"Thermal characterization of PMR polyimides". Thermochimica Acta, 367-368(0): 143-153 (2001)

Yang, J., Jones, D., Yang, S. and Meskini, A.:"A stiffness degradation model for graphite/epoxy laminates". Journal of Composite Materials, 24(7): 753-769 (1990)

Yen, S.-C. and Morris, D. H.:"Accelerated characterization of a chopped fiber composite using a strain energy failure criterion". Polymer Composites, 10(4): 249-255 (1989)

Zhurkov, S. N.:"Kinetic concept of the strength of solids". International Journal of Fracture, 26(4): 295307 (1984) 